\title{
Electrochemical Tandem Olefination and Hydrogenation Reaction with Ammonia
}

Xiaofeng Zhang, ${ }^{\text {a }}$ Runze Jiang, ${ }^{a}$ Xu Cheng,

a. Institute of Chemistry and Biomedical Sciences, Jiangsu Key Laboratory of Advanced Organic Materials, National Demonstration Center for Experimental Chemistry Education, School of Chemistry and Chemical Engineering, Nanjing University, Nanjing, 210023, China.

b. State Key Laboratory of Elemento-organic Chemistry, Nankai University, Tianjin, 300071, China.

c. State Key Laboratory Breeding Base of Green Chemistry-Synthesis Technology, Zhejiang University of Technology, Hangzhou, 310032, China.

E-mail: chengxu@nju.edu.cn 


\section{Table of Contents}

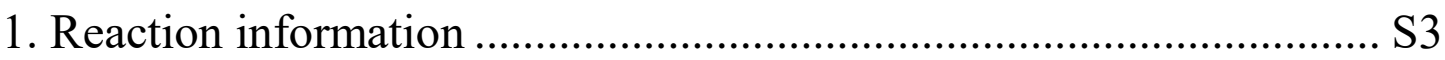

2. Investigation of reactants and intermediate ....................................... S6

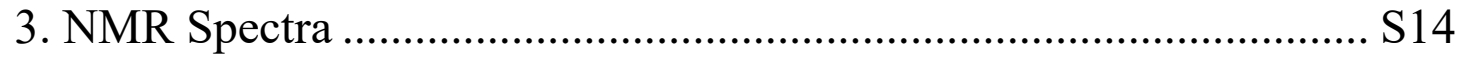




\section{Reaction information}

\subsection{Gram scale reaction:}
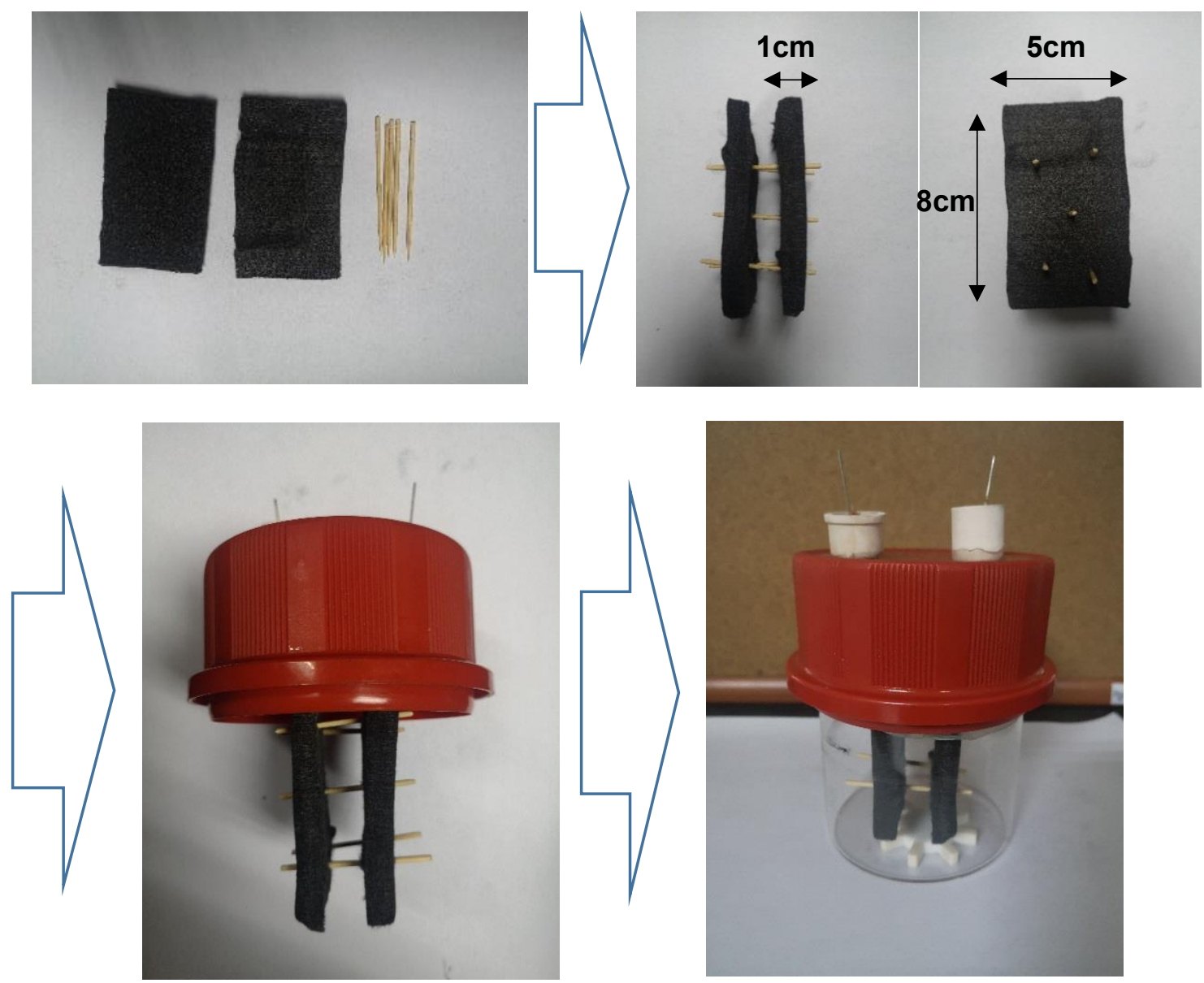

Figure S1. Reaction at 10-gram scale. 


\subsection{Numbers of reactants}<smiles>CC(C=O)c1ccccc1</smiles>

1 a<smiles>O=CCc1ccccc1</smiles>

$1 b$<smiles>O=CC1CCCC1</smiles>

$1 \mathrm{c}$<smiles>O=CC1CCCCC1</smiles>

1d<smiles>O=CC(c1ccccc1)c1ccccc1</smiles>

$1 e$<smiles>CC(C=O)Cc1ccc(C(C)C)cc1</smiles><smiles>CC(C=O)Cc1ccc(C(C)(C)C)cc1</smiles>

$1 \mathrm{~g}$<smiles>CC(C=O)Cc1ccc2c(c1)OCO2</smiles>

$1 \mathrm{k}$<smiles>CCCCCCCCCCCC=O</smiles>

$1 \mathrm{~h}$<smiles>Cc1ccc(CCC=O)o1</smiles>

11<smiles>CC(CC=O)CC(C)(C)C</smiles>

$1 \mathbf{i}$<smiles>CC(CC=O)CCCC(C)(C)O</smiles>

$1 \mathrm{~m}$

$1 \mathrm{j}$<smiles>O=CC1CC[Nb](=O)(O)C1</smiles>

$\bigcup_{\mathrm{CHO}}^{\mathrm{N}^{-\mathrm{BOC}}}$

$1 q$<smiles>O=CC1CCC[NH+]1[18O]</smiles>

$1 \mathrm{n}$

10

1p<smiles>Cc1cccc(C=O)c1</smiles><smiles>Cc1ccc(C=O)cc1</smiles>

$1 \mathrm{r}$<smiles>O=CC1CC=CCC1</smiles><smiles>Cc1ccccc1C=O</smiles>

$1 \mathrm{t}$

$1 v$<smiles>O=Cc1ccc2c(c1)CCO2</smiles>

$1 \mathrm{aa}$
$\mathrm{NC}$<smiles>N#Cc1ccc(C=O)cc1</smiles>

$1 w$<smiles>O=Cc1ccc(C(F)(F)F)cc1</smiles>

$1 x$<smiles>COc1ccc(C=O)cc1OC</smiles>

$1 y$<smiles>O=Cc1cc(F)ccc1F</smiles>

$1 z$<smiles>COc1ccc(CC(C)=O)cc1</smiles>

1 ae<smiles>O=Cc1csc2ccccc12</smiles>

$1 a b$<smiles>O=Cc1ccc2ccccc2n1</smiles>

1 ac<smiles>CCCCCCCCCC(C)=O</smiles>

$1 \mathrm{ad}$

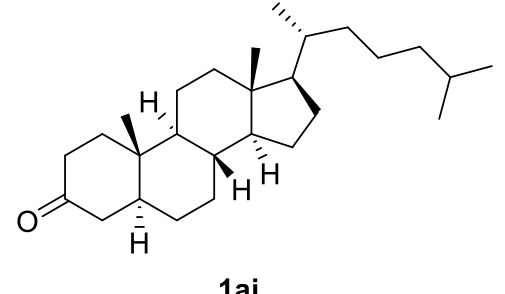

1 ag<smiles>CC(=O)c1ccccc1</smiles>

1 ah<smiles>O=C1CCCCC1</smiles>

1 af

Figure S2. Substrate applied in the reaction 


\subsection{Additional optimization reaction}

Table S1. Additional optimization results

\begin{tabular}{ccccc}
\hline Entry & Solvent & Electrolyte & base & yield \\
\hline 1 & DMF & $\mathrm{LiClO}_{4}(1.5 \mathrm{eq})$ & $\mathrm{LiOCH}_{3}$ & $78 \%(68 \%)$ \\
2 & $\mathrm{DMF}$ & $\mathrm{LiClO}_{4}(1.0 \mathrm{eq})$ & $\mathrm{LiOCH}_{3}$ & $69 \%$ \\
3 & $\mathrm{DMF}$ & $\mathrm{LiClO}_{4}(0.5 \mathrm{eq})$ & $\mathrm{LiOCH}_{3}$ & $23 \%$ \\
\hline
\end{tabular}

Condition: 1a $(0.2 \mathrm{mmol}), 2 \mathrm{a}(0.24 \mathrm{mmol})$, supporting electrolyte $(0.3 \mathrm{mmol})$, base $(0.04$ mmol), solvent ( $5 \mathrm{~mL})$, graphite felt electrodes as anode and cathode, gaseous $\mathrm{NH}_{3}$ in balloon, $5 \mathrm{~V}$ cell voltage, rt, $8 \mathrm{~h}$.

Table S2. Comparison of different hydrogen source

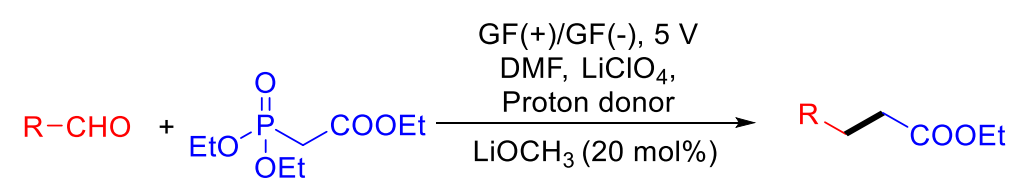

\begin{tabular}{|c|c|c|}
\hline $\mathrm{R}-\mathrm{CHO}$ & Proton donor & Yield \\
\hline \multirow{3}{*}{$\mathrm{CH}$} & ammonia & $78 \%$ \\
\hline & water & $56 \%$ \\
\hline & aqueous ammonia & was observed in GC-MS by-product such as \\
\hline \multirow[b]{2}{*}{$\mathrm{CHO}$} & ammonia & $50 \%$ \\
\hline & water & $43 \%$ \\
\hline \multirow{2}{*}{$\mathrm{CHC}$} & ammonia & $48 \%$ \\
\hline & water & $20 \%$ \\
\hline \multirow{2}{*}{$\widetilde{C H O}^{\mathrm{CHO}}$} & ammonia & $49 \%$ \\
\hline & water & $23 \%$ \\
\hline
\end{tabular}




\section{Investigation of reactants and intermediate}

\subsection{Detection of reaction intermediate 6}

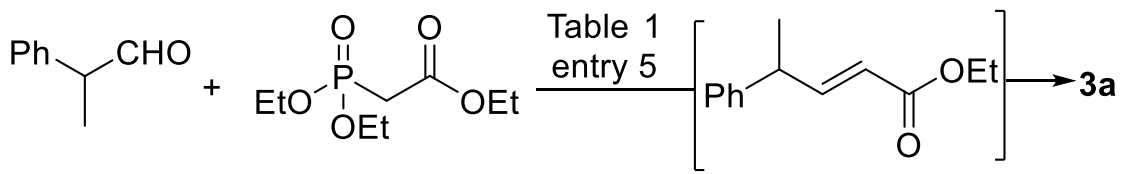

$1 \mathrm{a}$

$2 a$

6
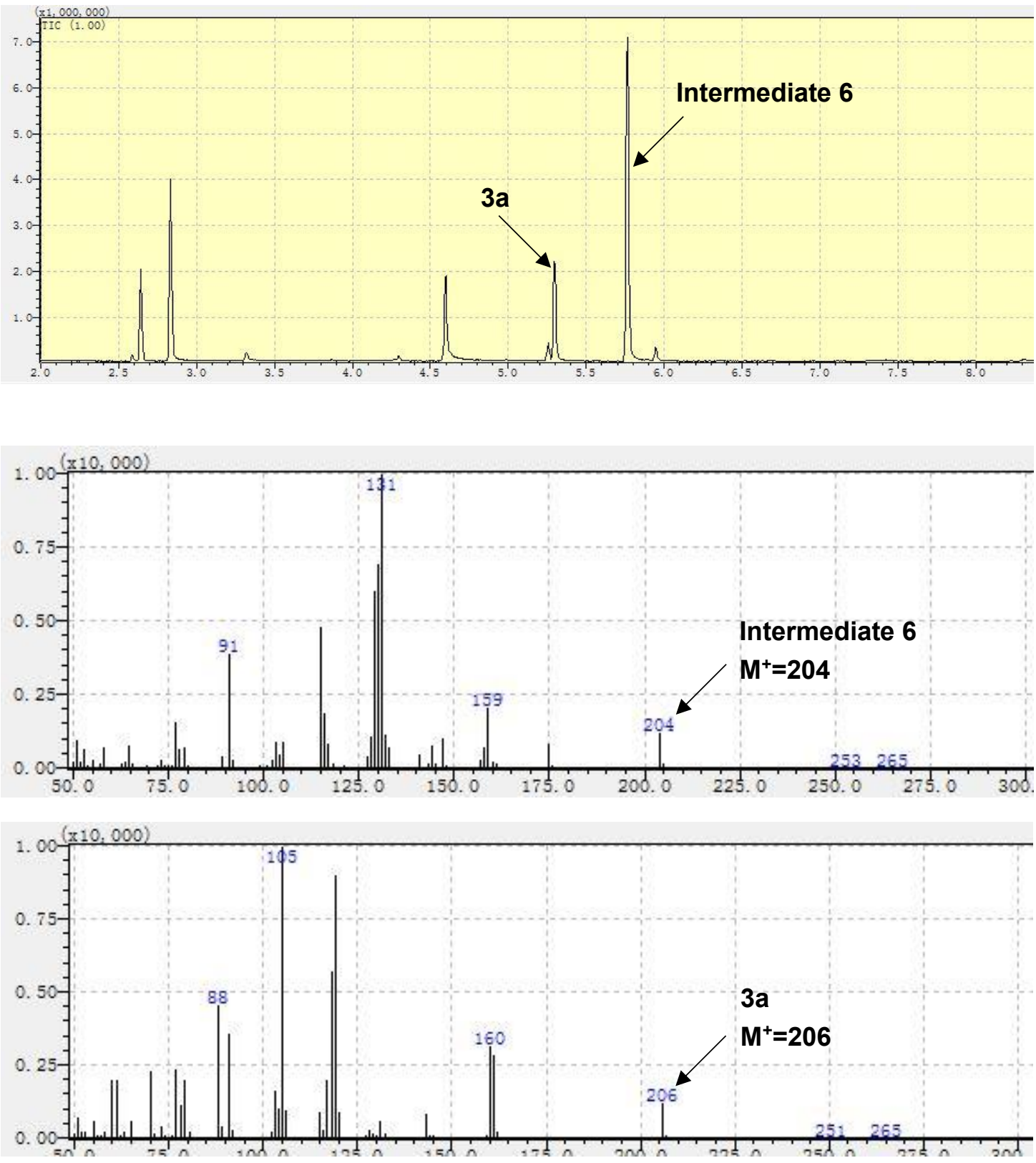

Figure S3. GC-MS chromatography and spectra of $\mathbf{1 a}$ and $\mathbf{2 a}$ 
2.2 Detection of side product in the reaction of $1 \mathrm{a}$ and $2 \mathrm{a}$ at the completion.

It was found the reduction of aldehyde to $1 \mathbf{a}$ was the significant side reaction. This was possible due to the slow condensation of 1a with 2a. In comparison, the condensation of 1a with more acidic $\mathbf{4}$ proceeded faster and the reduction of 1a was largely avoided.
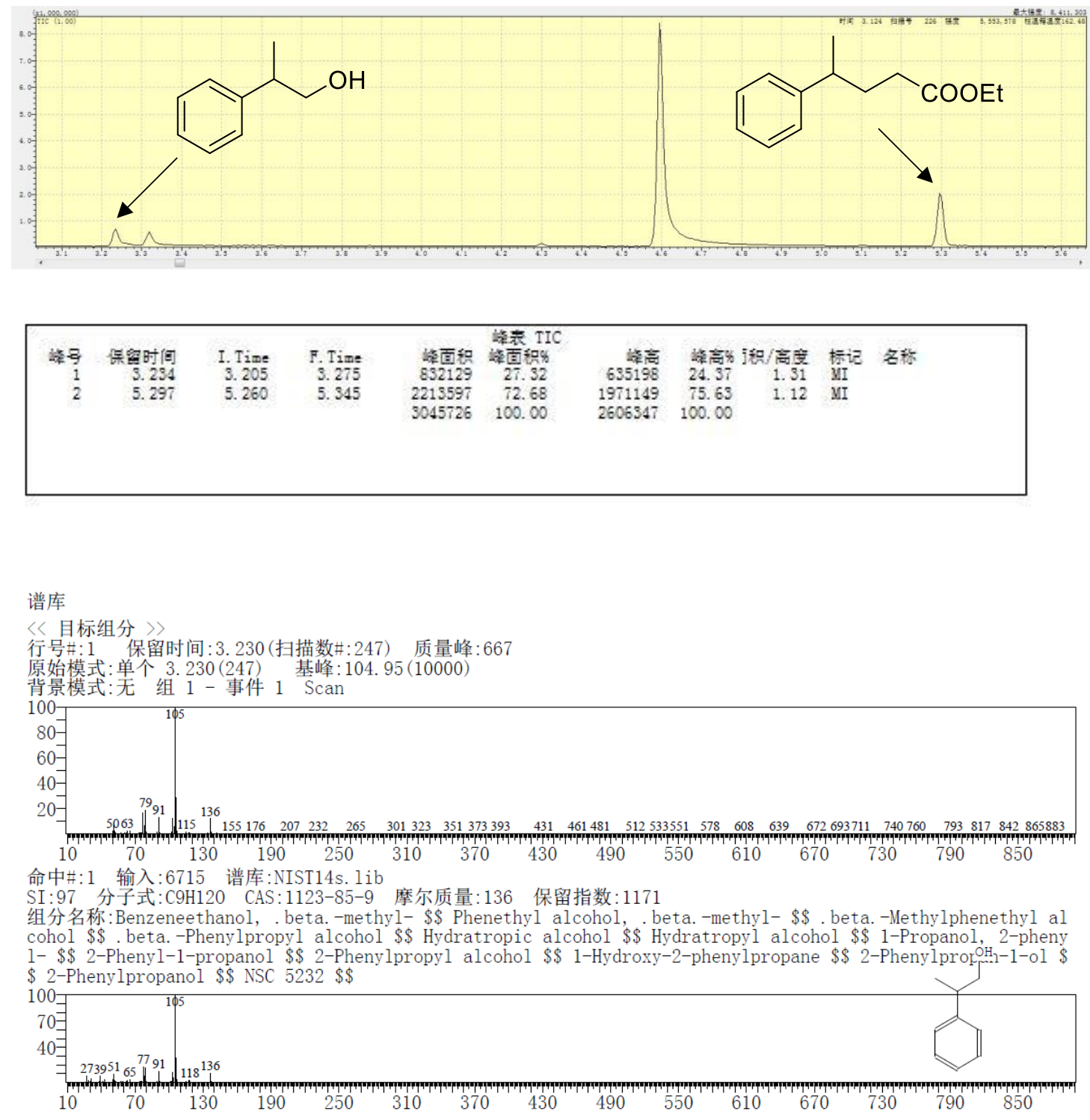

Figure S4. The detection of side product from reaction of $\mathbf{1 a}$ and $\mathbf{2 a}$ 


\subsection{Cyclic voltammetry experiment of anode}

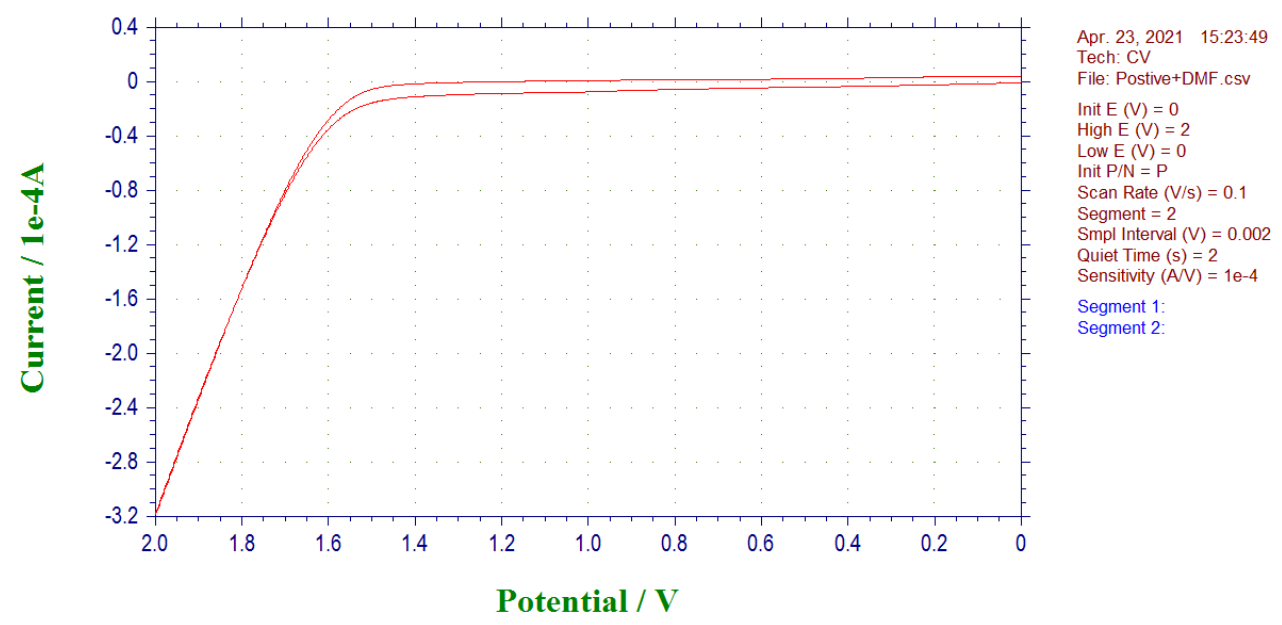

A) $\mathrm{DMF}$ (Blank)

A solution of $\mathrm{LiClO}_{4}(0.3 \mathrm{mmol})$ in $5 \mathrm{~mL}$ DMF was subject to the cyclic voltammetry experiment. Electrodes included a $4.0 \mathrm{~mm}$ glassy carbon working electrode, a gauze platinum counter electrode and a saturated calomel reference electrode (SCE) via a salt bridge charged with a solution of $\mathrm{LiClO}_{4}$ (0.02 M in DMF). Potential sweep rate was $100 \mathrm{mV} / \mathrm{s}$.

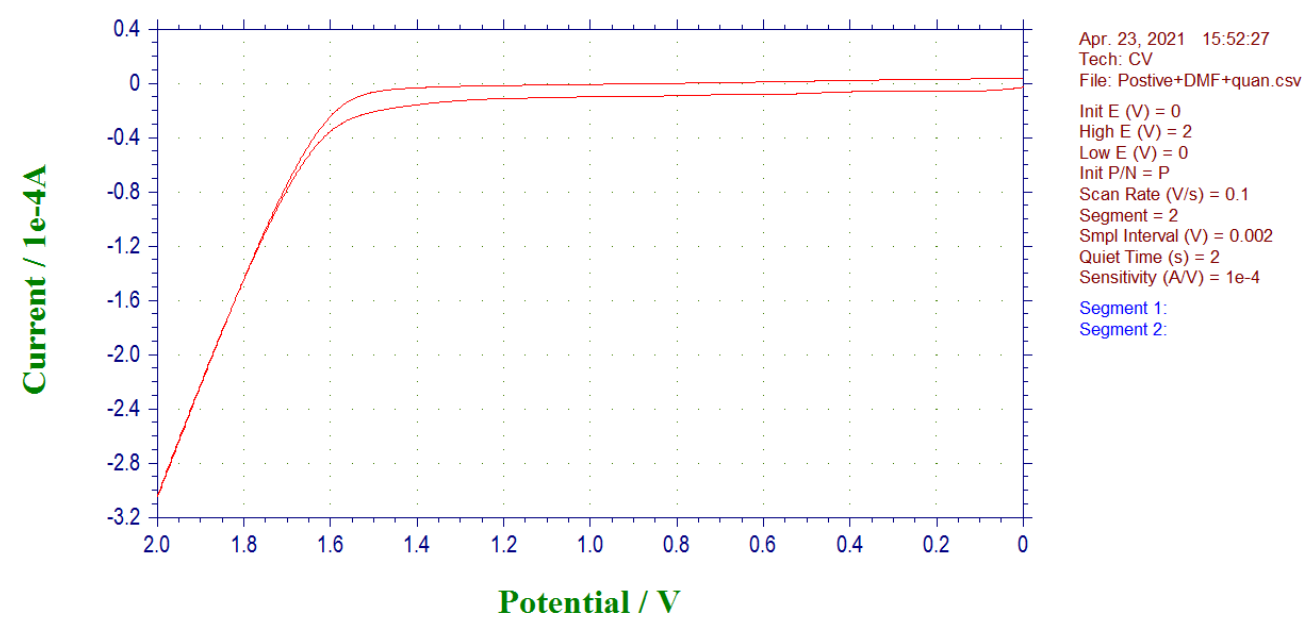

B) $1 \mathrm{a}$ in DMF

A solution of $\mathrm{LiClO}_{4}(0.3 \mathrm{mmol})$ and $1 \mathrm{a}(0.1 \mathrm{mmol})$ in $5 \mathrm{~mL}$ DMF was subject to the cyclic voltammetry experiment. Electrodes included a $4.0 \mathrm{~mm}$ glassy carbon working electrode, a gauze platinum counter electrode and a saturated calomel reference electrode (SCE) via a salt bridge charged with a solution of $\mathrm{LiClO}_{4}(0.02 \mathrm{M}$ in DMF). Potential sweep rate was $100 \mathrm{mV} / \mathrm{s}$. 


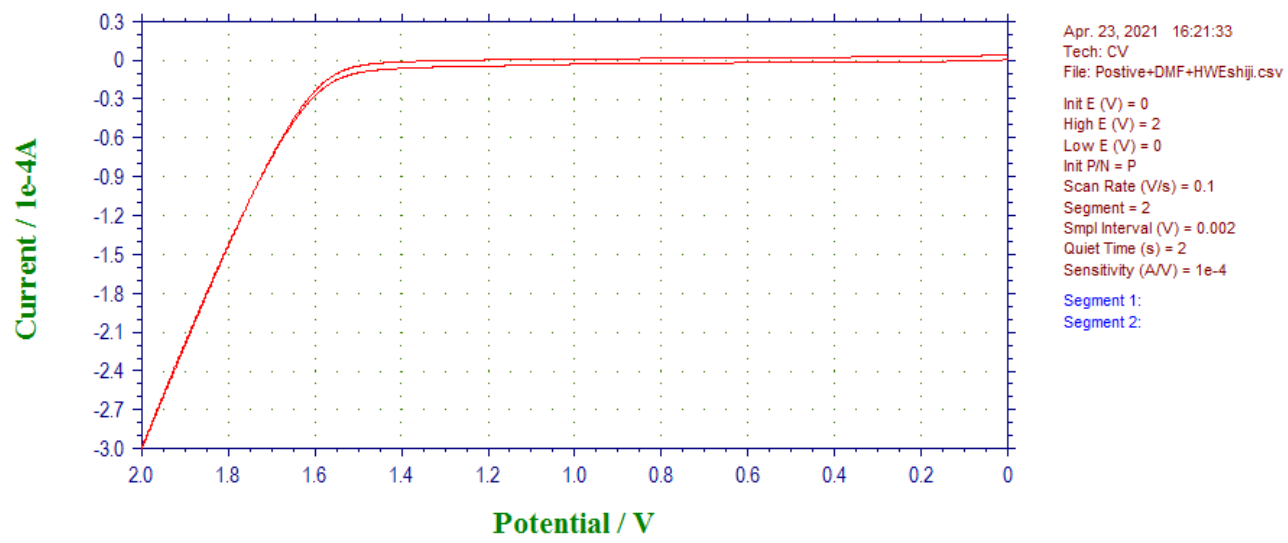

C) $2 \mathrm{a}$ in $\mathrm{DMF}$

A solution of $\mathrm{LiClO}_{4}(0.3 \mathrm{mmol})$ and $2 \mathrm{a}(0.1 \mathrm{mmol})$ in $5 \mathrm{~mL}$ DMF was subject to the cyclic voltammetry experiment. Electrodes included a $4.0 \mathrm{~mm}$ glassy carbon working electrode, a gauze platinum counter electrode and a saturated calomel reference electrode (SCE) via a salt bridge charged with a solution of $\mathrm{LiClO}_{4}(0.02 \mathrm{M}$ in DMF). Potential sweep rate was $100 \mathrm{mV} / \mathrm{s}$.

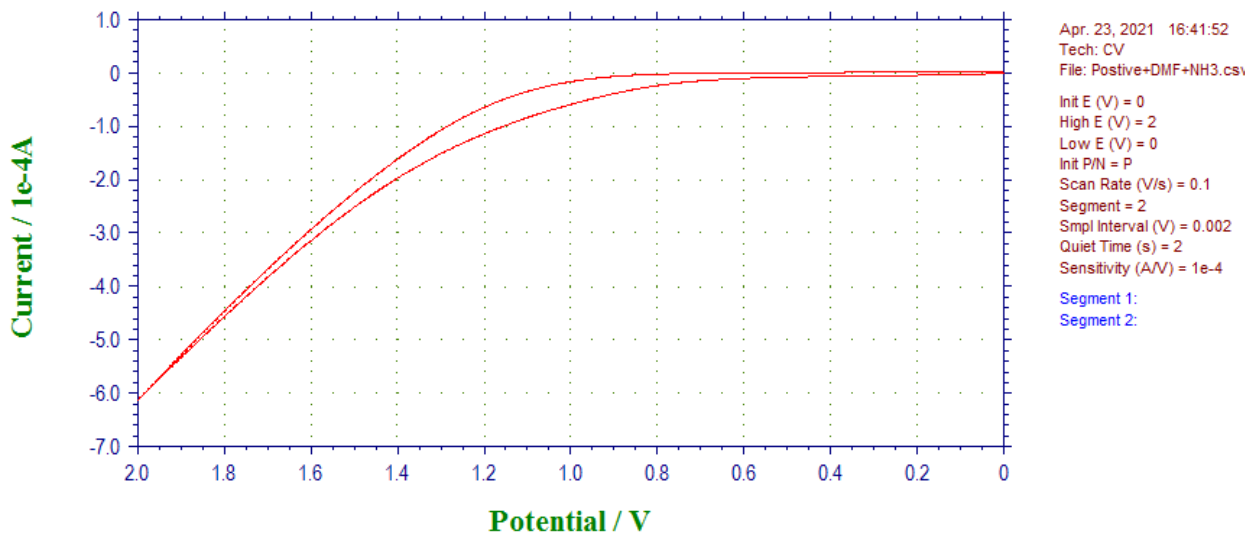

D) $\mathrm{NH}_{3}$ in $\mathrm{DMF}$

A solution of $\mathrm{LiClO}_{4}(0.3 \mathrm{mmol})$ in $5 \mathrm{~mL}$ DMF bubbled with $\mathrm{NH}_{3}$ was subject to the cyclic voltammetry experiment. Electrodes included a $4.0 \mathrm{~mm}$ glassy carbon working electrode, a gauze platinum counter electrode and a saturated calomel reference electrode (SCE) via a salt bridge charged with a solution of $\mathrm{LiClO}_{4}(0.02 \mathrm{M}$ in DMF). Potential sweep rate was $100 \mathrm{mV} / \mathrm{s}$.

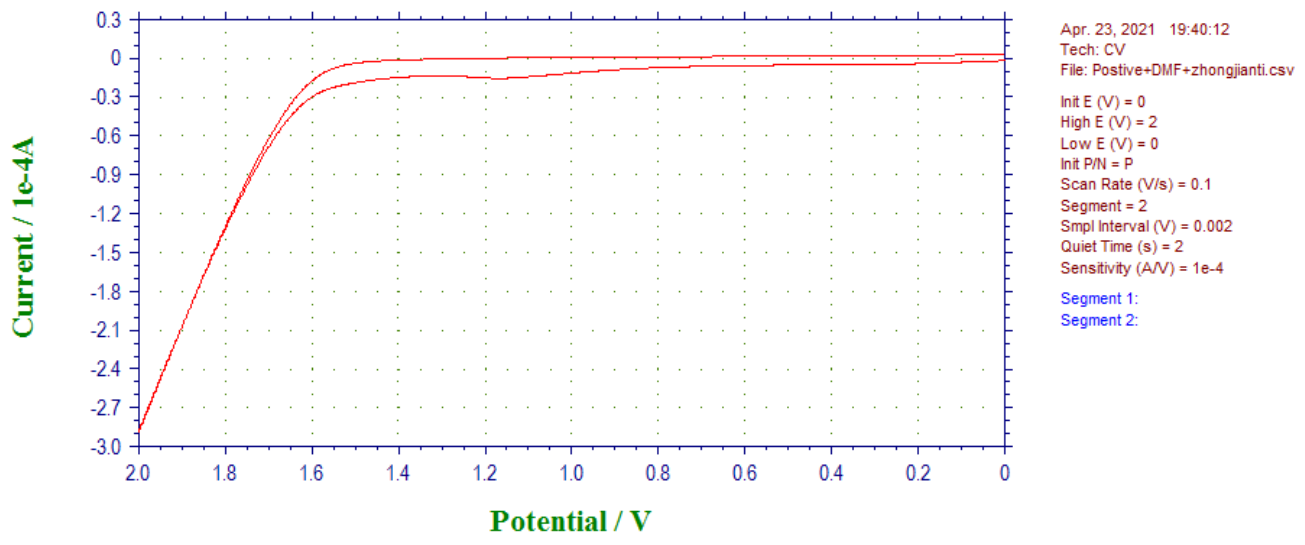

E) Intermediate 6 in DMF 
A solution of $\mathrm{LiClO}_{4}(0.3 \mathrm{mmol})$ and $6(0.1 \mathrm{mmol})$ in $5 \mathrm{~mL}$ DMF was subject to the cyclic voltammetry experiment. Electrodes included a $4.0 \mathrm{~mm}$ glassy carbon working electrode, a gauze platinum counter electrode and a saturated calomel reference electrode (SCE) via a salt bridge charged with a solution of $\mathrm{LiClO}_{4}(0.02 \mathrm{M}$ in DMF). Potential sweep rate was $100 \mathrm{mV} / \mathrm{s}$.

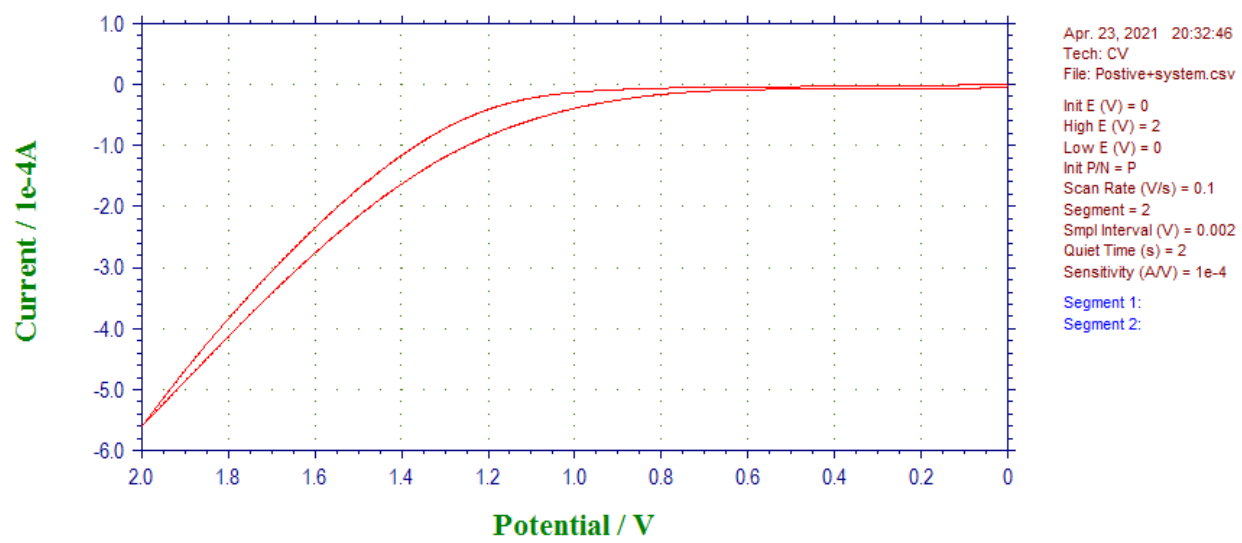

F) System

A solution of $\mathbf{1 a}(0.1 \mathrm{mmol}), \mathbf{2 a}(0.1 \mathrm{mmol}), \mathrm{MeOLi}(0.02 \mathrm{mmol})$ and $\mathrm{LiClO}_{4}(0.3 \mathrm{mmol})$ in $5 \mathrm{~mL}$ DMF bubbled with $\mathrm{NH}_{3}$ was subject to the cyclic voltammetry experiment. Electrodes included a $4.0 \mathrm{~mm}$ glassy carbon working electrode, a gauze platinum counter electrode and a saturated calomel reference electrode (SCE) via a salt bridge charged with a solution of $\mathrm{LiClO}_{4}(0.02 \mathrm{M}$ in DMF). Potential sweep rate was $100 \mathrm{mV} / \mathrm{s}$.

\subsection{Cyclic voltammetry experiment of cathode}

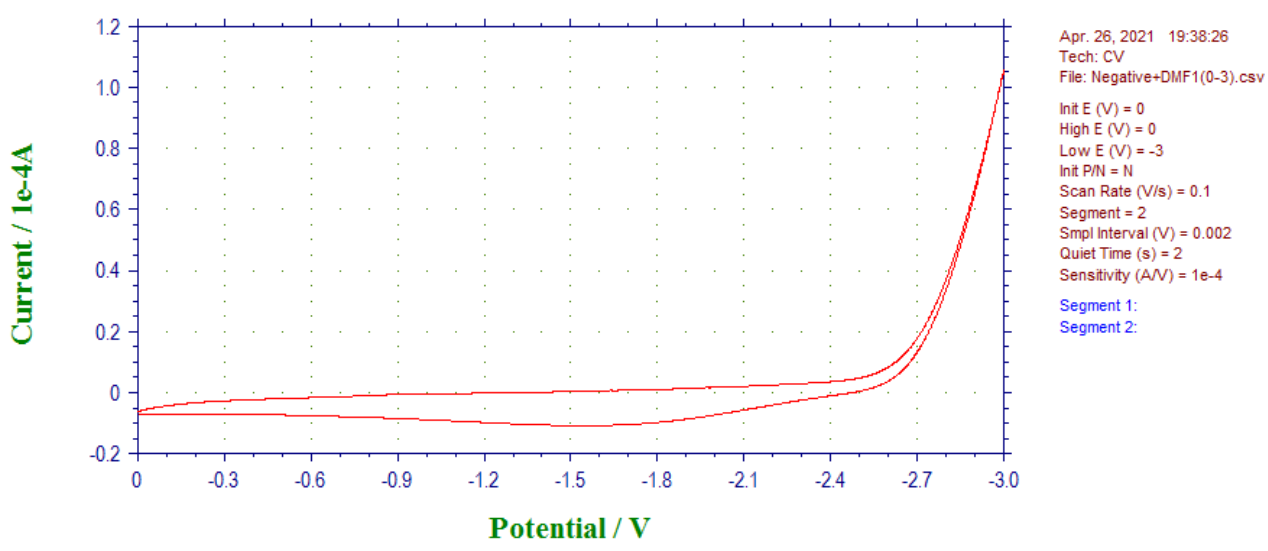

G) $\operatorname{DMF}($ Blank $)$

A solution of $\mathrm{LiClO}_{4}(0.3 \mathrm{mmol})$ in $5 \mathrm{~mL}$ DMF was subject to the cyclic voltammetry experiment. Electrodes included a $4.0 \mathrm{~mm}$ glassy carbon working electrode, a gauze platinum counter electrode and a saturated calomel reference electrode (SCE) via a salt bridge charged with a solution of $\mathrm{LiClO}_{4}$ (0.02 M in DMF). Potential sweep rate was $100 \mathrm{mV} / \mathrm{s}$. 


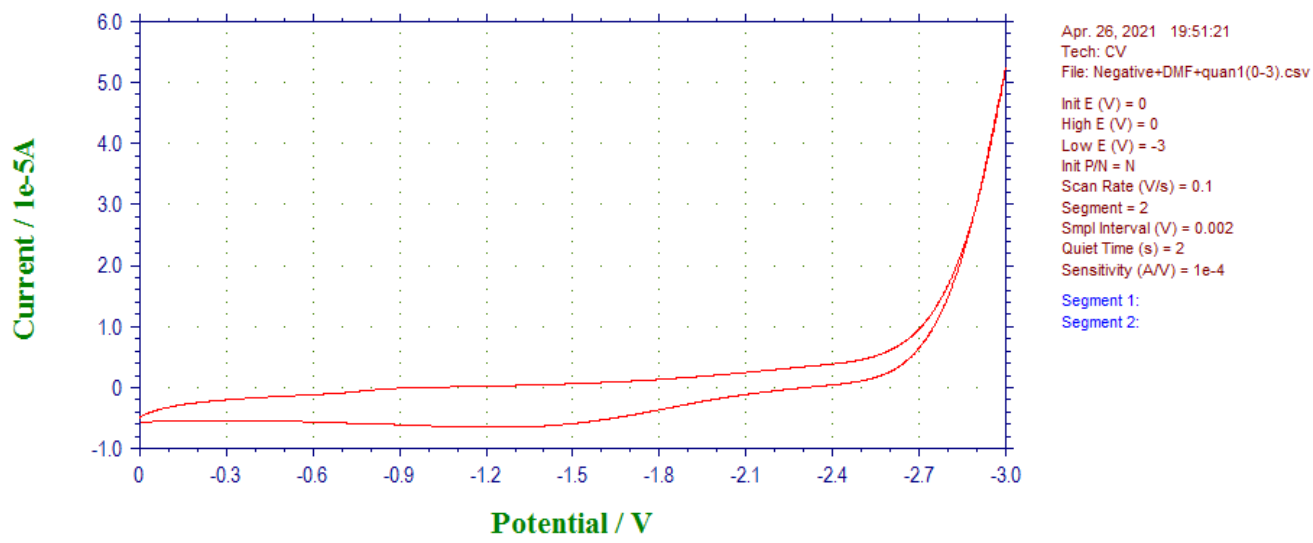

H) $1 \mathrm{a}$ in DMF

A solution of $\mathrm{LiClO}_{4}(0.3 \mathrm{mmol})$ and $1 \mathrm{a}(0.1 \mathrm{mmol})$ in $5 \mathrm{~mL}$ DMF was subject to the cyclic voltammetry experiment. Electrodes included a $4.0 \mathrm{~mm}$ glassy carbon working electrode, a gauze platinum counter electrode and a saturated calomel reference electrode (SCE) via a salt bridge charged with a solution of $\mathrm{LiClO}_{4}(0.02 \mathrm{M}$ in DMF). Potential sweep rate was $100 \mathrm{mV} / \mathrm{s}$.

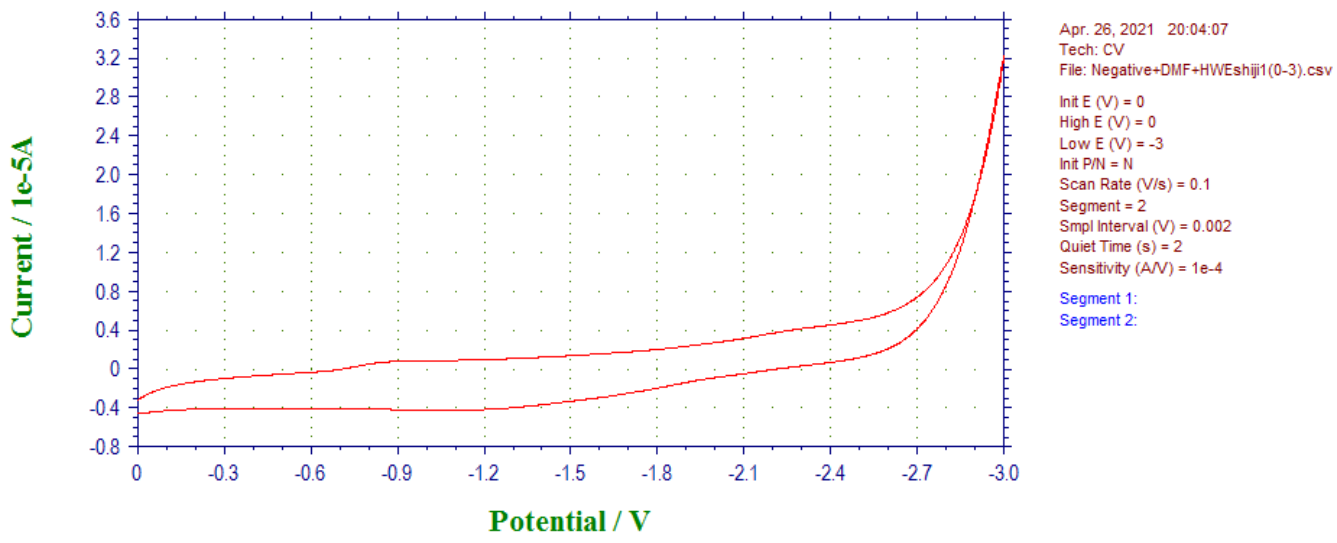

I) $2 \mathrm{a}$ in DMF

A solution of $\mathrm{LiClO}_{4}(0.3 \mathrm{mmol})$ and $2 \mathrm{a}(0.1 \mathrm{mmol})$ in $5 \mathrm{~mL}$ DMF was subject to the cyclic voltammetry experiment. Electrodes included a $4.0 \mathrm{~mm}$ glassy carbon working electrode, a gauze platinum counter electrode and a saturated calomel reference electrode (SCE) via a salt bridge charged with a solution of $\mathrm{LiClO}_{4}(0.02 \mathrm{M}$ in DMF). Potential sweep rate was $100 \mathrm{mV} / \mathrm{s}$.

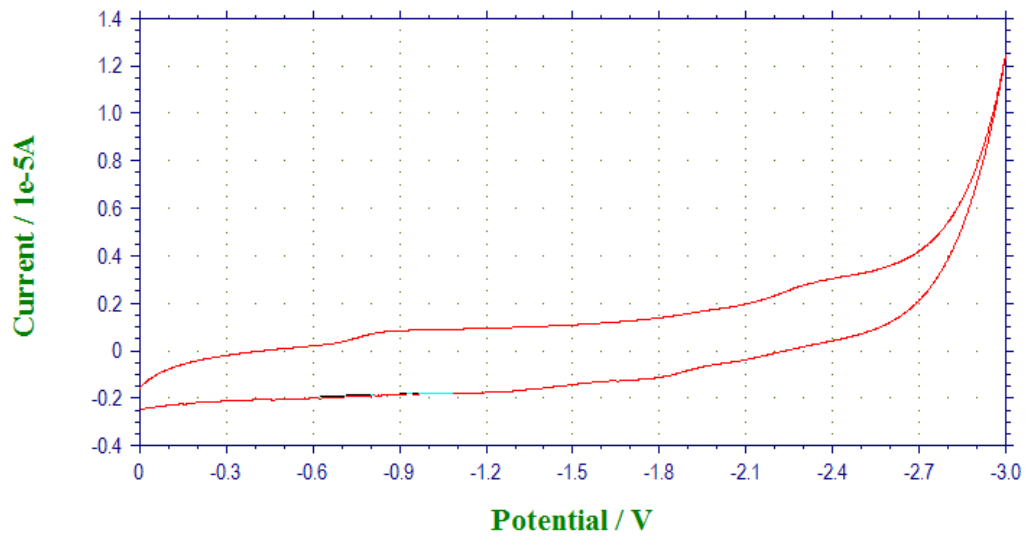

J) $\mathrm{NH}_{3}$ in $\mathrm{DMF}$
Apr. 26, 2021 21:37:51

File: Negative+DMF+NH3(dinongdu) 1(0-3)

$\ln i t E(V)=0$
$H \operatorname{High} E(V)=0$

Low $E(V)=-3$

Init P/N $=N$
Scan Rate $(V / s)=0.1$

Scan Rate (V/S)

Segment $=2$
Smpl Interval $(V)=0.002$

Quiet Time $(s)=2$

Sensitivity $(A N)=1 e-4$

Segment 1:

Segment 2:
$E p=-0.899 \mathrm{~V}$

$\mathrm{EP}=-6.0 .861 \mathrm{e}-8 \mathrm{~A}$

ip $=-6.561 e-8 A$
Ah $=-4.723 \mathrm{e}-8 \mathrm{C}$

$E p=-0.623 \mathrm{~V}$

ip $=-1.320 \mathrm{e}-7 \mathrm{~A}$
$\mathrm{Ah}=-1.133 \mathrm{e}-7 \mathrm{C}$ 
A solution of $\mathrm{LiClO}_{4}(0.3 \mathrm{mmol})$ in $5 \mathrm{~mL}$ DMF bubbled with $\mathrm{NH}_{3}$ was subject to the cyclic voltammetry experiment. Electrodes included a $4.0 \mathrm{~mm}$ glassy carbon working electrode, a gauze platinum counter electrode and a saturated calomel reference electrode (SCE) via a salt bridge charged with a solution of $\mathrm{LiClO}_{4}(0.02 \mathrm{M}$ in DMF). Potential sweep rate was $100 \mathrm{mV} / \mathrm{s}$.

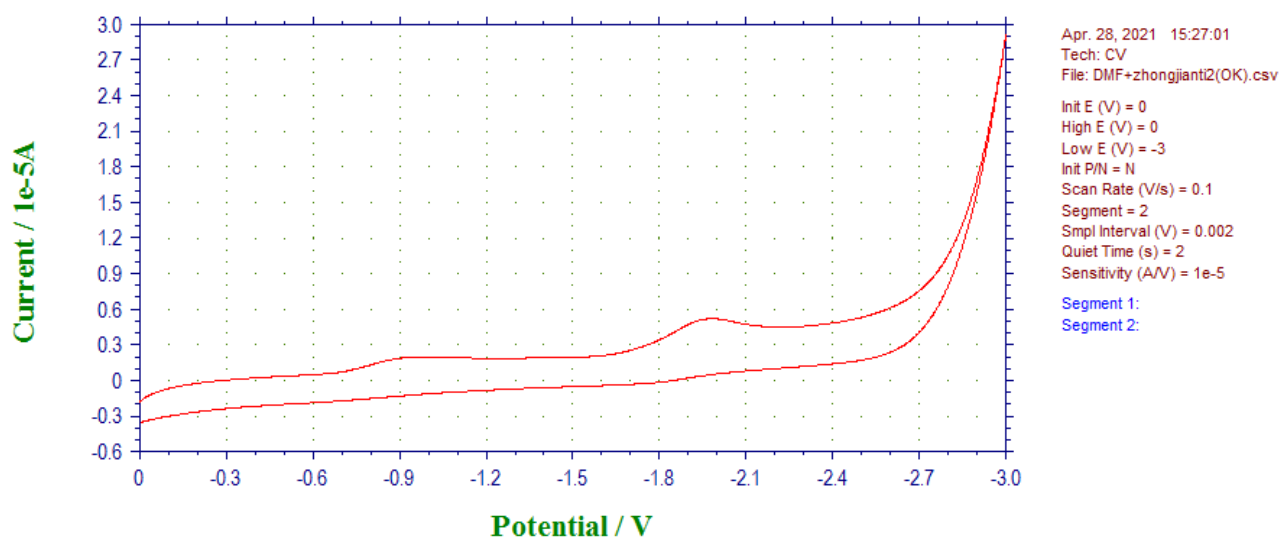

K) Intermediate 6 in DMF

A solution of $\mathrm{LiClO}_{4}(0.3 \mathrm{mmol})$ and $6(0.1 \mathrm{mmol})$ in $5 \mathrm{~mL}$ DMF was subject to the cyclic voltammetry experiment. Electrodes included a $4.0 \mathrm{~mm}$ glassy carbon working electrode, a gauze platinum counter electrode and a saturated calomel reference electrode (SCE) via a salt bridge charged with a solution of $\mathrm{LiClO}_{4}(0.02 \mathrm{M}$ in DMF). Potential sweep rate was $100 \mathrm{mV} / \mathrm{s}$.

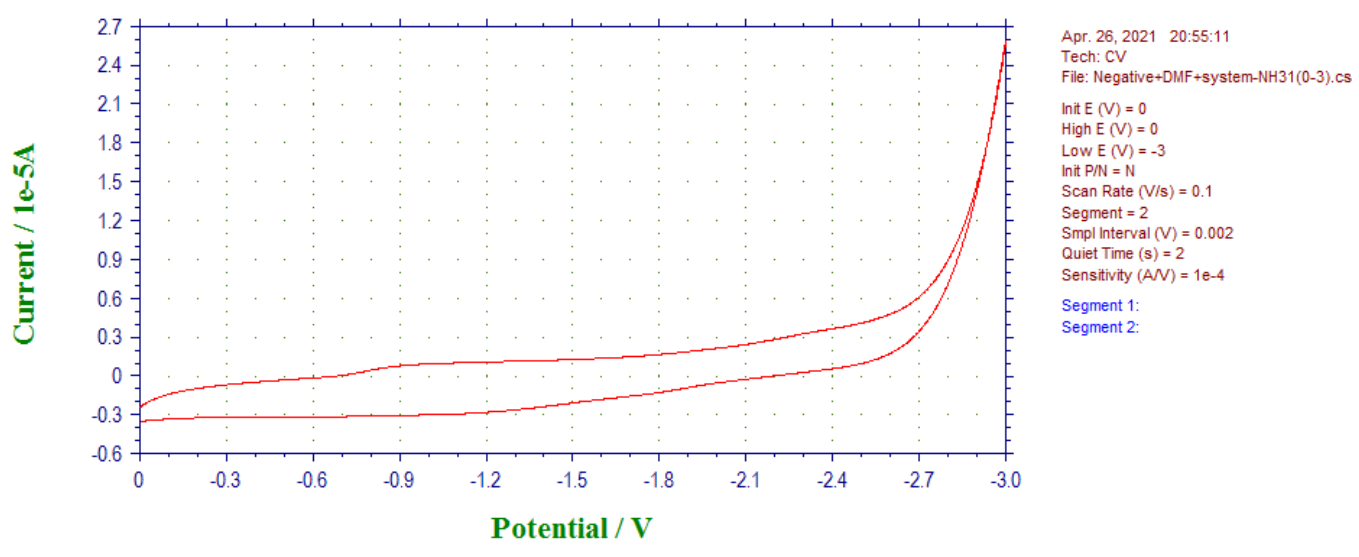

L) System

A solution of $\mathbf{1 a}(0.1 \mathrm{mmol}), \mathbf{2 a}(0.1 \mathrm{mmol}), \mathrm{MeOLi}(0.02 \mathrm{mmol})$ and $\mathrm{LiClO}_{4}(0.3 \mathrm{mmol})$ in $5 \mathrm{~mL}$ DMF bubbled with $\mathrm{NH}_{3}$ was subject to the cyclic voltammetry experiment. Electrodes included a $4.0 \mathrm{~mm}$ glassy carbon working electrode, a gauze platinum counter electrode and a saturated calomel reference electrode (SCE) via a salt bridge charged with a solution of $\mathrm{LiClO}_{4}(0.02 \mathrm{M}$ in DMF). Potential sweep rate was $100 \mathrm{mV} / \mathrm{s}$. 


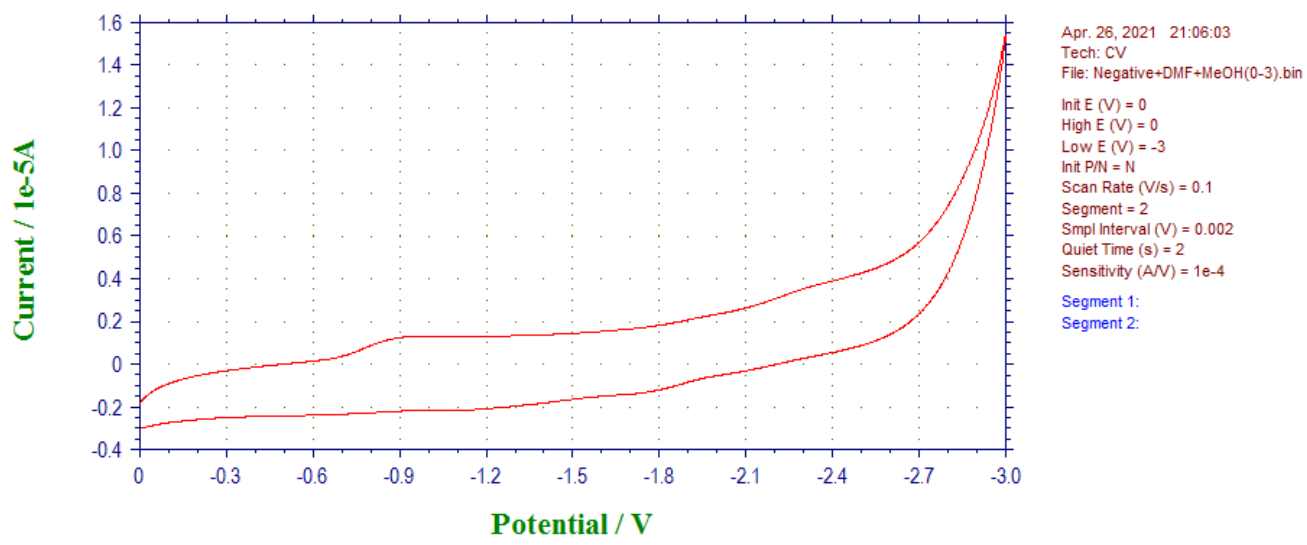

M) $\mathrm{MeOH}$ in $\mathrm{DMF}$

A solution of $\mathrm{MeOH}(0.2 \mathrm{mmol})$ in $5 \mathrm{~mL}$ DMF was subject to the cyclic voltammetry experiment. Electrodes included a $4.0 \mathrm{~mm}$ glassy carbon working electrode, a gauze platinum counter electrode and a saturated calomel reference electrode (SCE) via a salt bridge charged with a solution of $\mathrm{LiClO}_{4}$ (0.02 M in DMF). Potential sweep rate was $100 \mathrm{mV} / \mathrm{s}$. 


\section{NMR Spectra}

${ }^{1} \mathrm{H}$ NMR $\left(400 \mathrm{MHz}, \mathrm{CDCl}_{3}\right)$ of $\mathbf{3 a}$

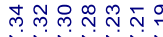

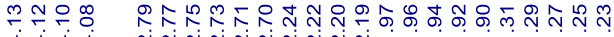

isivitis

A did
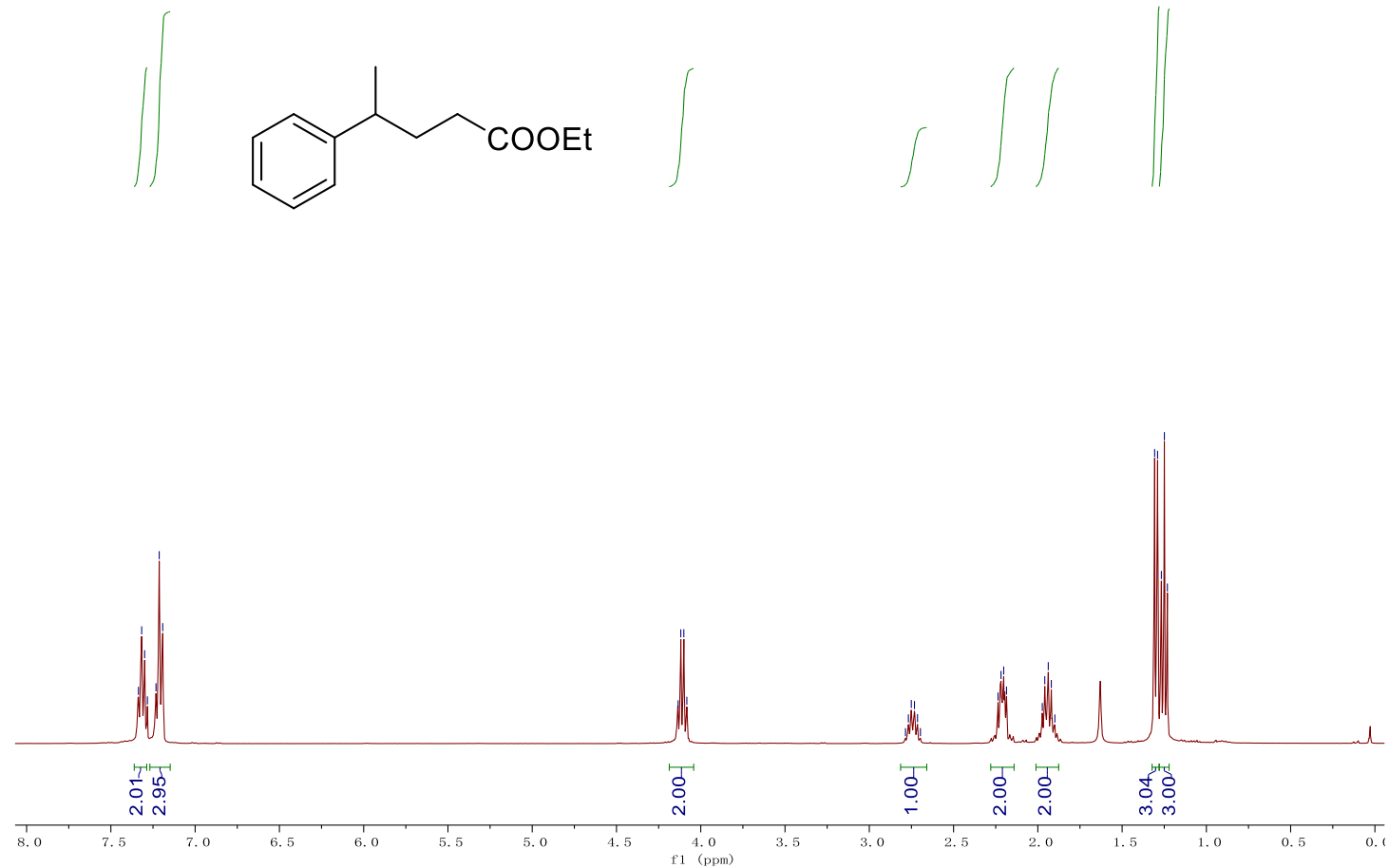

${ }^{13} \mathrm{C}\{1 \mathrm{H}\} \mathrm{NMR}\left(100 \mathrm{MHz}, \mathrm{CDCl}_{3}\right)$ of $\mathbf{3 a}$

\begin{tabular}{|c|c|c|c|c|}
\hline 6 & $\begin{array}{l}\bar{m} \\
\stackrel{0}{0} \\
\bar{j}\end{array}$ & 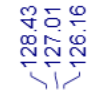 & $\stackrel{0}{\check{0}}$ & 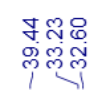 \\
\hline
\end{tabular}<smiles>CCOC(=O)CCC(C)c1ccccc1</smiles>

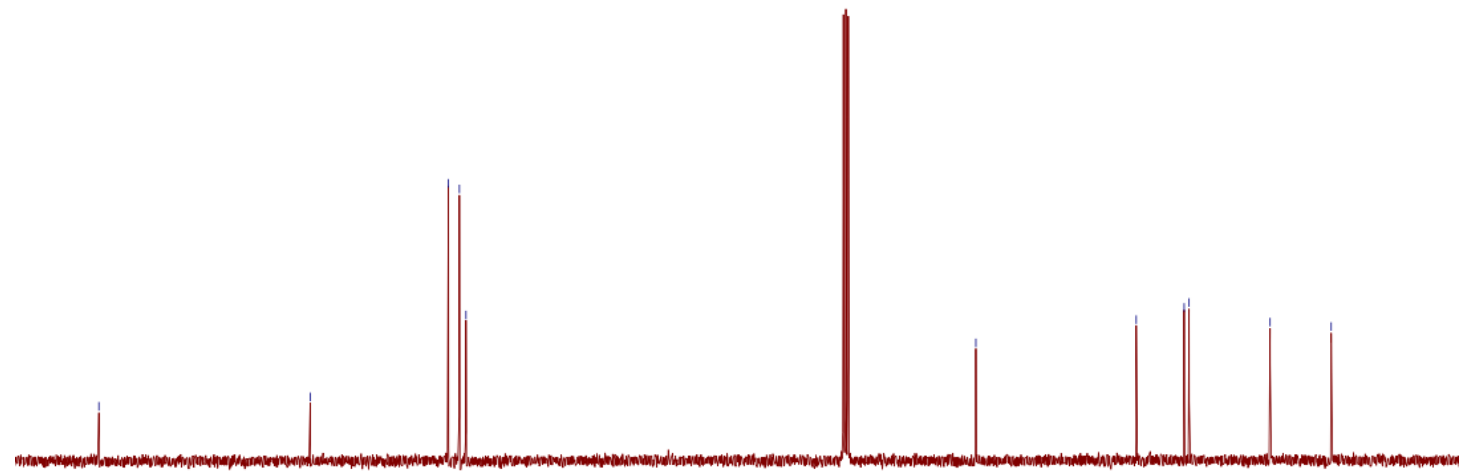

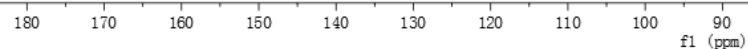


${ }^{1} \mathrm{H}$ NMR (400 MHz, $\left.\mathrm{CDCl}_{3}\right)$ of $\mathbf{3 b}$
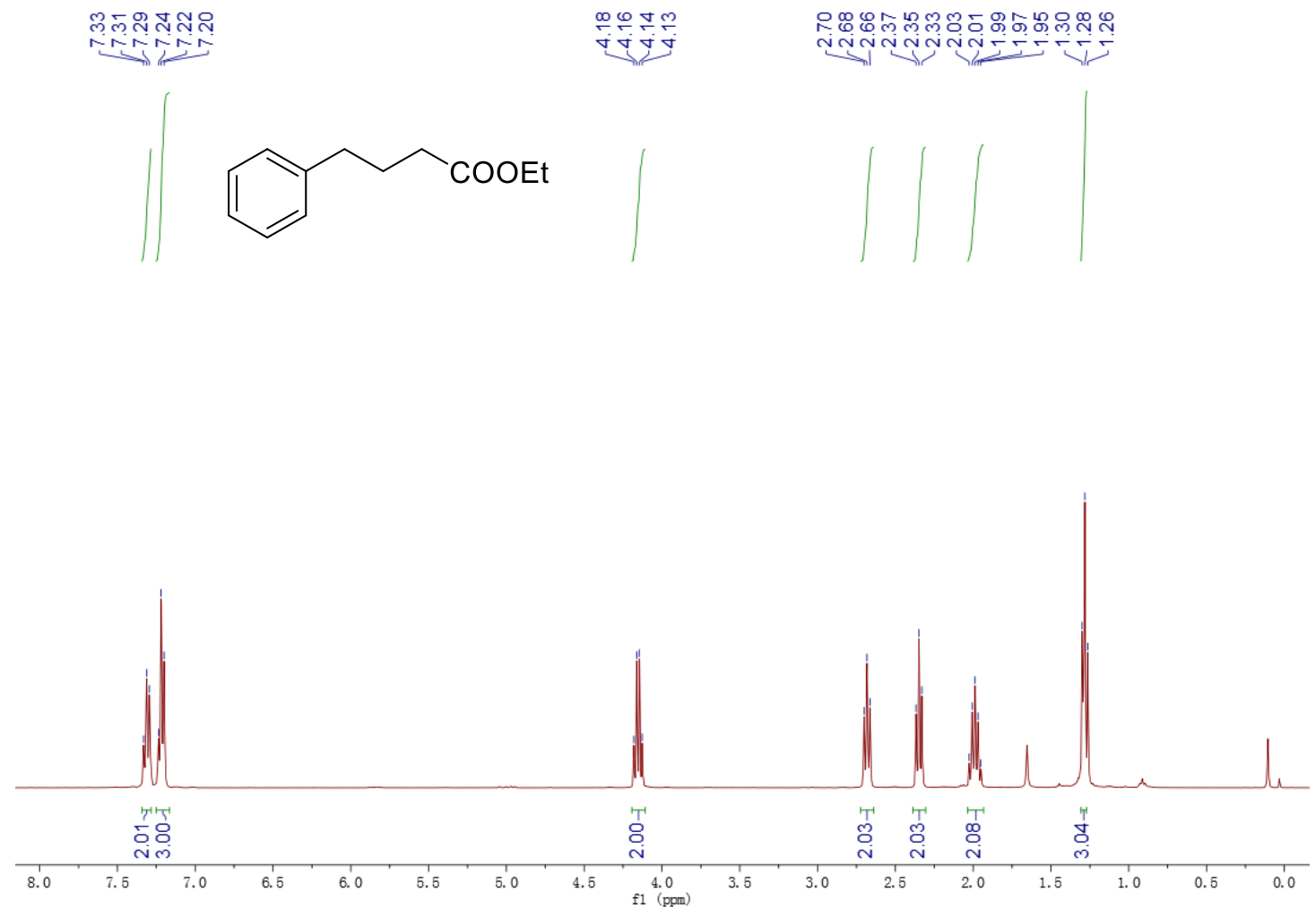

${ }^{13} \mathrm{C}\{1 \mathrm{H}\}$ NMR $\left(100 \mathrm{MHz}, \mathrm{CDCl}_{3}\right)$ of $\mathbf{3 b}$

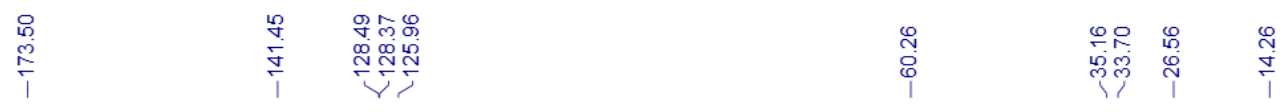<smiles>CCOC(=O)CCCc1ccccc1</smiles>
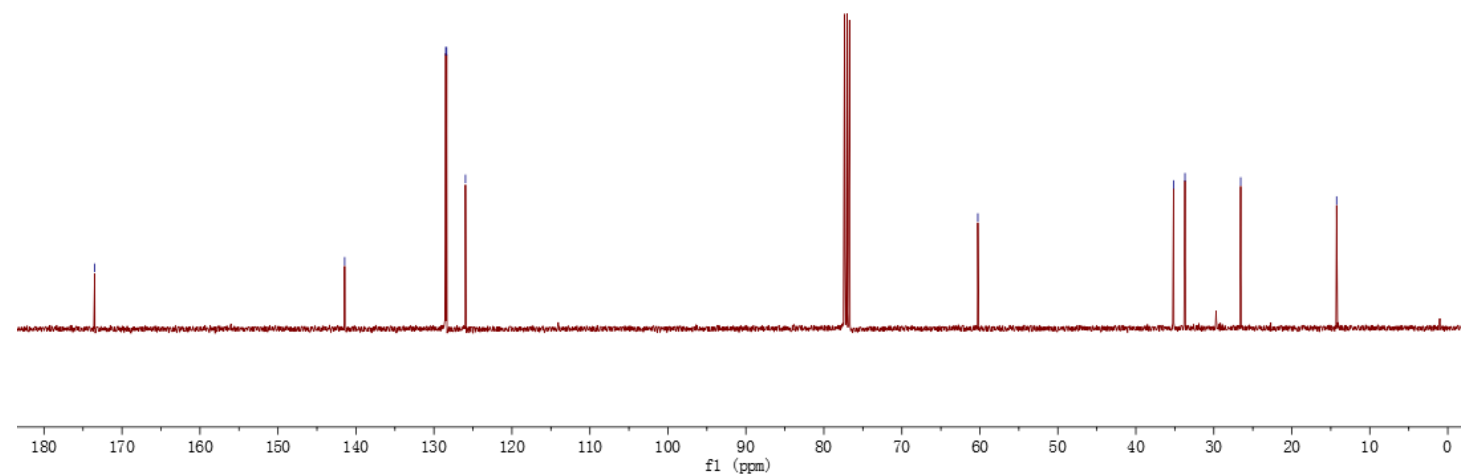
${ }^{1} \mathrm{H}$ NMR $\left(400 \mathrm{MHz}, \mathrm{CDCl}_{3}\right)$ of $\mathbf{3 c}$
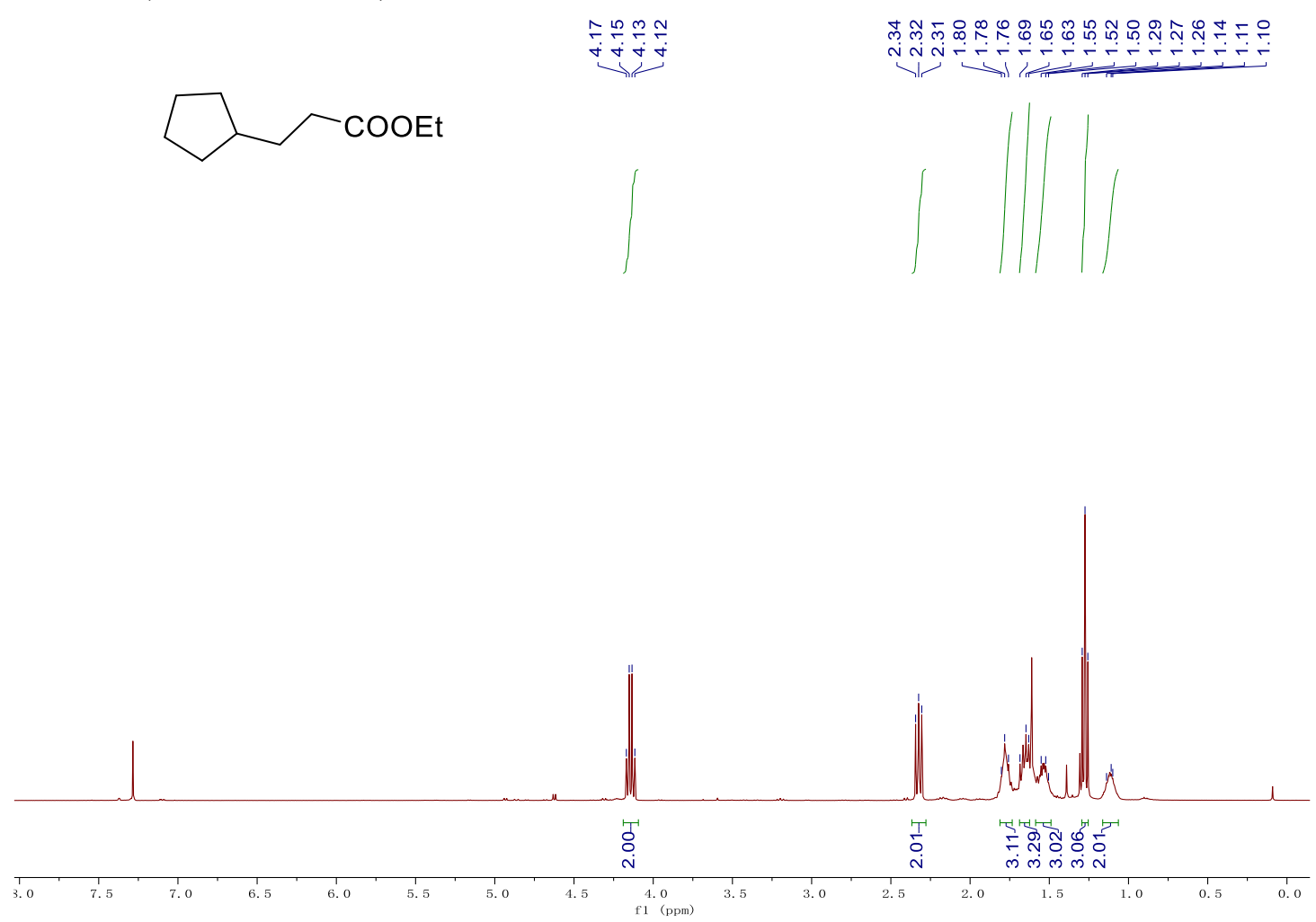

${ }^{13} \mathrm{C}\{1 \mathrm{H}\}$ NMR $\left(100 \mathrm{MHz}, \mathrm{CDCl}_{3}\right)$ of $\mathbf{3 c}$

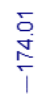

ग.<smiles>CCOC(=O)CCC1CCCC1</smiles>

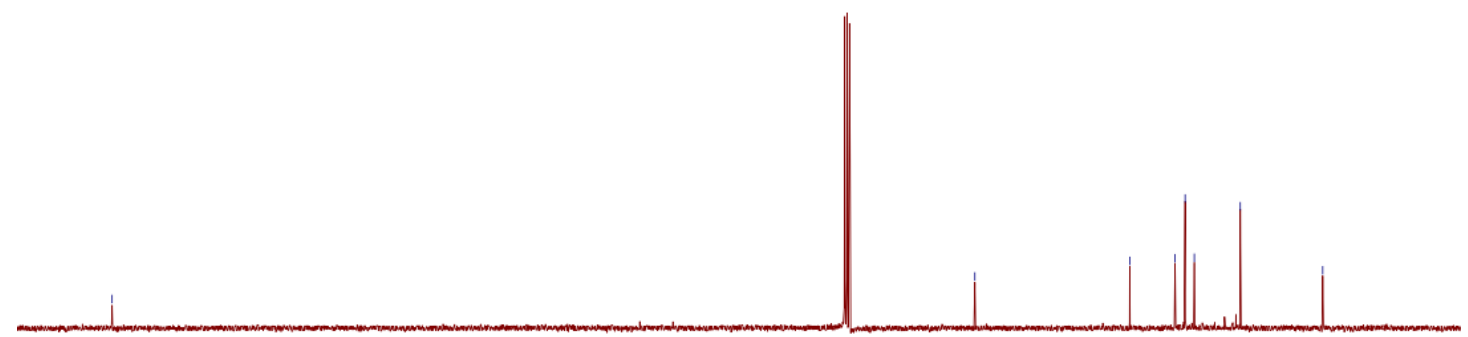

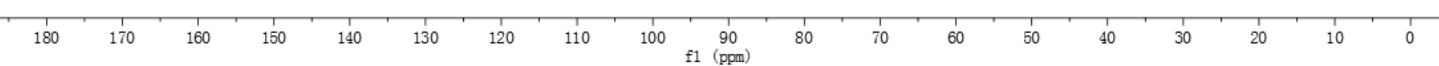


${ }^{1} \mathrm{H}$ NMR (400 MHz, $\mathrm{CDCl}_{3}$ ) of $\mathbf{3 d}$

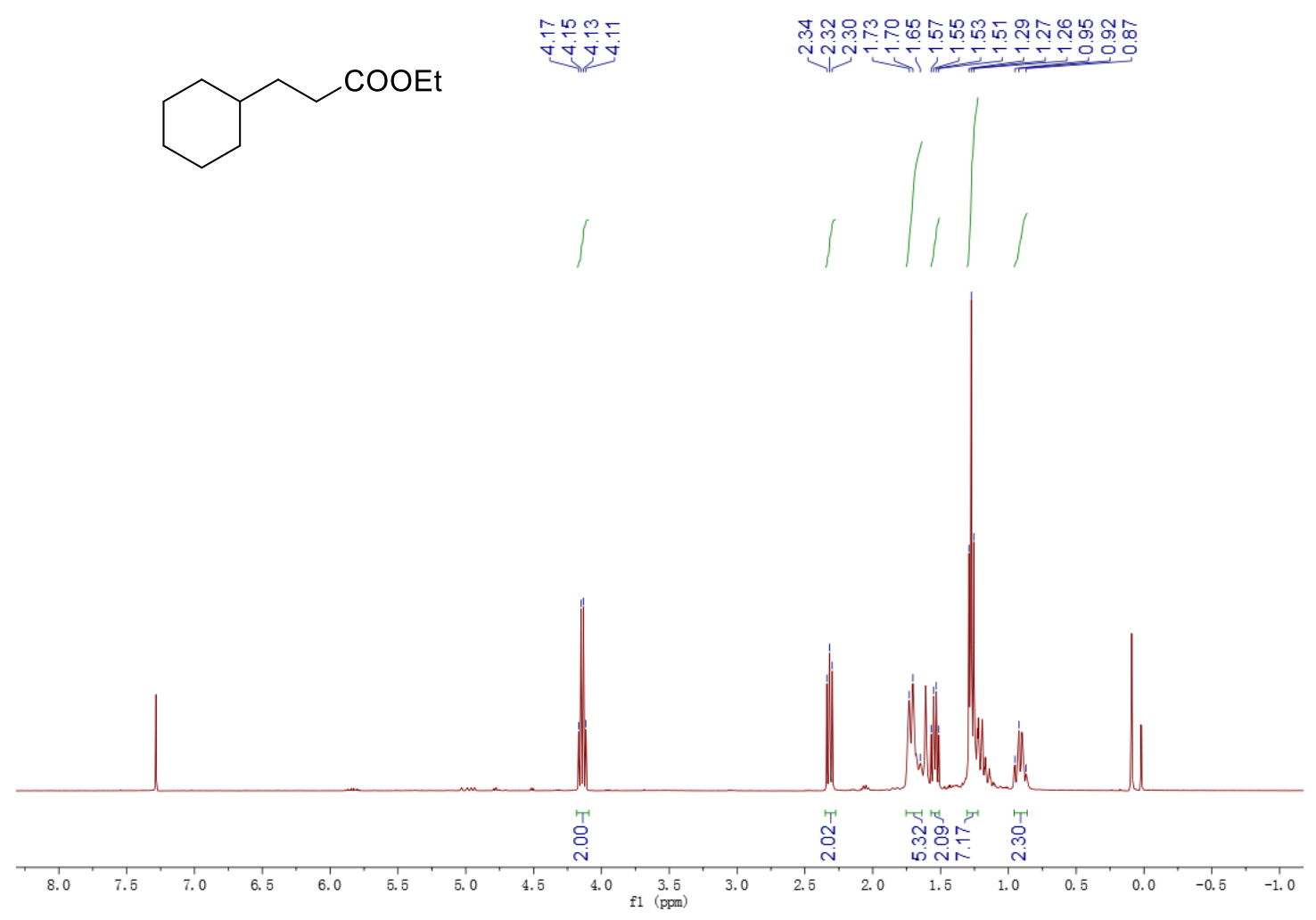

${ }^{13} \mathrm{C}\{1 \mathrm{H}\} \mathrm{NMR}\left(100 \mathrm{MHz}, \mathrm{CDCl}_{3}\right)$ of $\mathbf{3 d}$<smiles>CCOC(=O)CCC1CCCCC1</smiles> 
${ }^{1} \mathrm{H}$ NMR (400 MHz, $\mathrm{CDCl}_{3}$ ) of $\mathbf{3 e}$

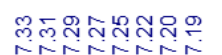

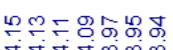

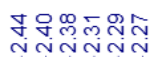

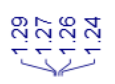

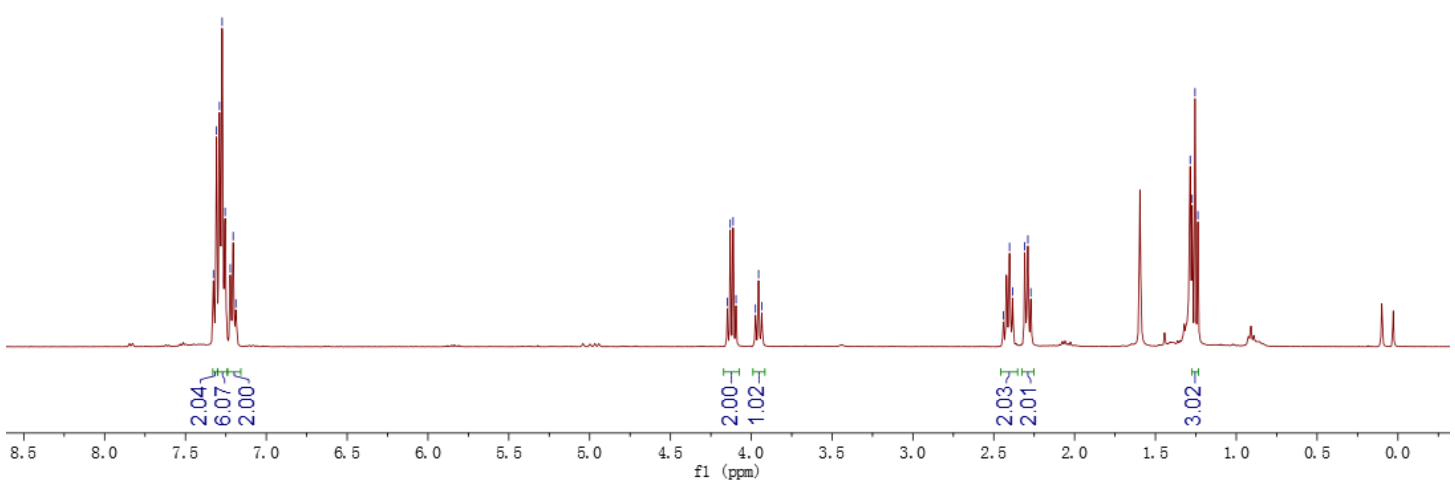

${ }^{13} \mathrm{C}\{1 \mathrm{H}\}$ NMR $\left(100 \mathrm{MHz}, \mathrm{CDCl}_{3}\right)$ of $\mathbf{3 e}$

芯

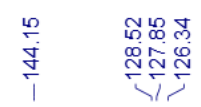

可

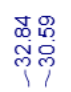

文
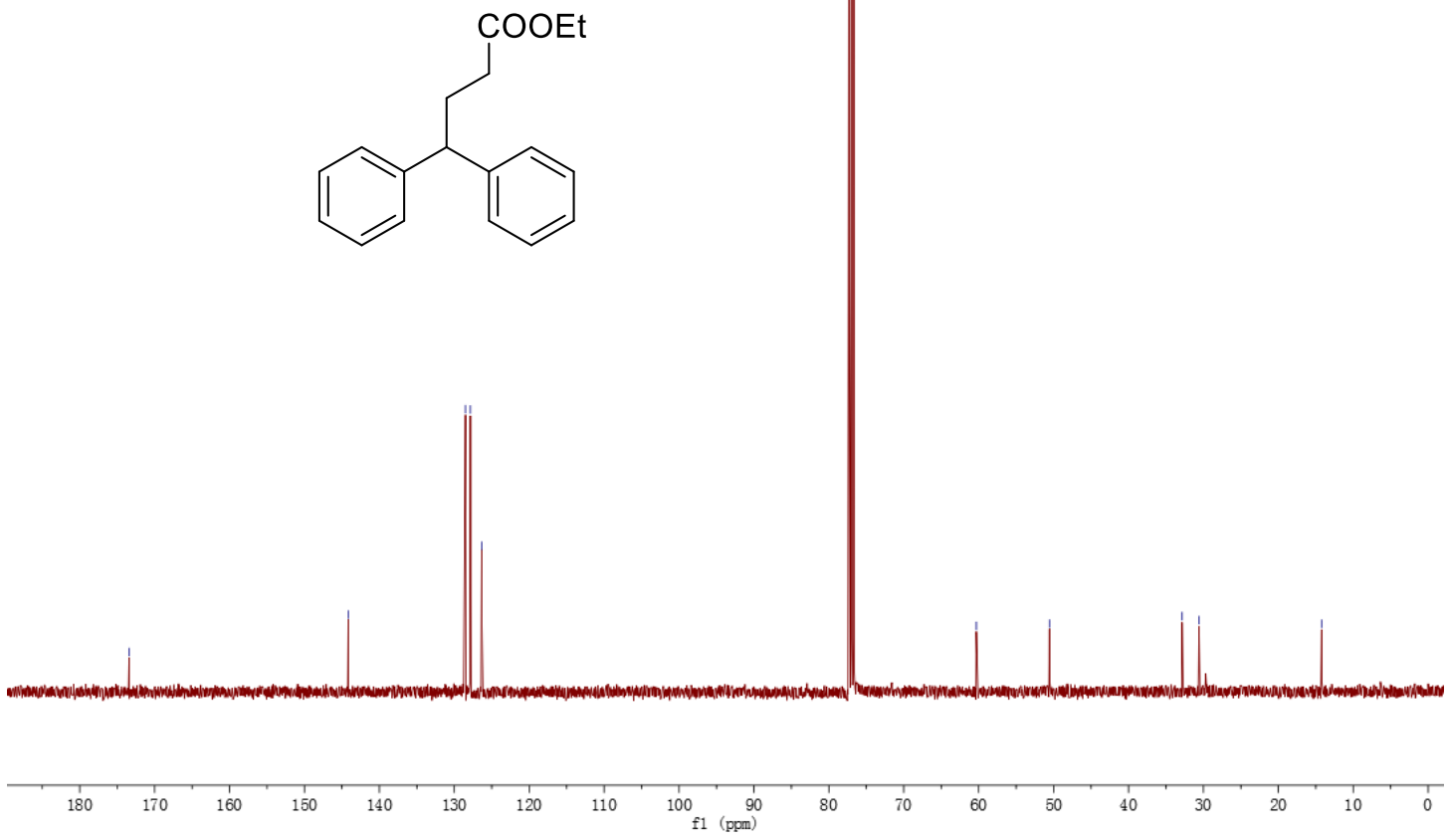

S18 
${ }^{1} \mathrm{H}$ NMR (400 MHz, $\left.\mathrm{CDCl}_{3}\right)$ of $\mathbf{3 f}$

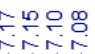

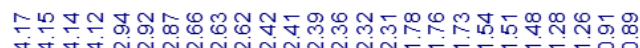

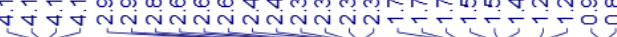

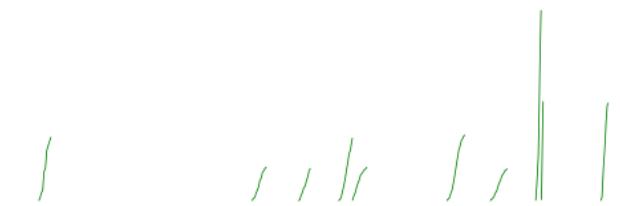<smiles>CCOC(=O)CCC(C)Cc1ccc(C(C)C)cc1</smiles>

hith the the

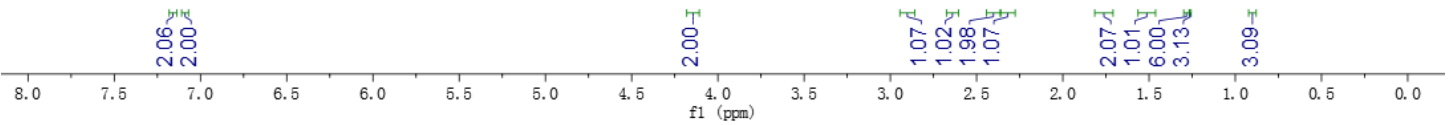

${ }^{13} \mathrm{C}\{1 \mathrm{H}\}$ NMR $\left(100 \mathrm{MHz}, \mathrm{CDCl}_{3}\right)$ of $\mathbf{3 f}$

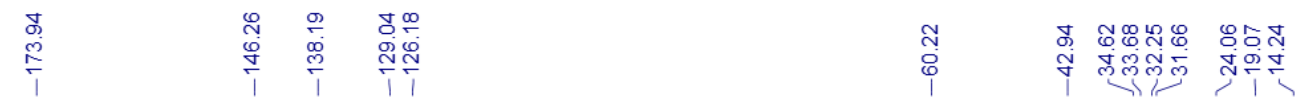<smiles>CCOC(=O)CCC(C)Cc1ccc(C(C)C)cc1</smiles>

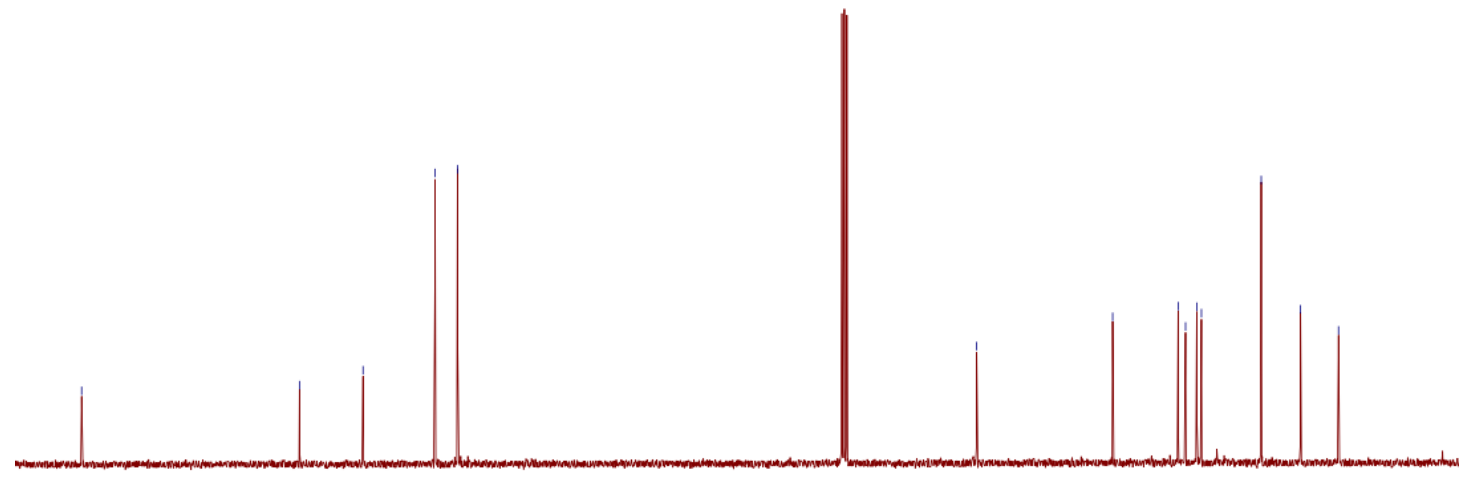

f1 $\stackrel{90}{(\mathrm{ppm})}$ 
${ }^{1} \mathrm{H}$ NMR (400 MHz, $\left.\mathrm{CDCl}_{3}\right)$ of $\mathbf{3 g}$

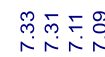

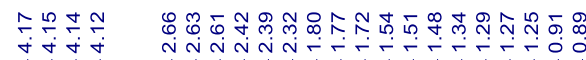

iñ

cootst

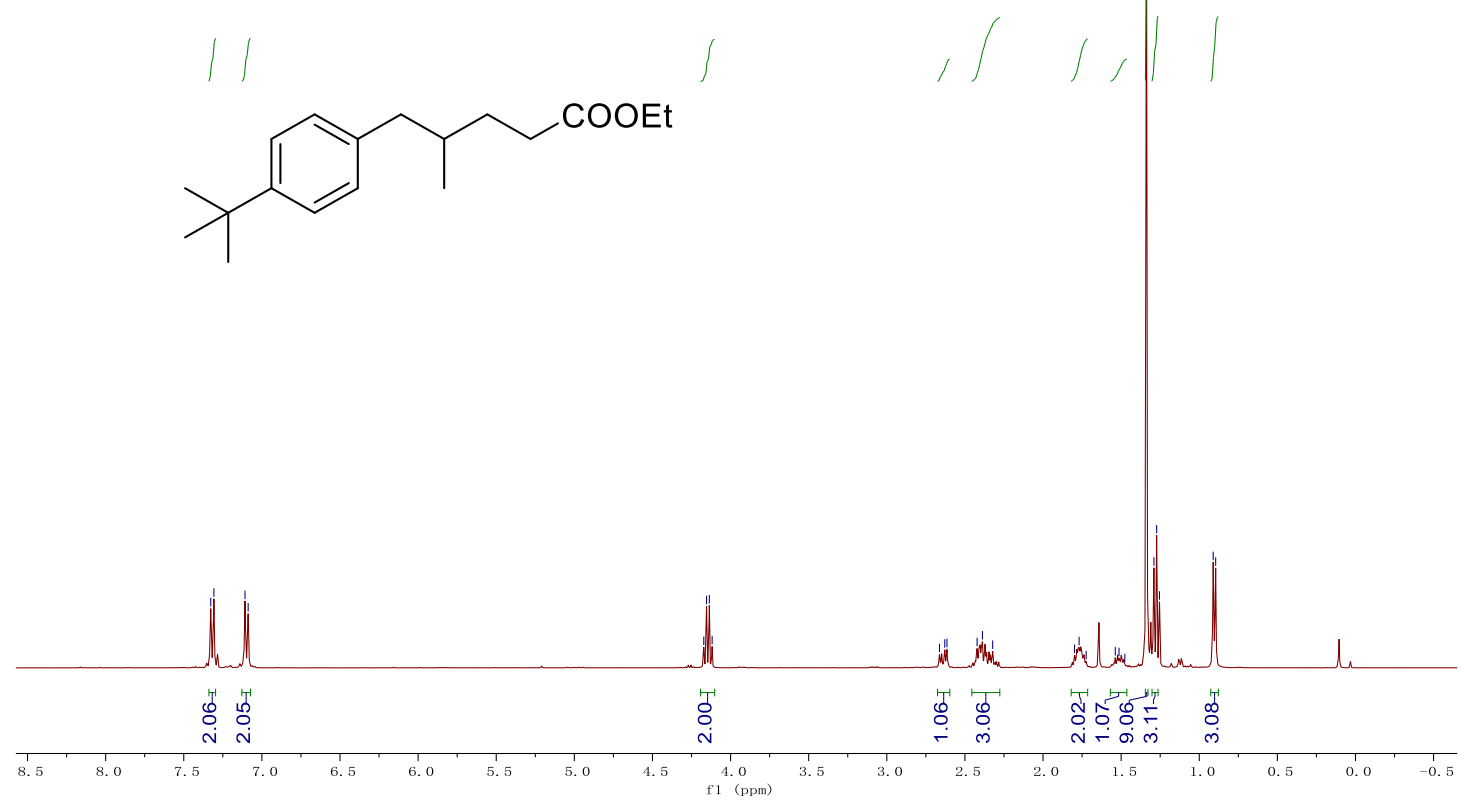

${ }^{13} \mathrm{C}\{1 \mathrm{H}\} \mathrm{NMR}\left(100 \mathrm{MHz}, \mathrm{CDCl}_{3}\right)$ of $\mathbf{3 g}$

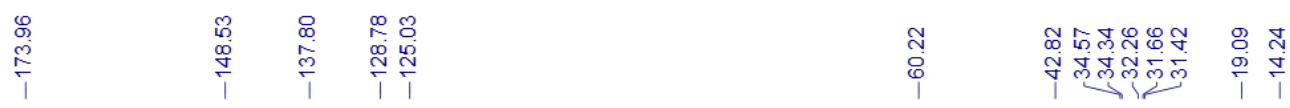<smiles>CCOC(=O)CCC(C)Cc1ccc(C(C)(C)C)cc1</smiles>

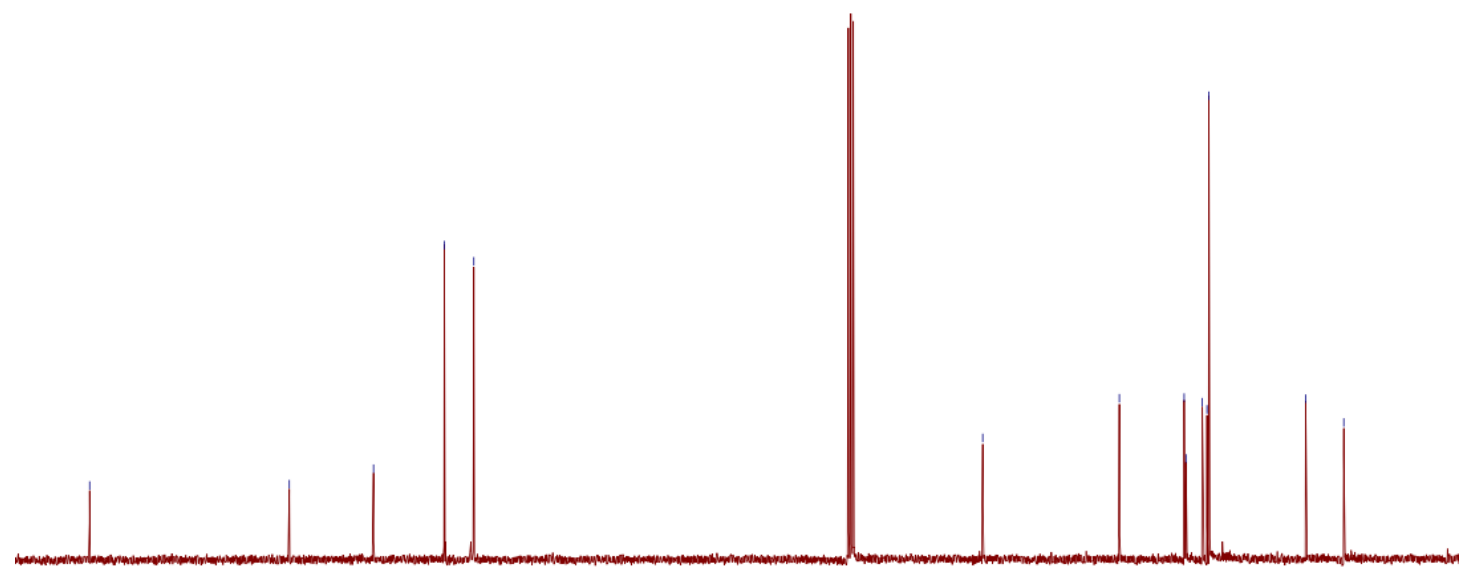

${ }_{180}^{1}$

$100 \quad \begin{gathered}90 \\ f 1\end{gathered}$ 
${ }^{1} \mathrm{H}$ NMR (400 MHz, $\left.\mathrm{CDCl}_{3}\right)$ of $\mathbf{3 h}$

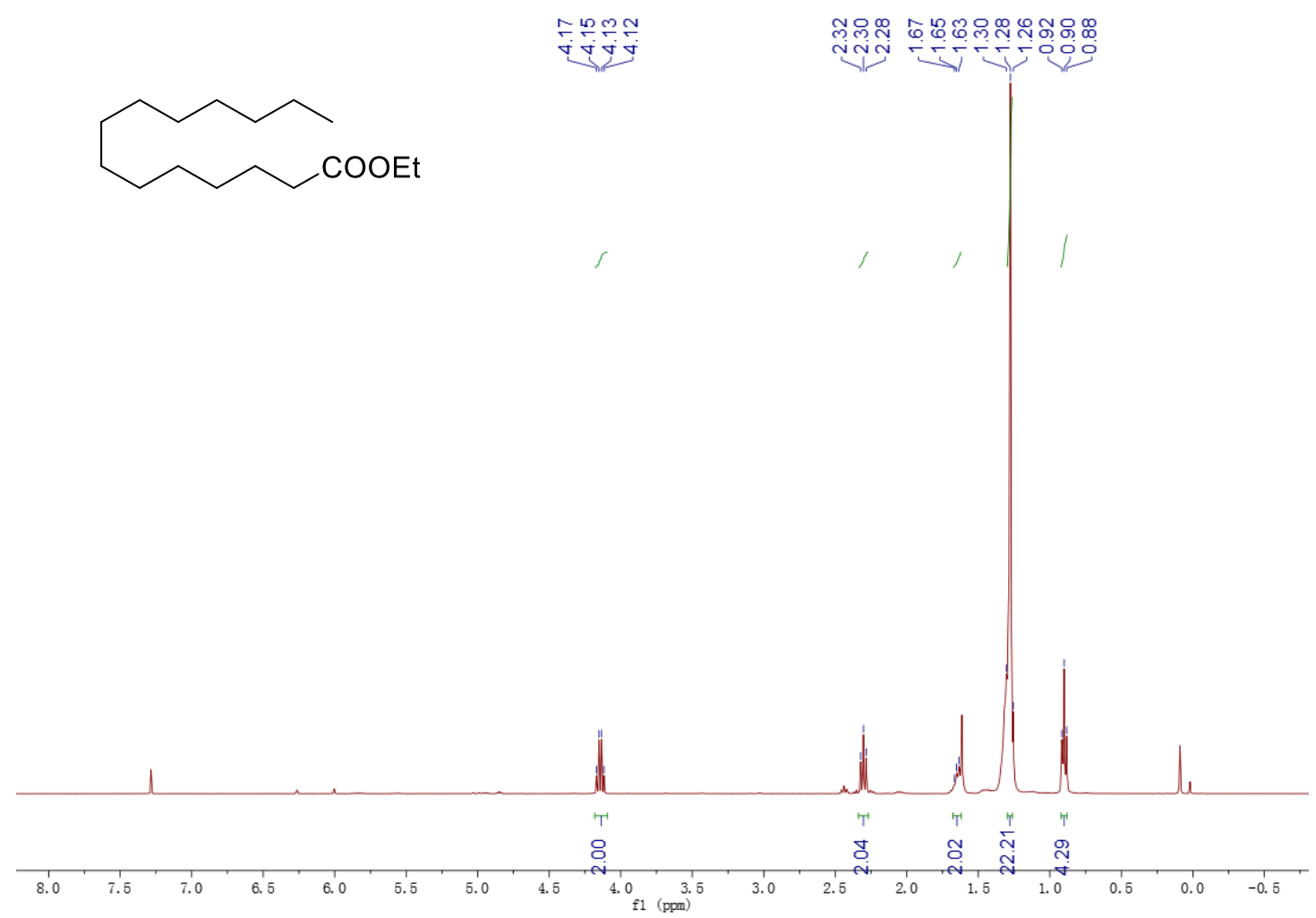

${ }^{13} \mathrm{C}\{1 \mathrm{H}\} \mathrm{NMR}\left(100 \mathrm{MHz}, \mathrm{CDCl}_{3}\right)$ of $\mathbf{3 h}$

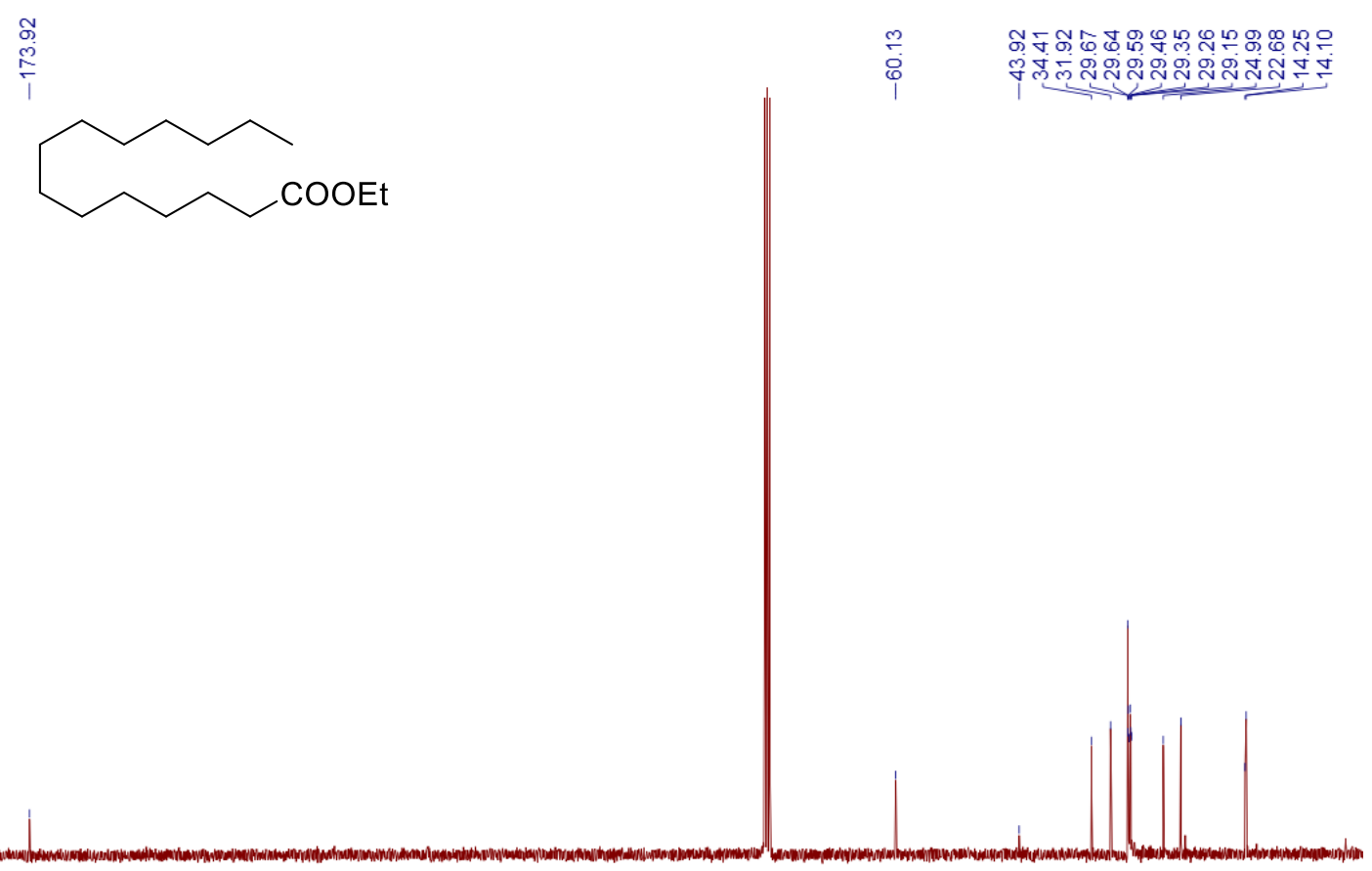

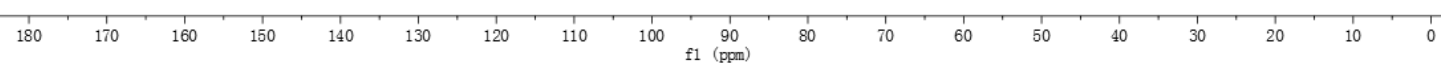


${ }^{1} \mathrm{H}$ NMR (400 MHz, $\mathrm{CDCl}_{3}$ ) of $\mathbf{3 i}$

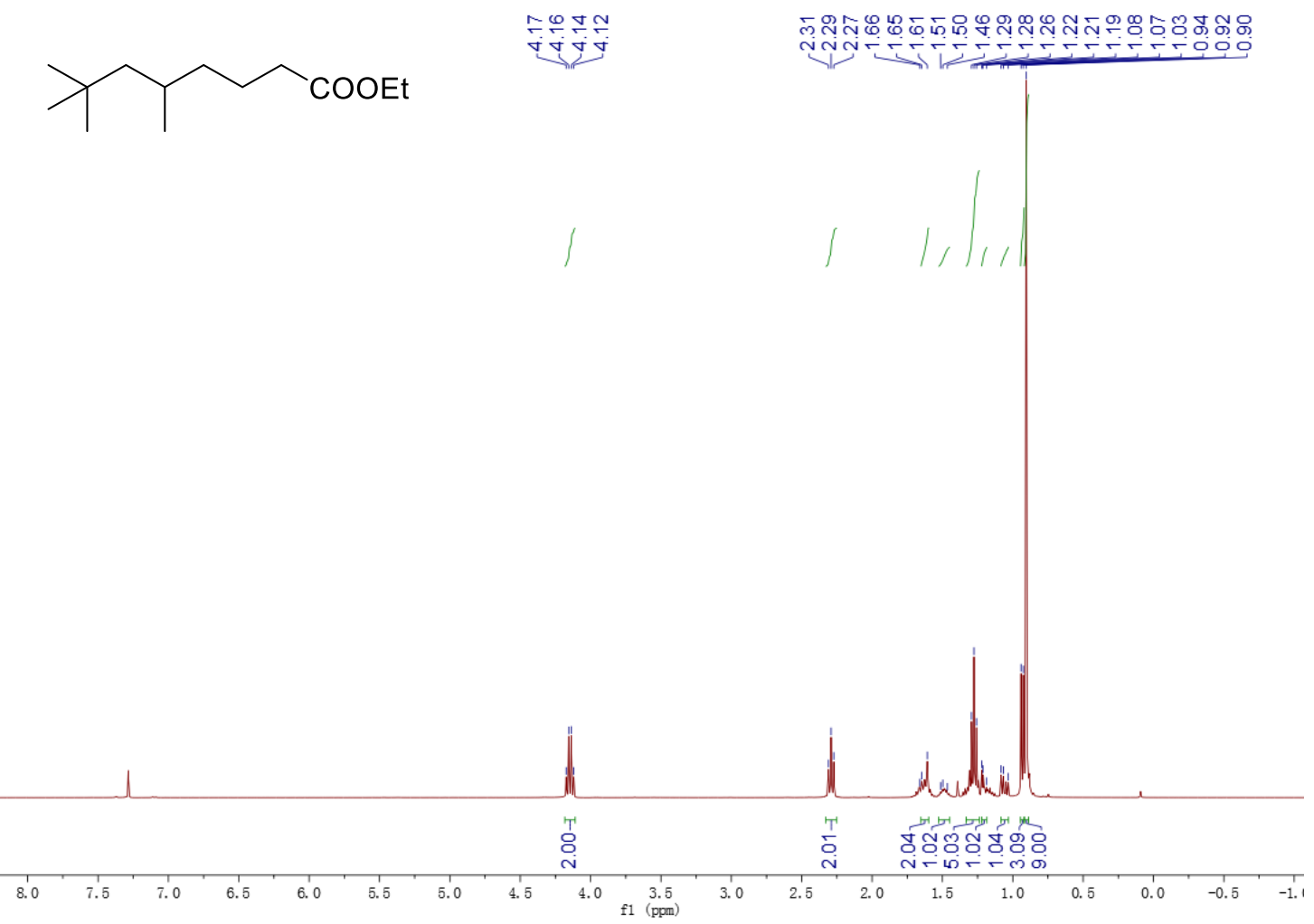

${ }^{13} \mathrm{C}\{1 \mathrm{H}\} \mathrm{NMR}\left(100 \mathrm{MHz}, \mathrm{CDCl}_{3}\right)$ of $\mathbf{3 i}$

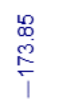

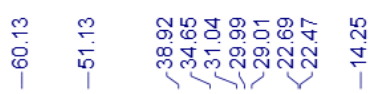<smiles>CCOC(=O)CCCC(C)CC(C)(C)C</smiles>
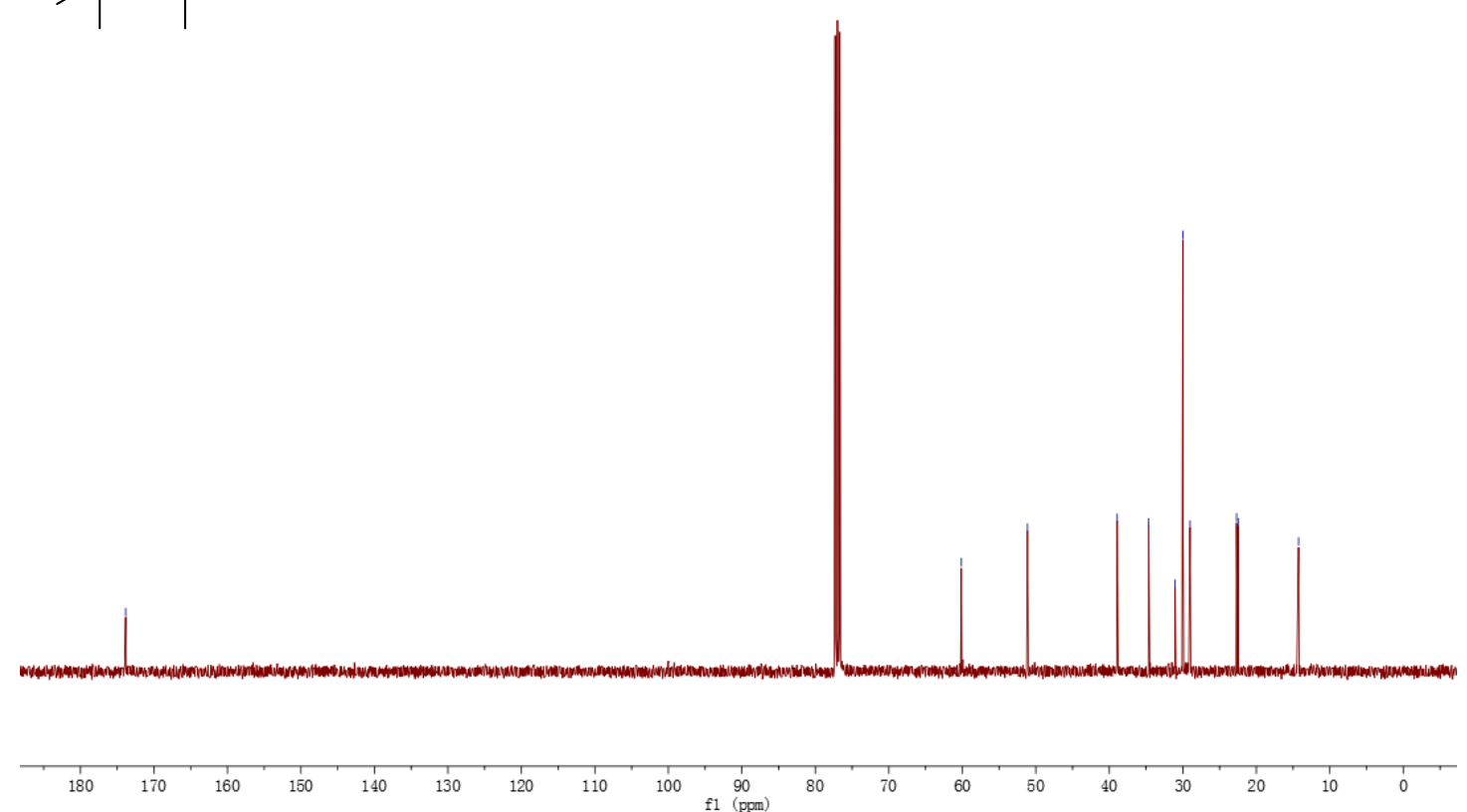
${ }^{1} \mathrm{H}$ NMR (400 MHz, $\left.\mathrm{CDCl}_{3}\right)$ of $\mathbf{3 j}$
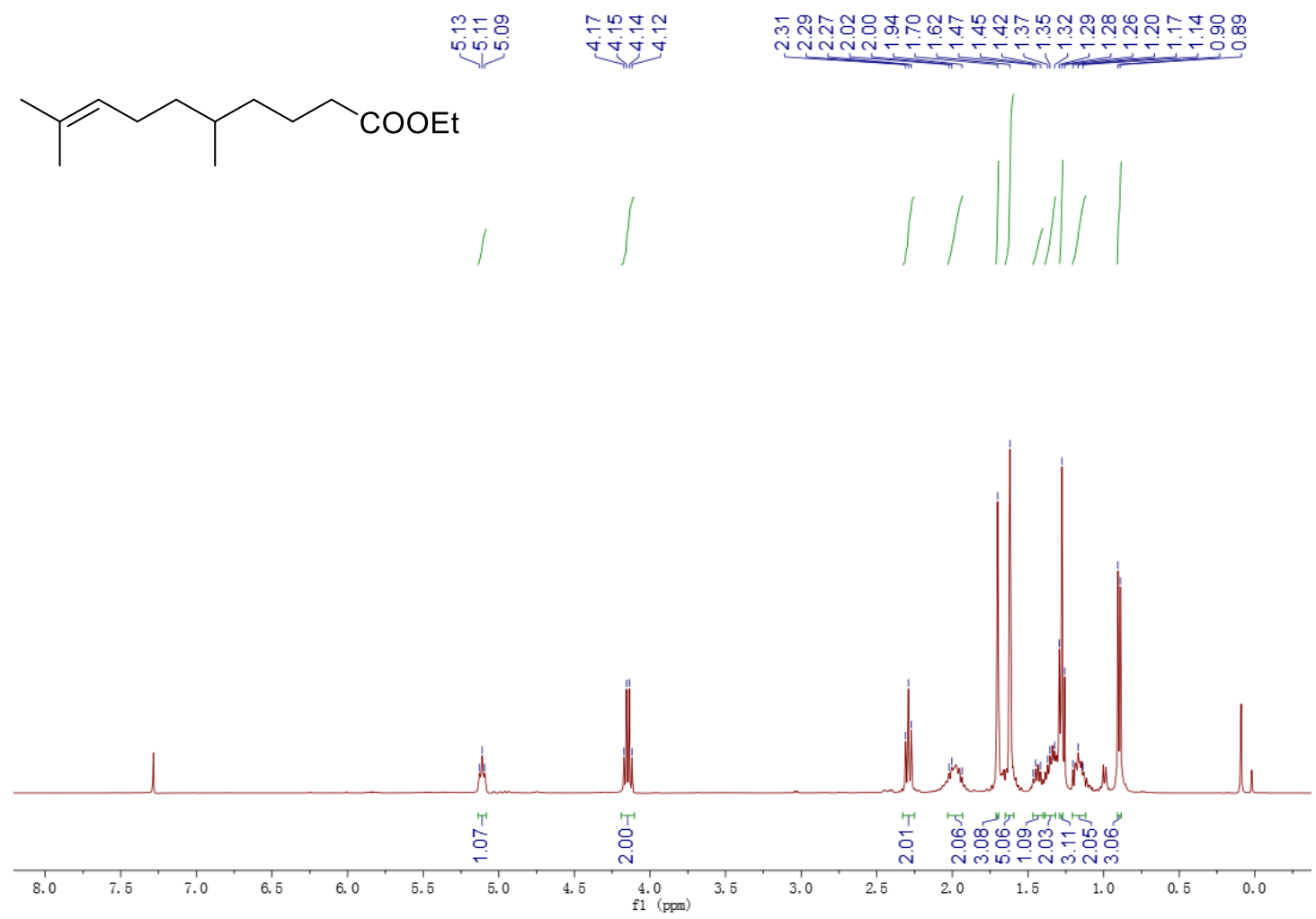

${ }^{13} \mathrm{C}\{1 \mathrm{H}\}$ NMR $\left(100 \mathrm{MHz}, \mathrm{CDCl}_{3}\right)$ of $\mathbf{3 j}$

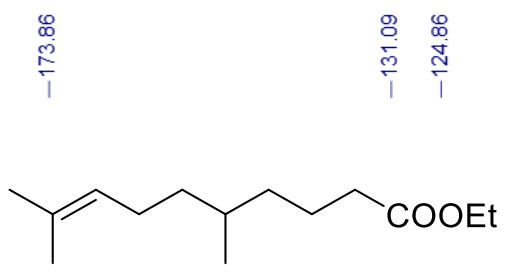

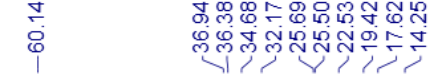
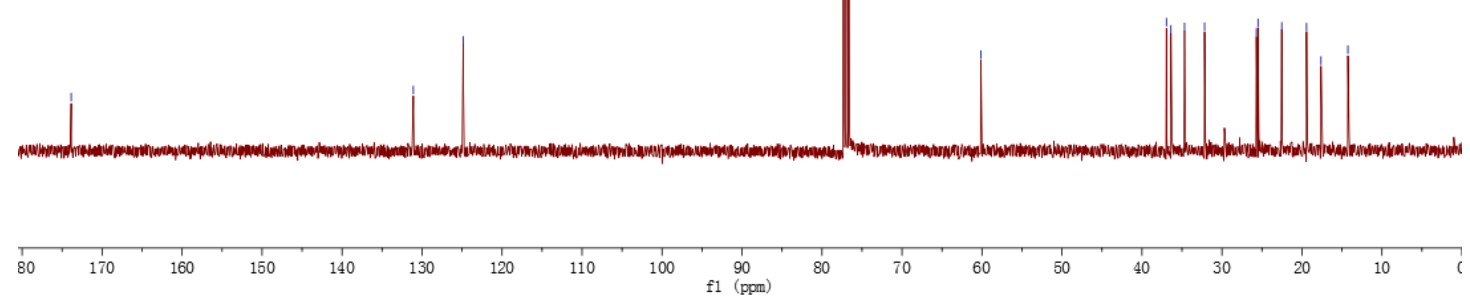
${ }^{1} \mathrm{H}$ NMR $\left(400 \mathrm{MHz}, \mathrm{CDCl}_{3}\right)$ of $\mathbf{3 k}$

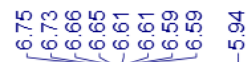

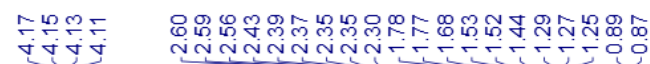

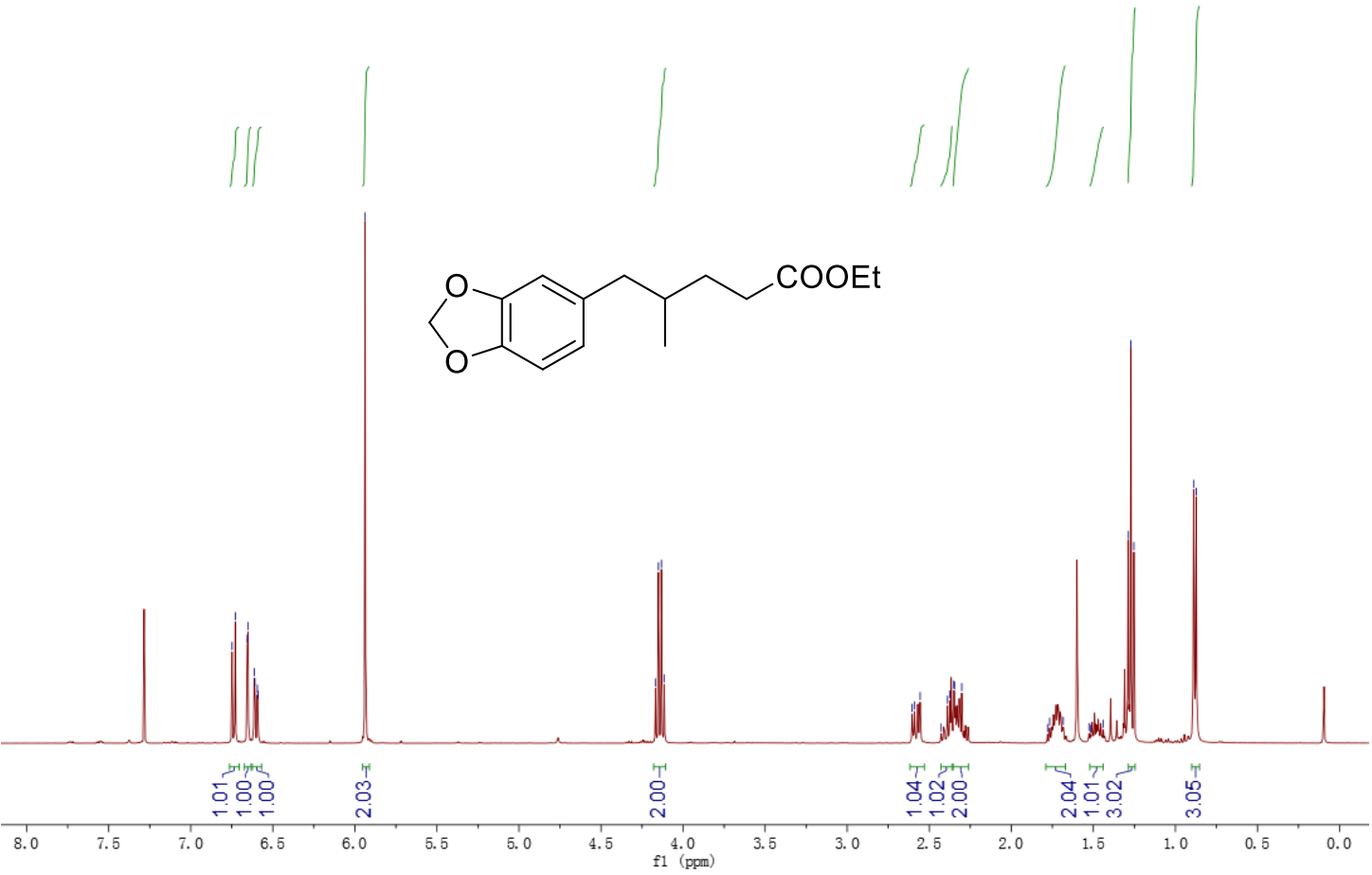

${ }^{13} \mathrm{C}\{1 \mathrm{H}\} \mathrm{NMR}\left(100 \mathrm{MHz}, \mathrm{CDCl}_{3}\right)$ of $\mathbf{3 k}$

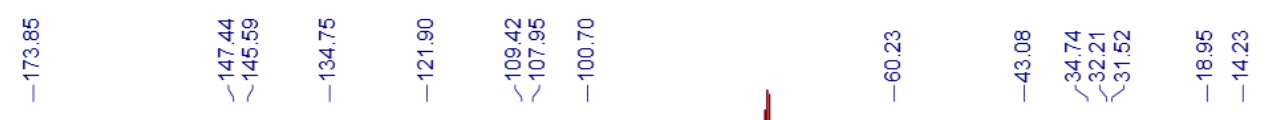<smiles>CCOC(=O)CCC(C)Cc1ccc2c(c1)OCO2</smiles>
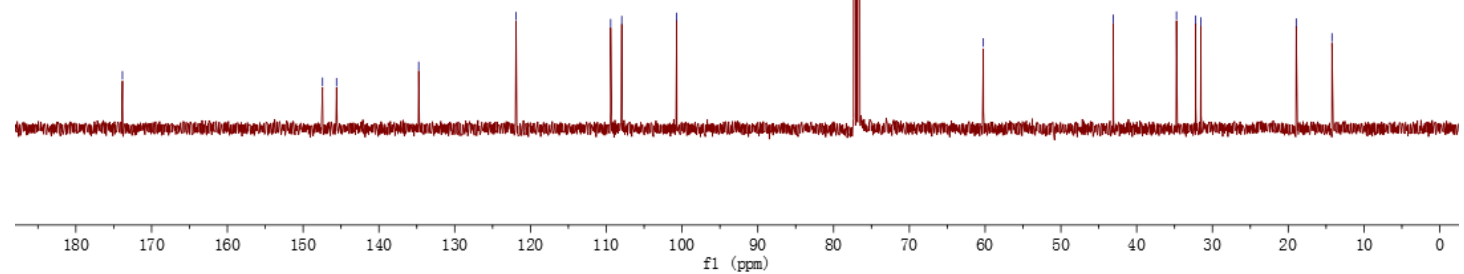
${ }^{1} \mathrm{H}$ NMR (400 MHz, $\mathrm{CDCl}_{3}$ ) of $\mathbf{3 I}$
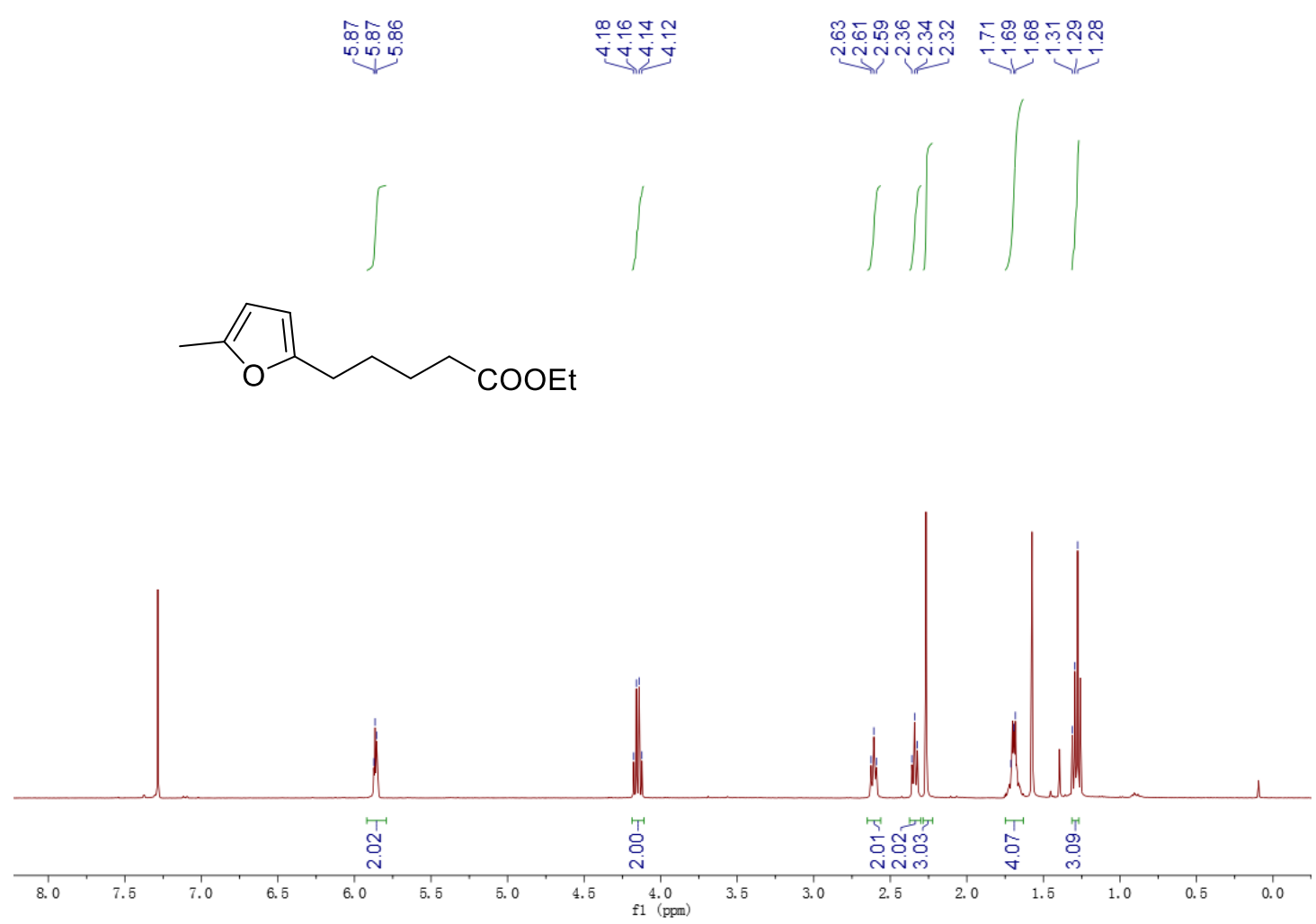

${ }^{13} \mathrm{C}\{1 \mathrm{H}\} \mathrm{NMR}\left(100 \mathrm{MHz}, \mathrm{CDCl}_{3}\right)$ of $3 \mathrm{I}$

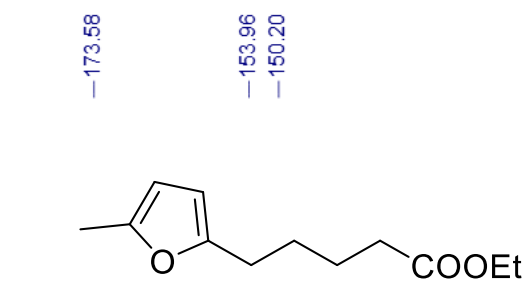

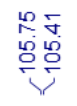

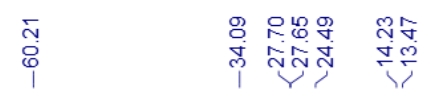

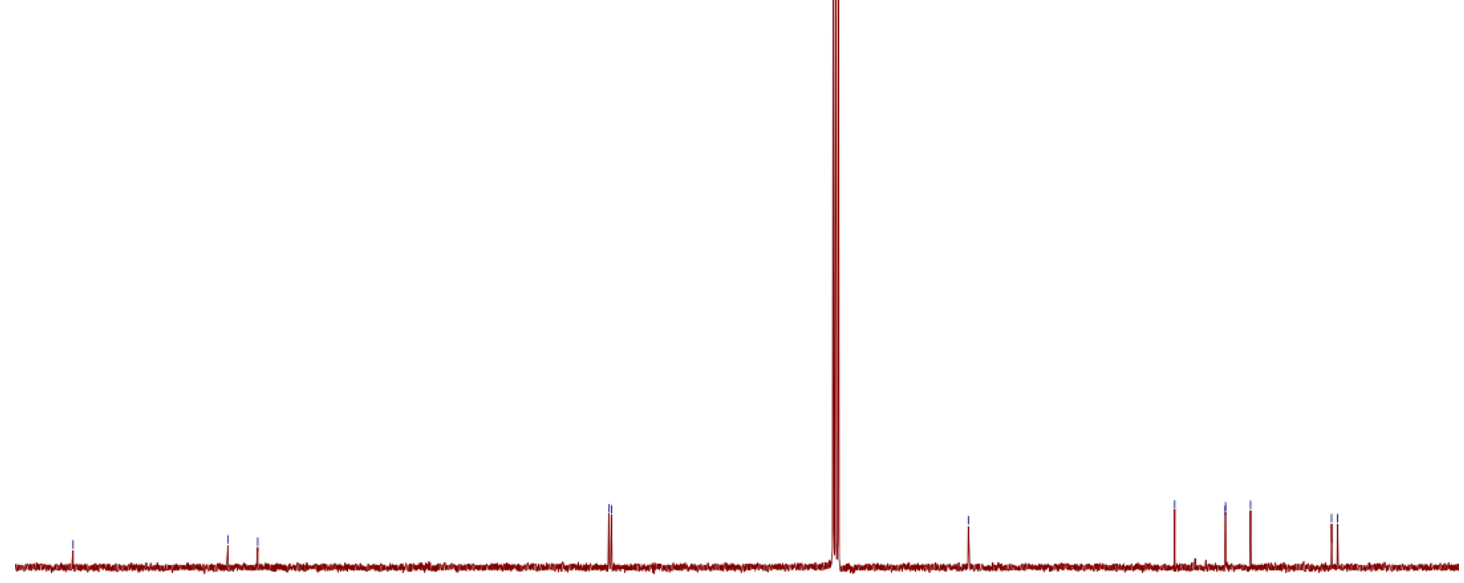

80

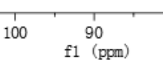


${ }^{1} \mathrm{H}$ NMR (400 MHz, $\left.\mathrm{CDCl}_{3}\right)$ of $\mathbf{3 m}$

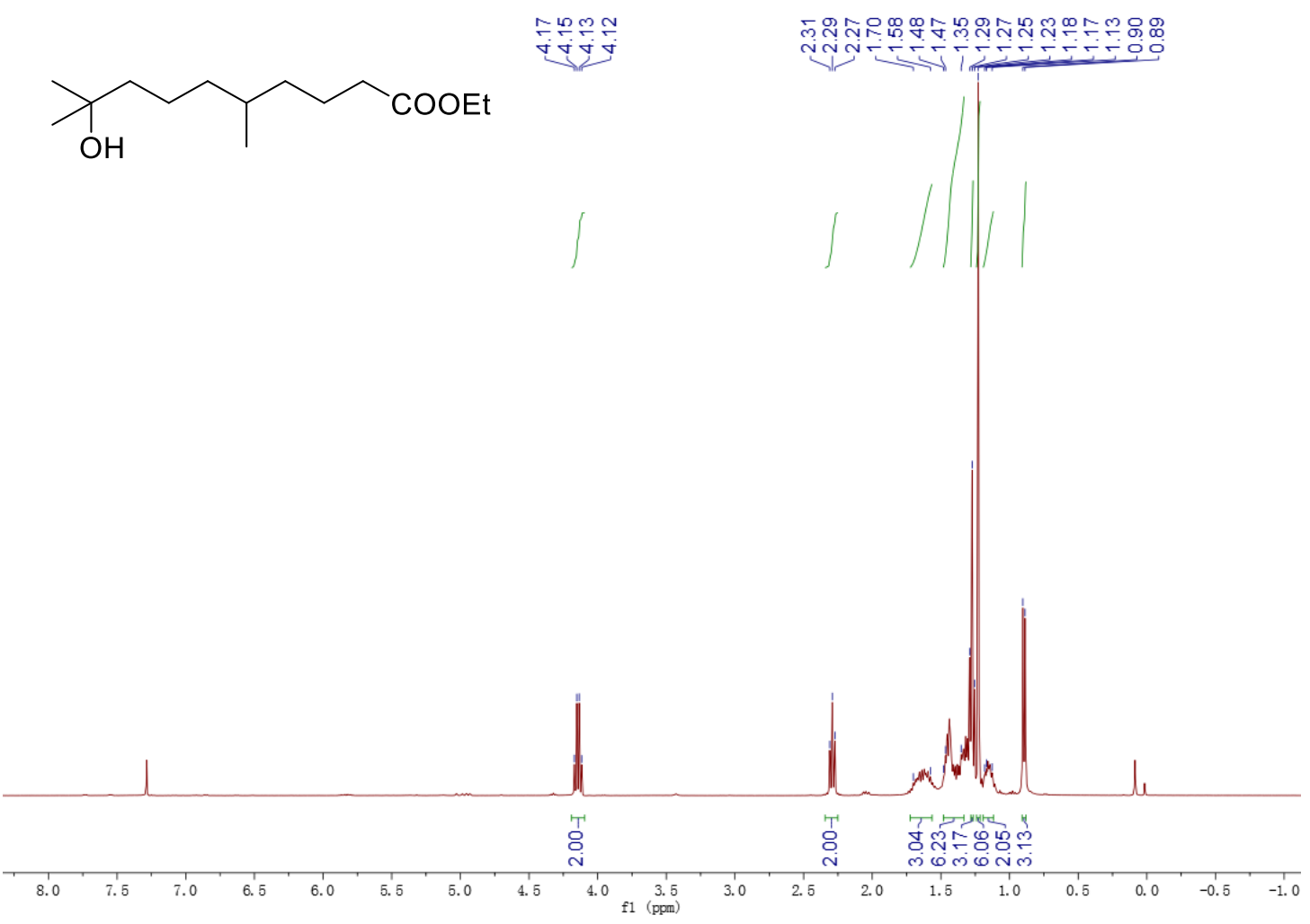

${ }^{13} \mathrm{C}\{1 \mathrm{H}\}$ NMR $\left(100 \mathrm{MHz}, \mathrm{CDCl}_{3}\right)$ of $\mathbf{3 m}$<smiles>CCOC(=O)CCCC(C)CCCC(C)(C)O</smiles>

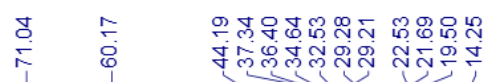

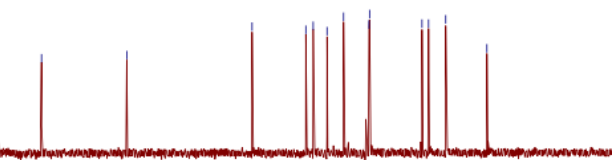

${ }_{180}^{1}$
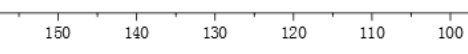

f1 90 
${ }^{1} \mathrm{H}$ NMR (400 MHz, $\left.\mathrm{CDCl}_{3}\right)$ of $\mathbf{3 n}$

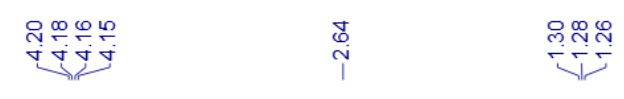

EtOOC $\sim$ COOEt

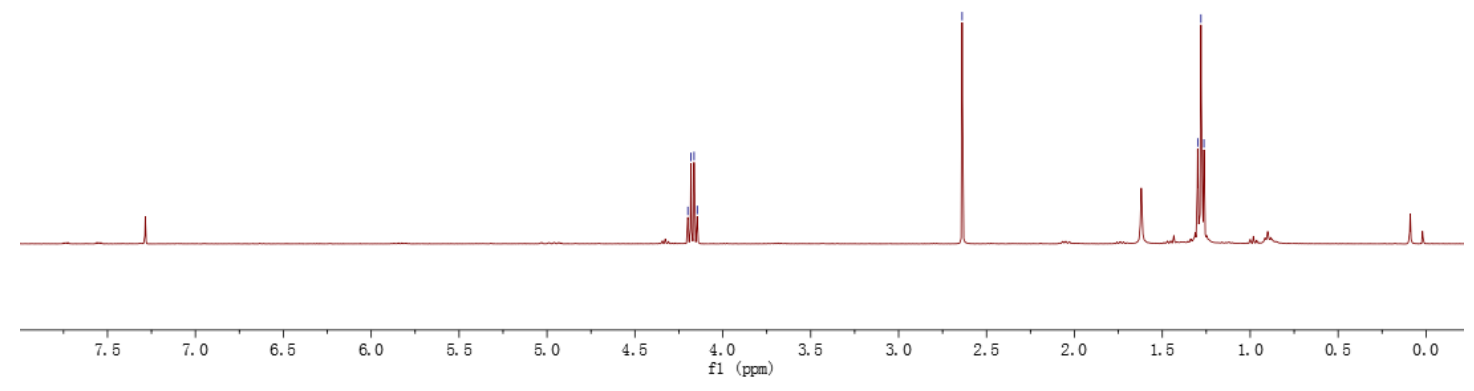

${ }^{13} \mathrm{C}\{1 \mathrm{H}\}$ NMR (100 MHz, $\left.\mathrm{CDCl}_{3}\right)$ of $\mathbf{3 n}$

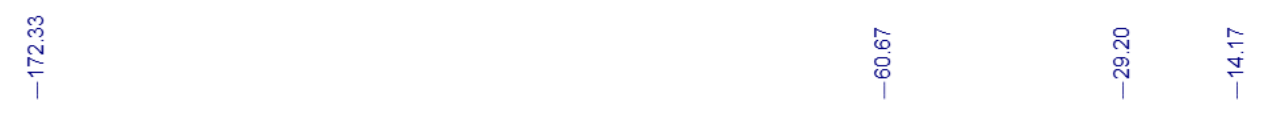

EtOOC $\sim \mathrm{COOEt}$

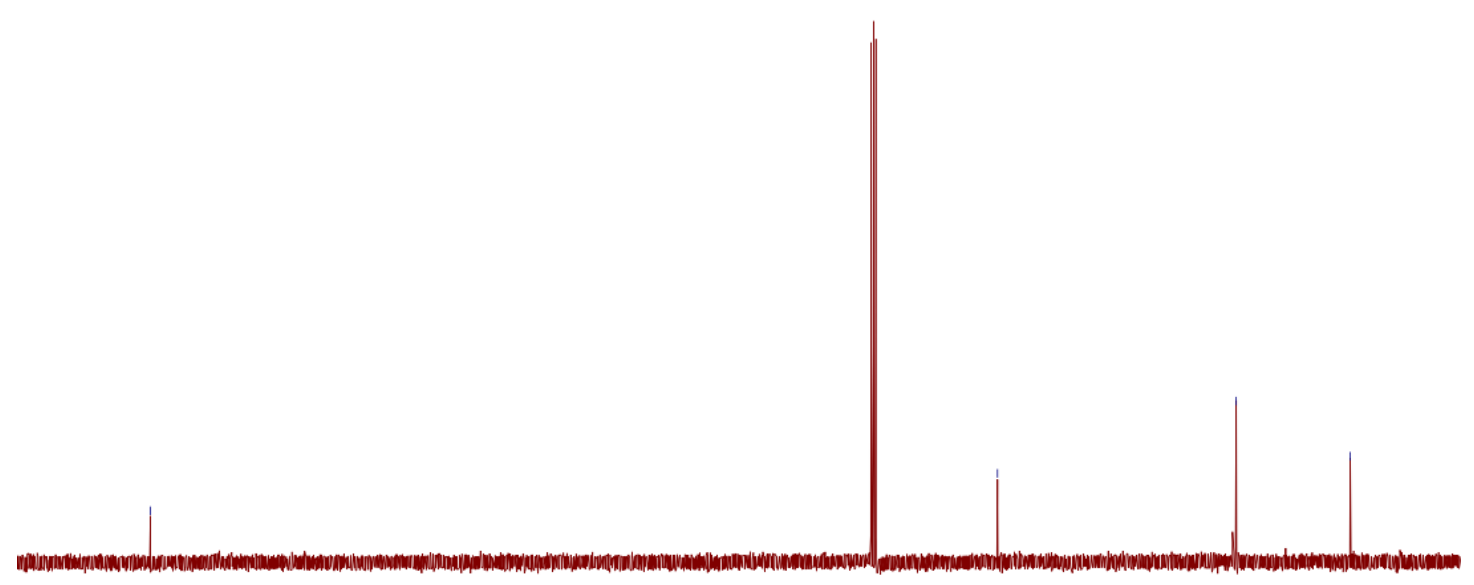

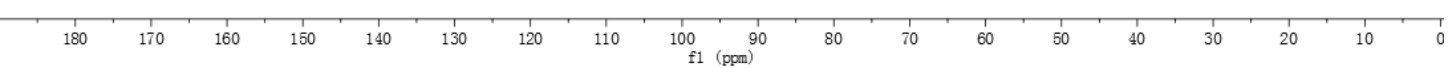


${ }^{1} \mathrm{H}$ NMR (400 MHz, $\left.\mathrm{CDCl}_{3}\right)$ of $\mathbf{3 o}$

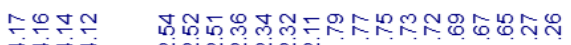

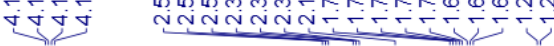

$\mathrm{S}_{\mathrm{COOEt}}$
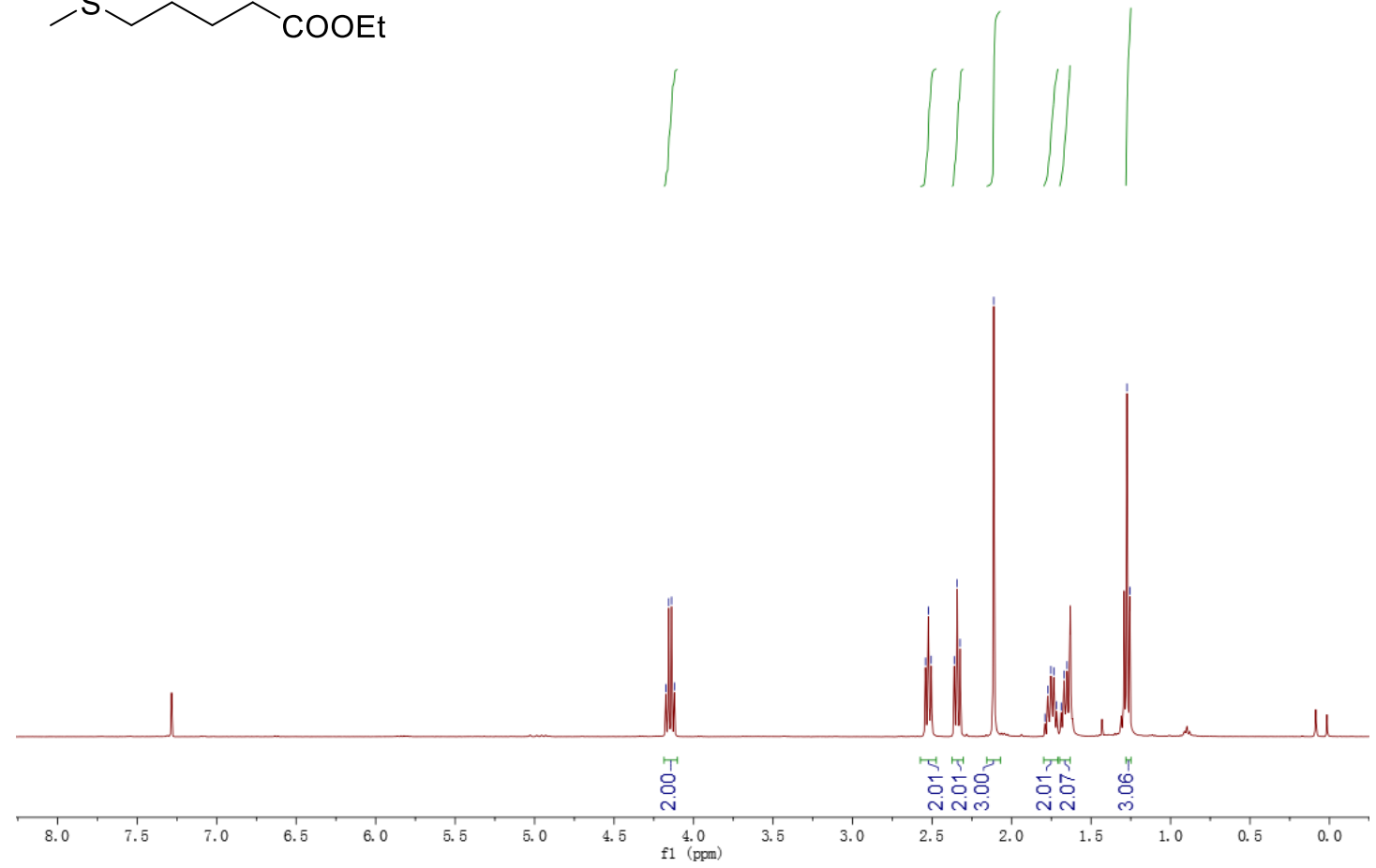

${ }^{13} \mathrm{C}\{1 \mathrm{H}\} \mathrm{NMR}\left(100 \mathrm{MHz}, \mathrm{CDCl}_{3}\right)$ of $\mathbf{3 o}$

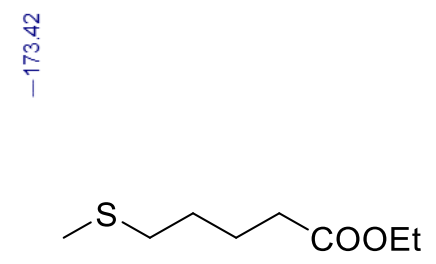

焉

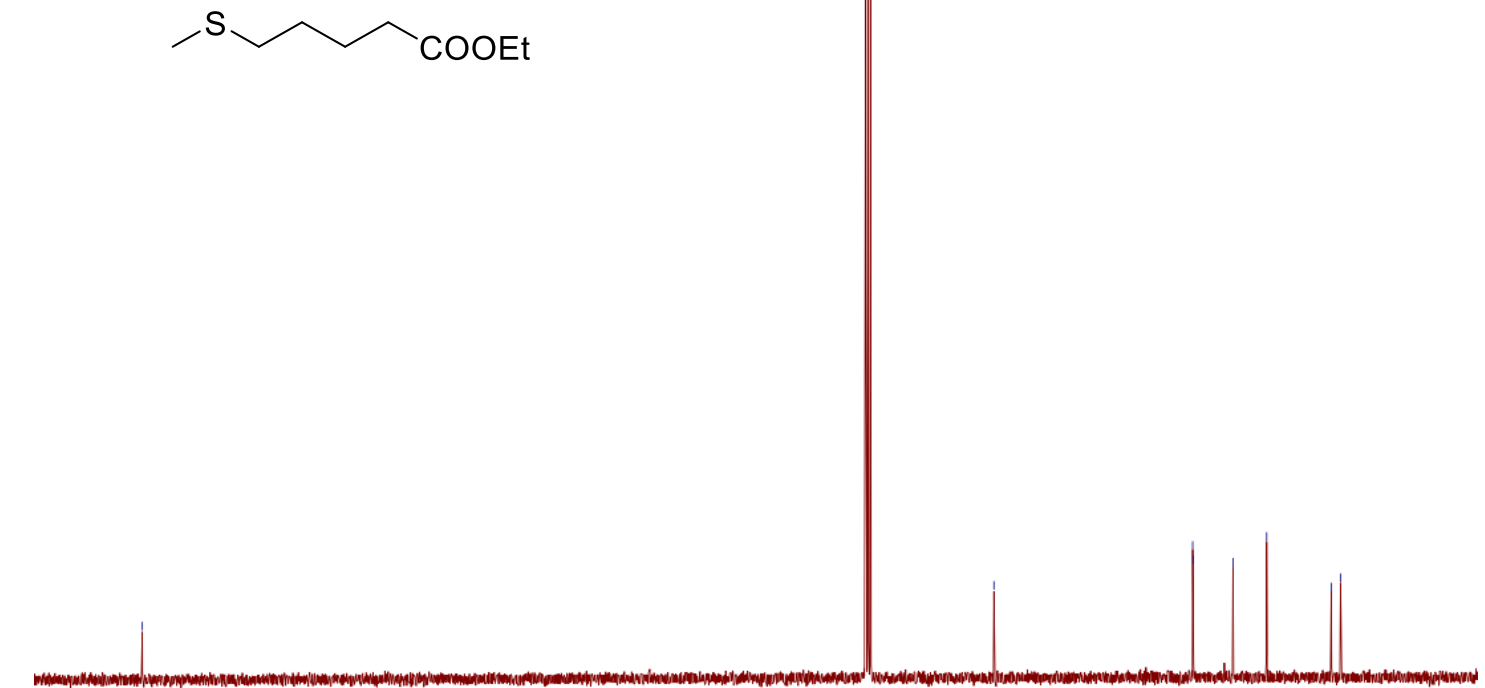

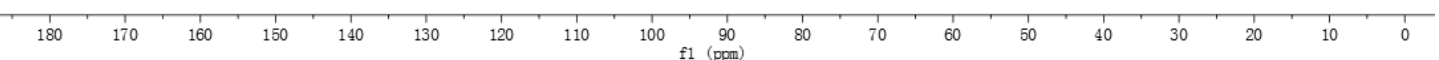


${ }^{1} \mathrm{H}$ NMR $\left(400 \mathrm{MHz}, \mathrm{CDCl}_{3}\right)$ of $\mathbf{3 p}$

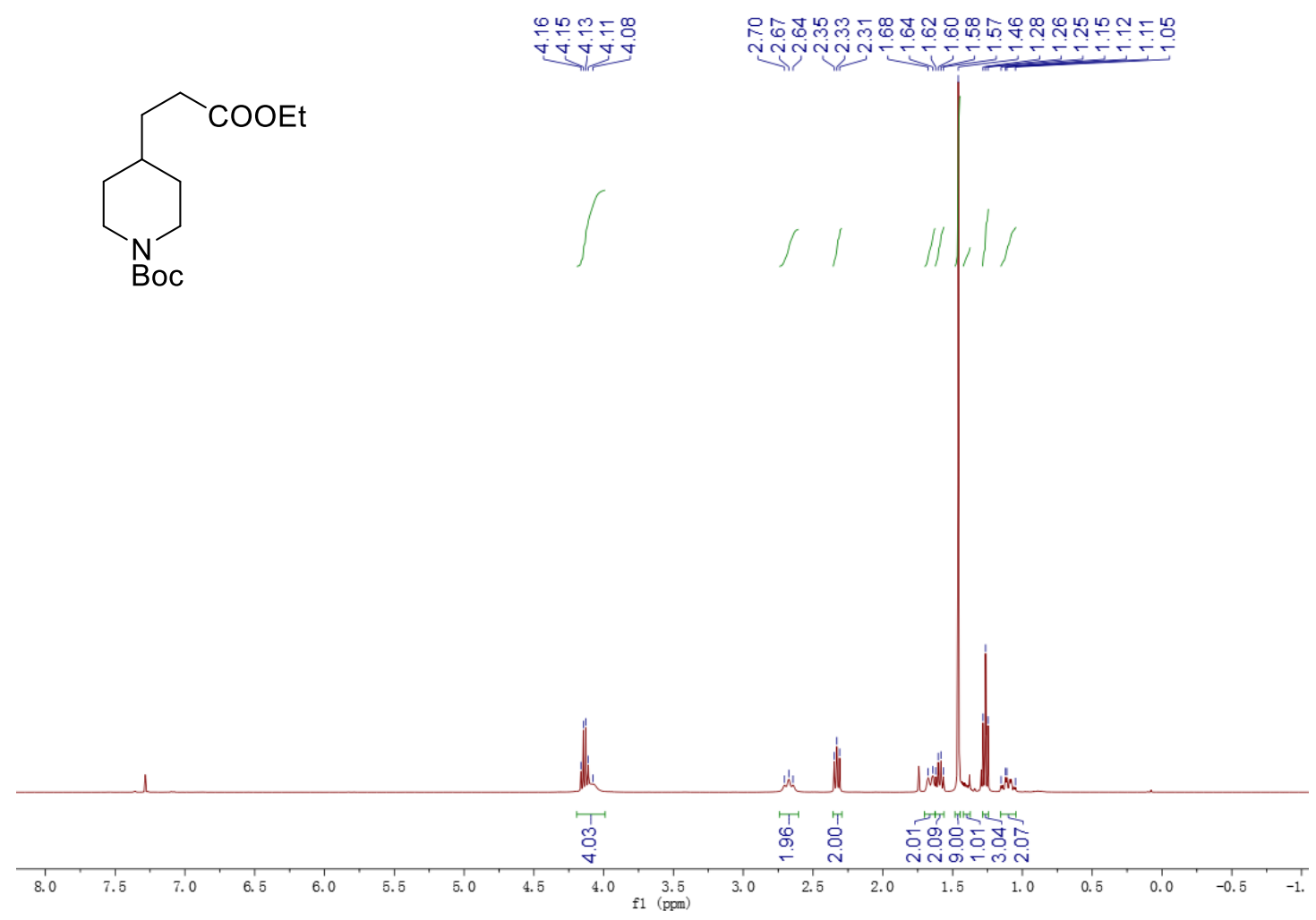

${ }^{13} \mathrm{C}\{1 \mathrm{H}\} \mathrm{NMR}\left(100 \mathrm{MHz}, \mathrm{CDCl}_{3}\right)$ of $\mathbf{3 p}$

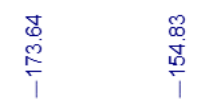

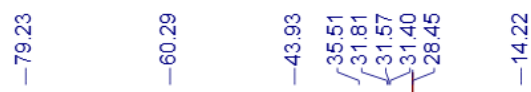<smiles>CCOC(=O)CCC1CCN(C(=O)OCc2ccccc2)CC1</smiles>
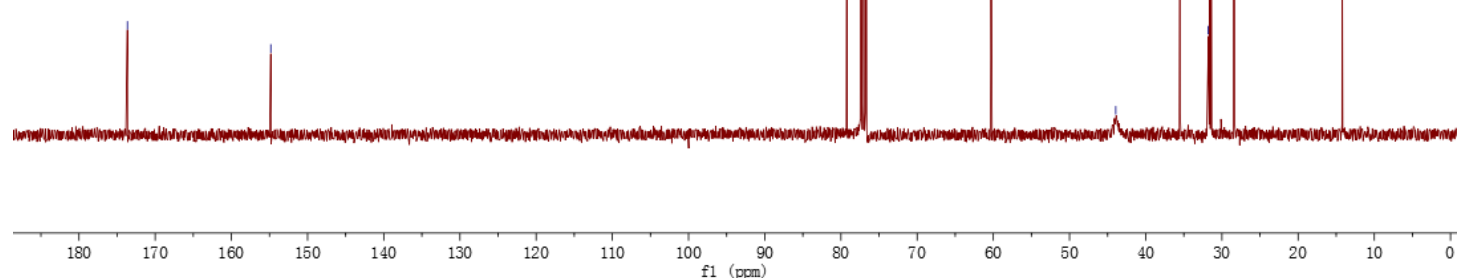
${ }^{1} \mathrm{H}$ NMR (400 MHz, $\left.\mathrm{CDCl}_{3}\right)$ of $\mathbf{3 q}$

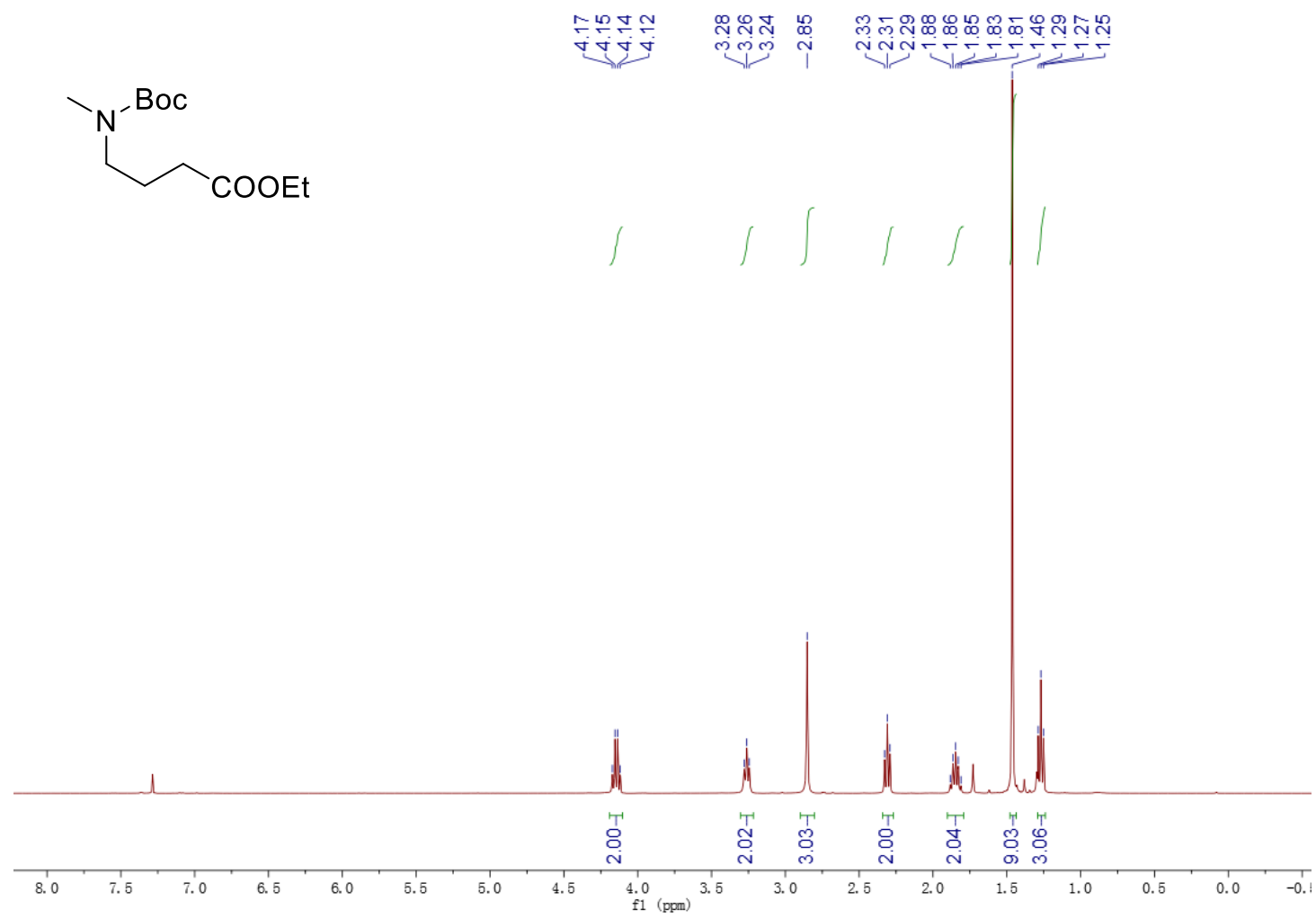

${ }^{13} \mathrm{C}\{1 \mathrm{H}\} \mathrm{NMR}\left(100 \mathrm{MHz}, \mathrm{CDCl}_{3}\right)$ of $\mathbf{3 q}$
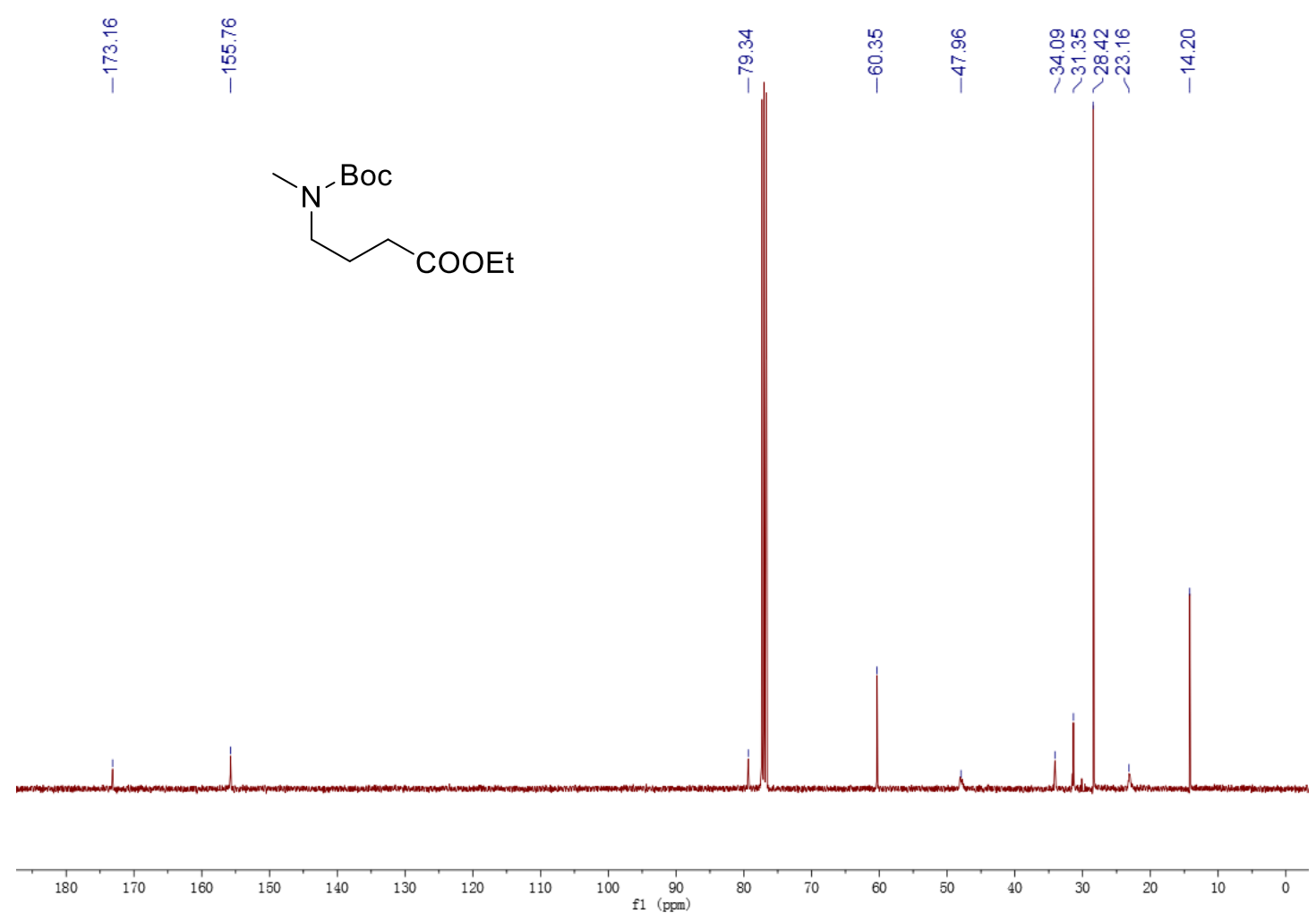
${ }^{1} \mathrm{H}$ NMR (400 MHz, $\mathrm{CDCl}_{3}$ ) of $\mathbf{3 r}$

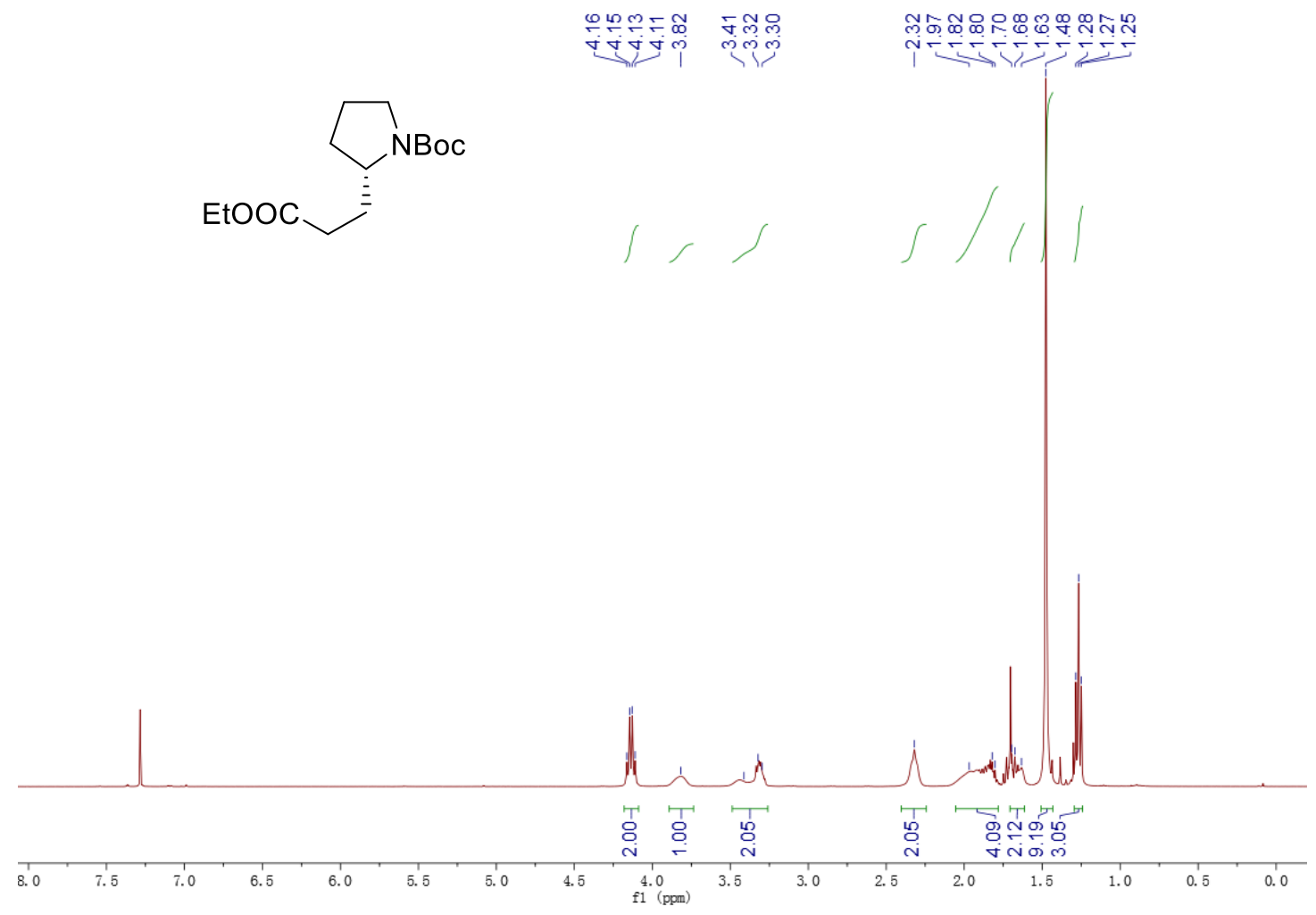

${ }^{13} \mathrm{C}\{1 \mathrm{H}\}$ NMR $\left(100 \mathrm{MHz}, \mathrm{CDCl}_{3}\right)$ of $\mathbf{3 r}$
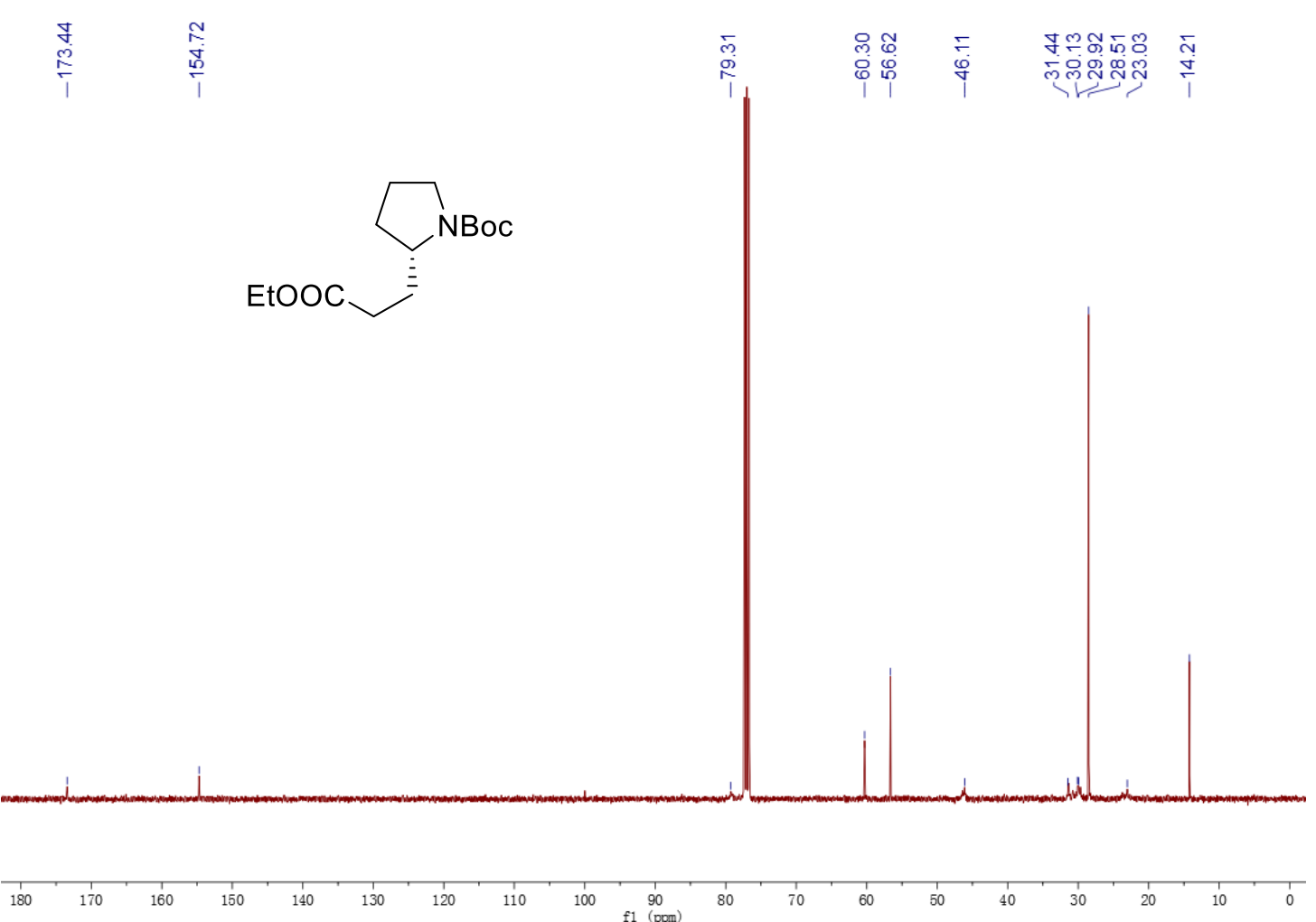
${ }^{1} \mathrm{H}$ NMR (400 MHz, $\mathrm{CDCl}_{3}$ ) of $\mathbf{3 s}$

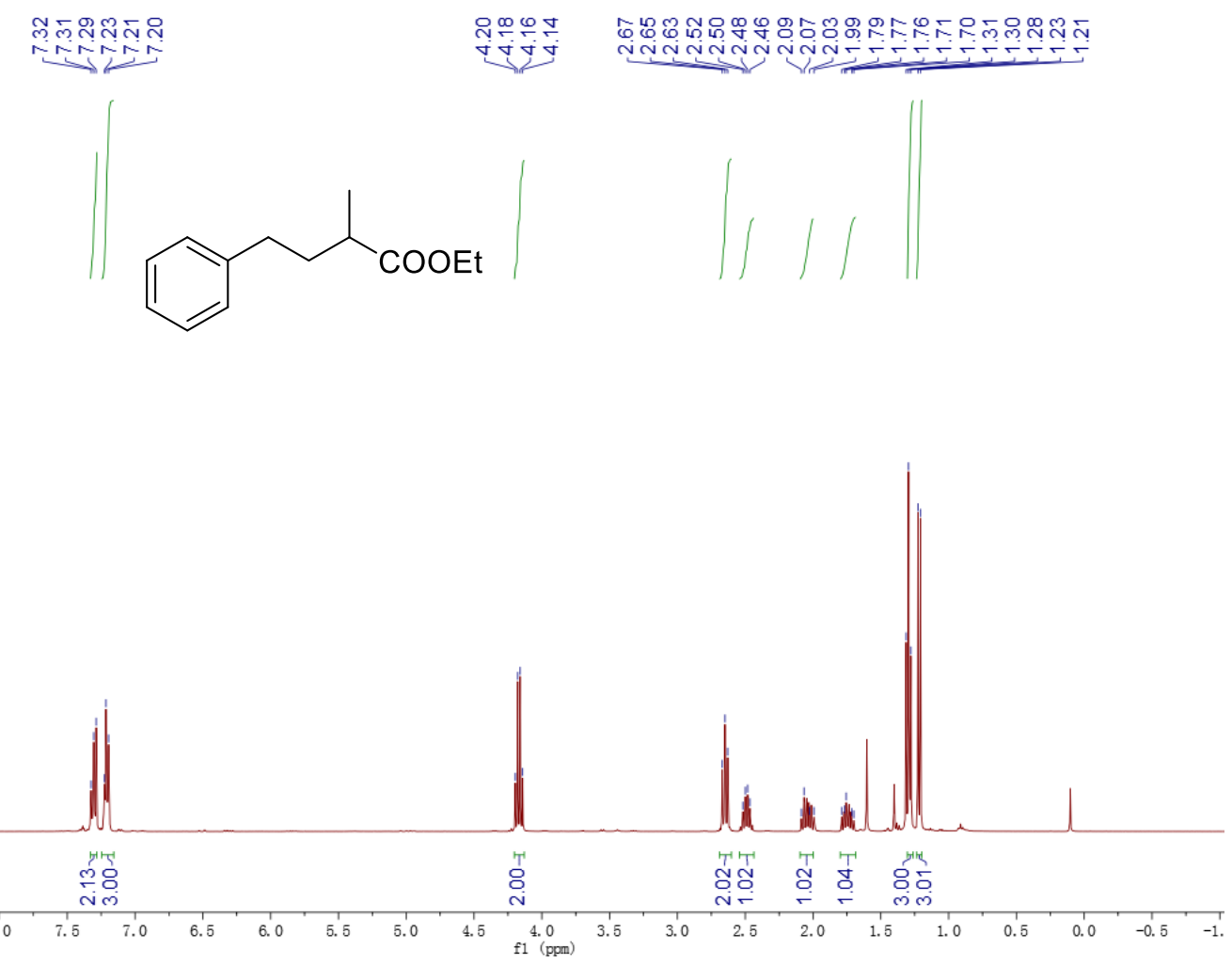

${ }^{13} \mathrm{C}\{1 \mathrm{H}\} \mathrm{NMR}\left(100 \mathrm{MHz}, \mathrm{CDCl}_{3}\right)$ of $\mathbf{3 s}$

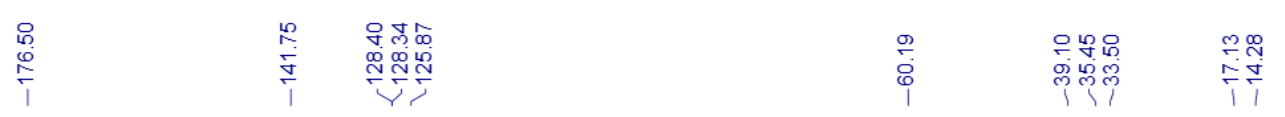<smiles>CCOC(=O)C(C)CCc1ccccc1</smiles>

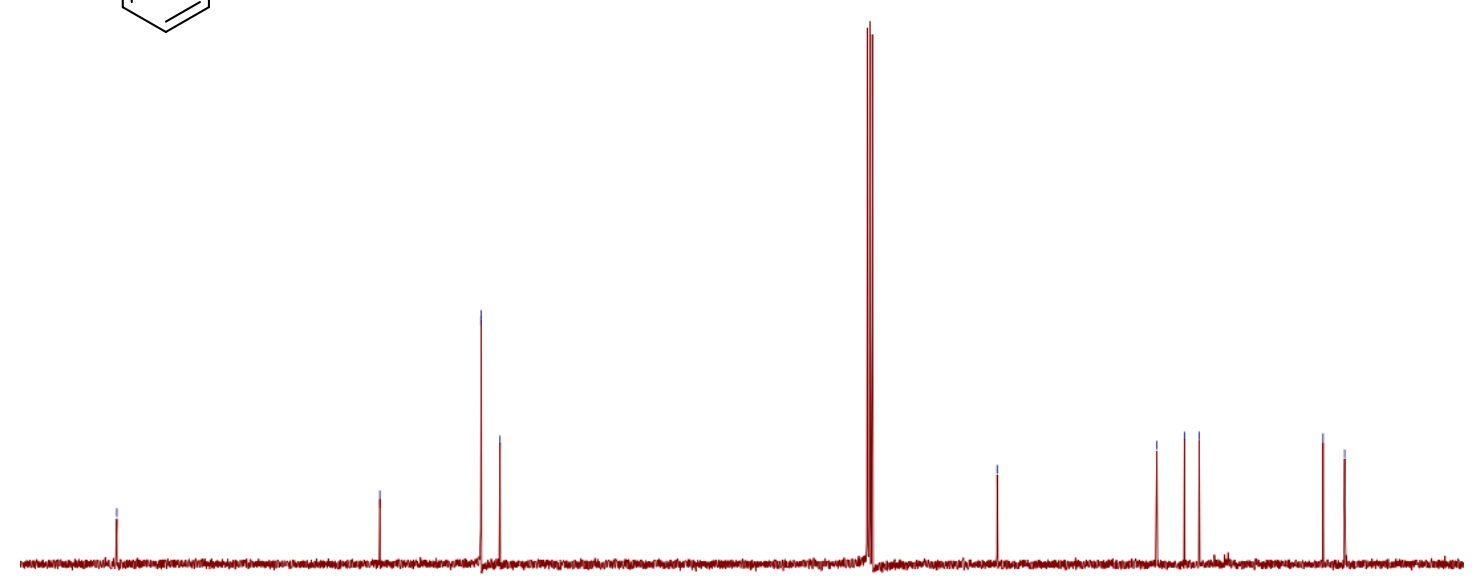

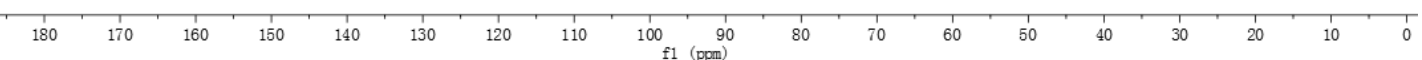


${ }^{1} \mathrm{H}$ NMR (400 MHz, $\mathrm{CDCl}_{3}$ ) of $\mathbf{3 t}$

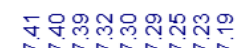

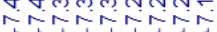

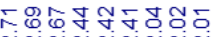

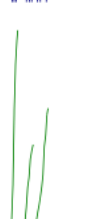<smiles>C1=CC=C1</smiles>

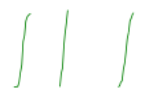<smiles>O=C(CCCc1ccccc1)OCc1ccccc1</smiles>

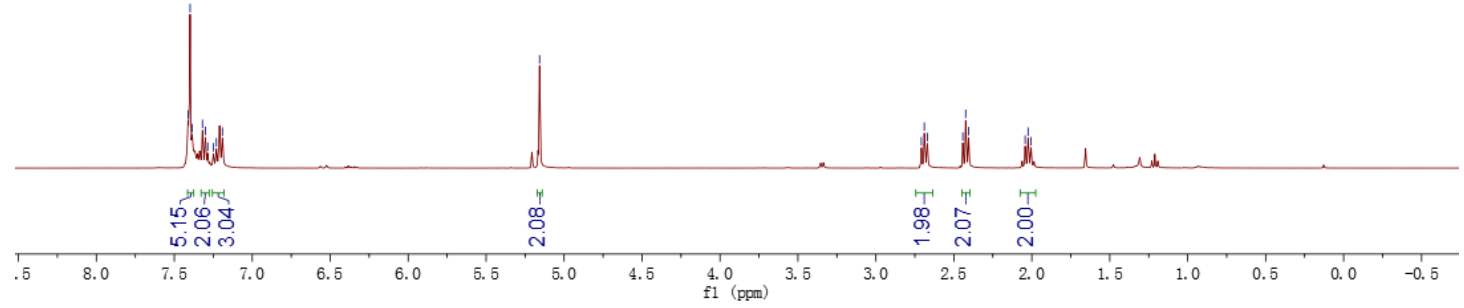

${ }^{13} \mathrm{C}\{1 \mathrm{H}\} \mathrm{NMR}\left(100 \mathrm{MHz}, \mathrm{CDCl}_{3}\right)$ of $\mathbf{3 t}$
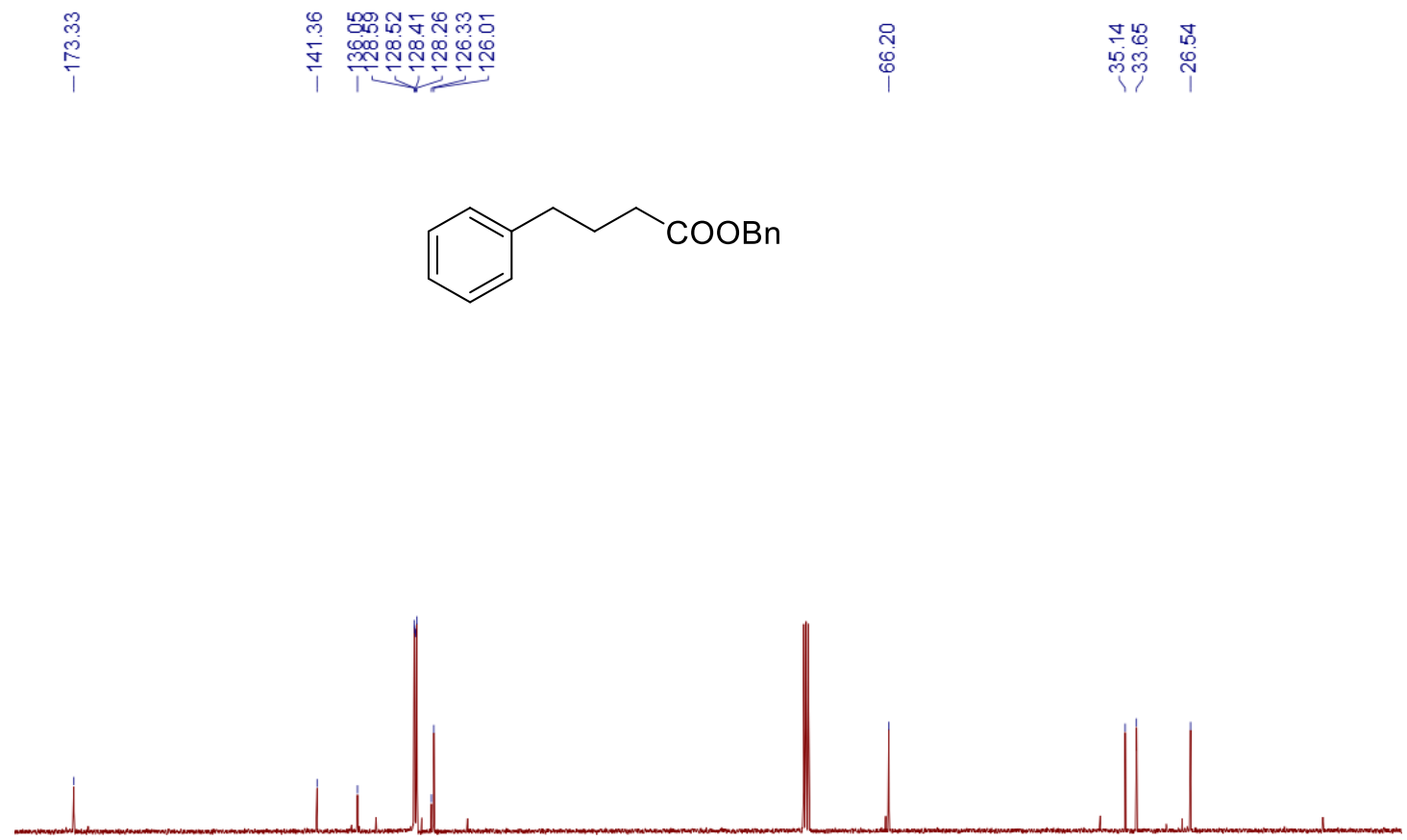

$\stackrel{T}{180}$
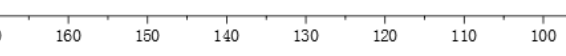

$\stackrel{90}{90}$ 
${ }^{1} \mathrm{H}$ NMR (400 MHz, $\mathrm{CDCl}_{3}$ ) of $\mathbf{5 a}$

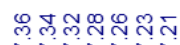

MTnNan

ஹ

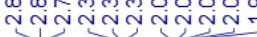

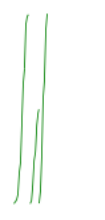<smiles>N#CCCCc1ccccc1</smiles>
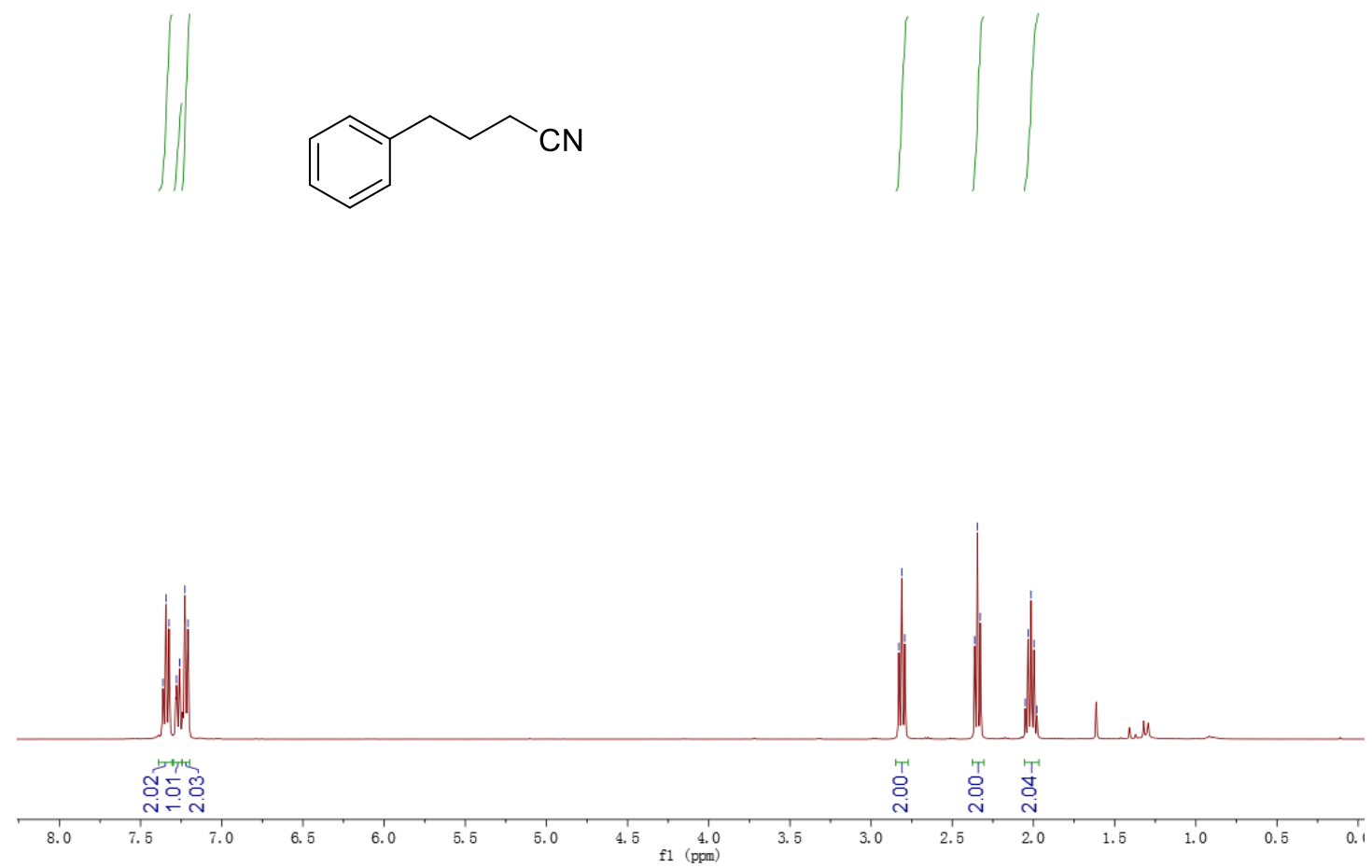

${ }^{13} \mathrm{C}\{1 \mathrm{H}\} \mathrm{NMR}\left(100 \mathrm{MHz}, \mathrm{CDCl}_{3}\right)$ of $\mathbf{5 a}$

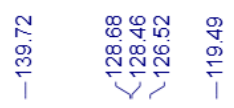

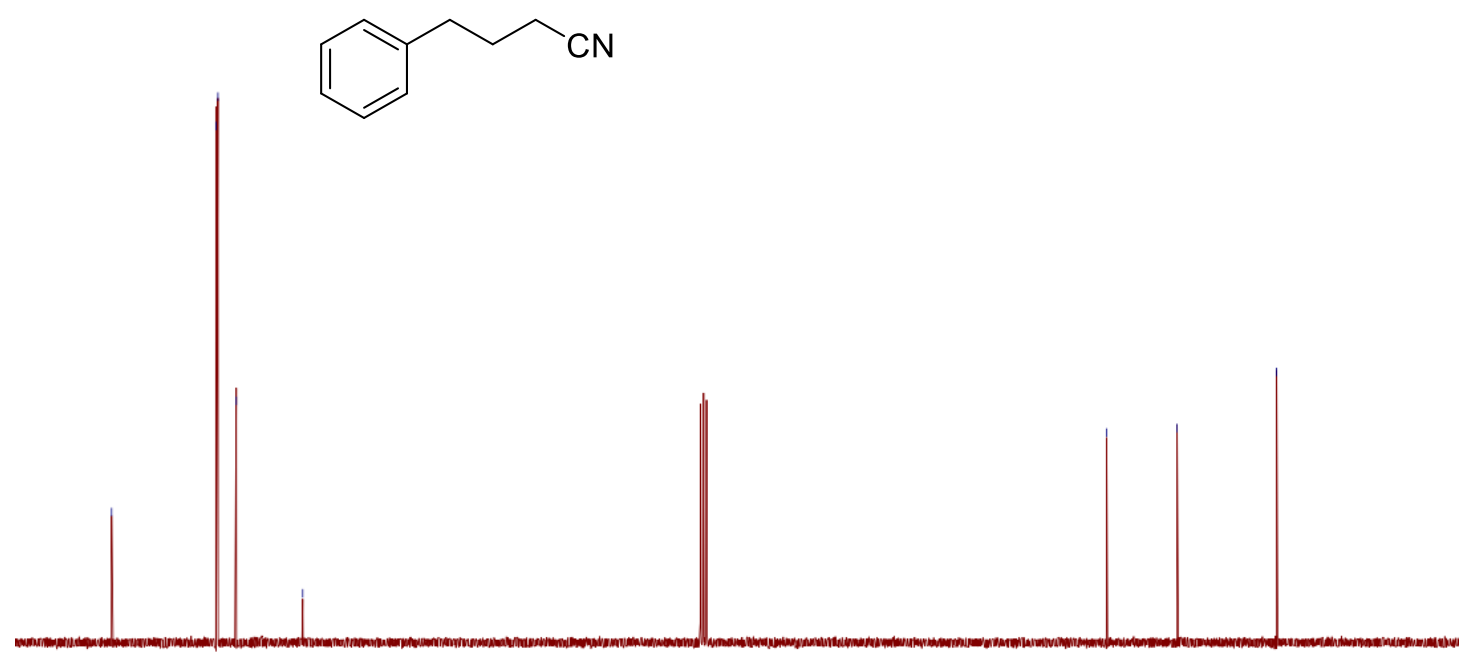

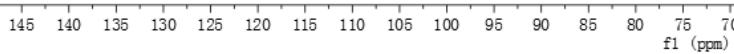


${ }^{1} \mathrm{H}$ NMR (400 MHz, $\left.\mathrm{CDCl}_{3}\right)$ of $\mathbf{5 b}$

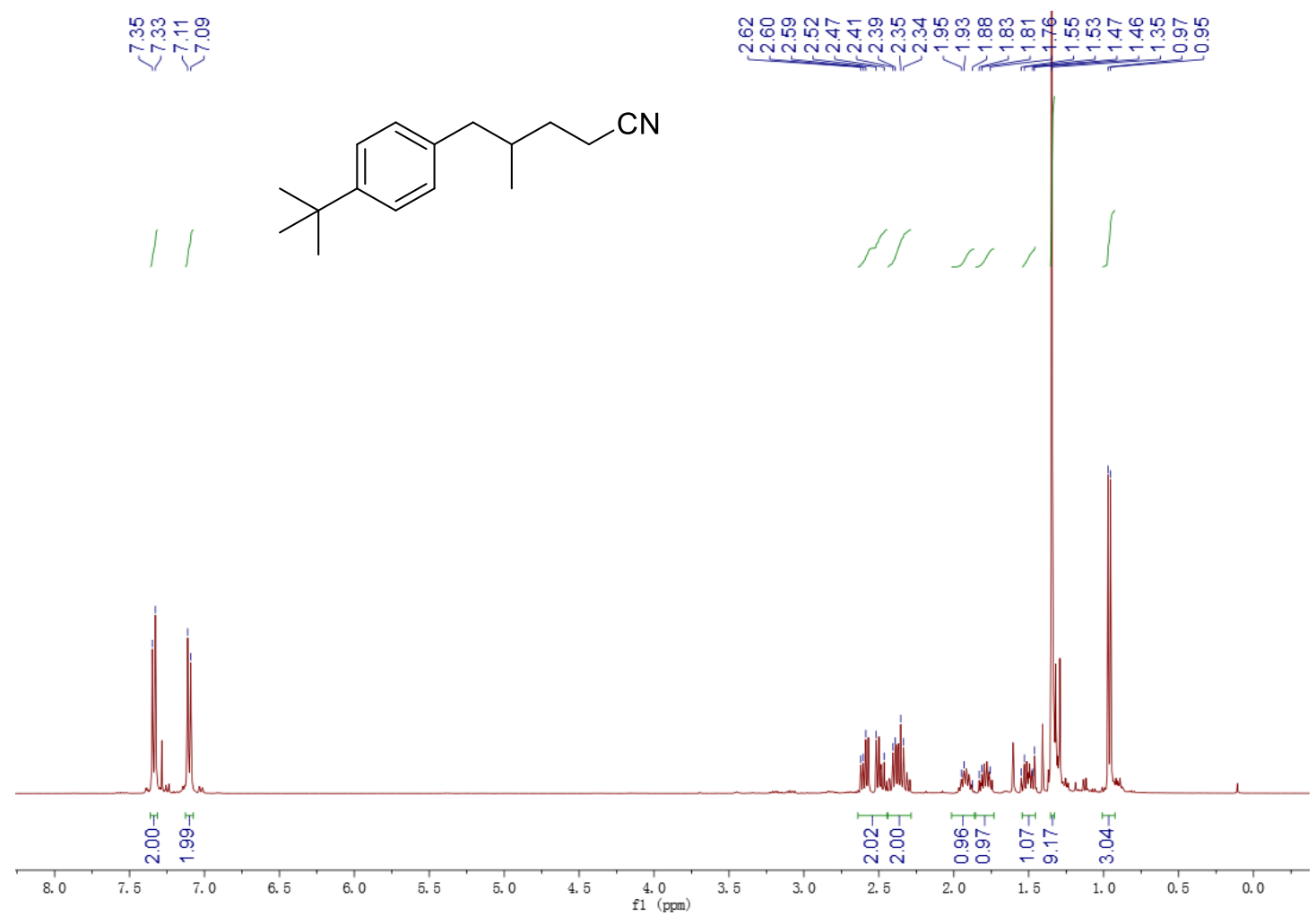

${ }^{13} \mathrm{C}\{1 \mathrm{H}\} \mathrm{NMR}\left(100 \mathrm{MHz}, \mathrm{CDCl}_{3}\right)$ of $\mathbf{5 b}$

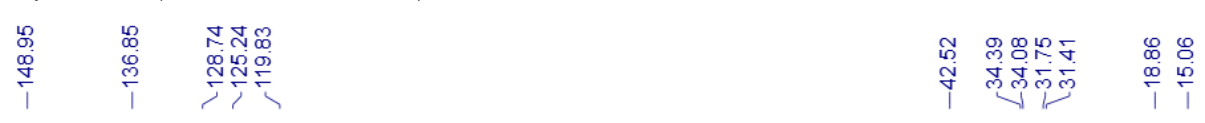<smiles>CC(CCC#N)Cc1ccc(C(C)(C)C)cc1</smiles>
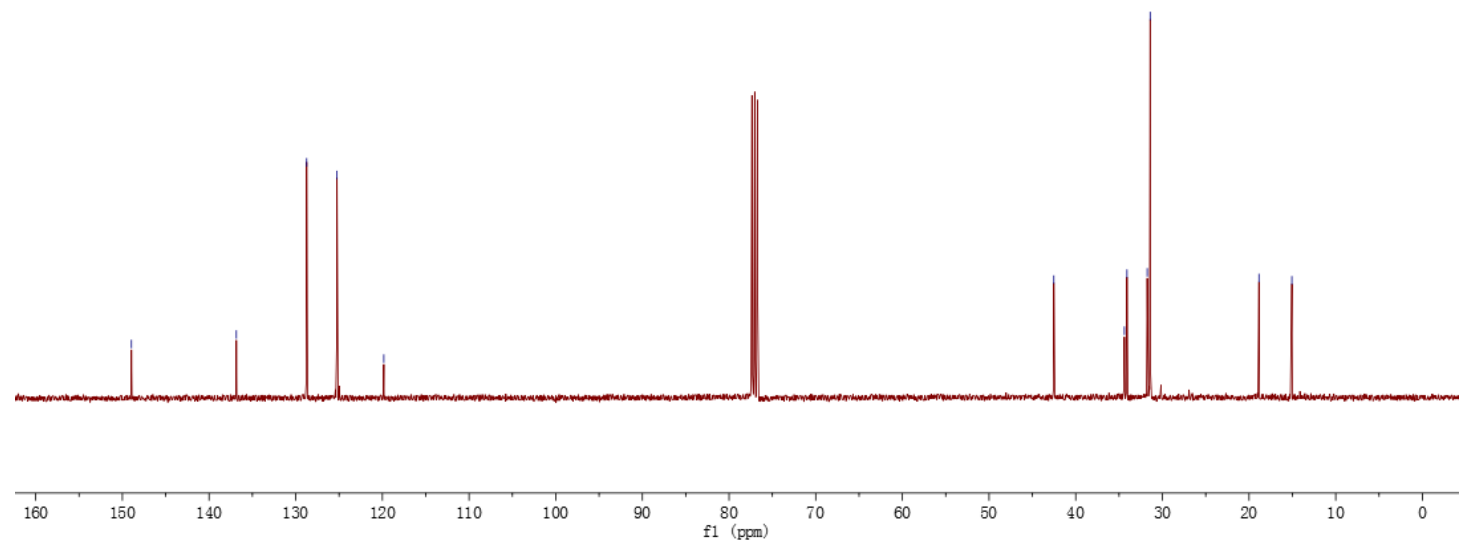
${ }^{1} \mathrm{H}$ NMR $\left(400 \mathrm{MHz}, \mathrm{CDCl}_{3}\right)$ of $\mathbf{5} \mathbf{c}$

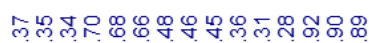

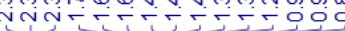

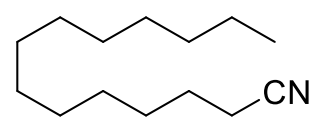
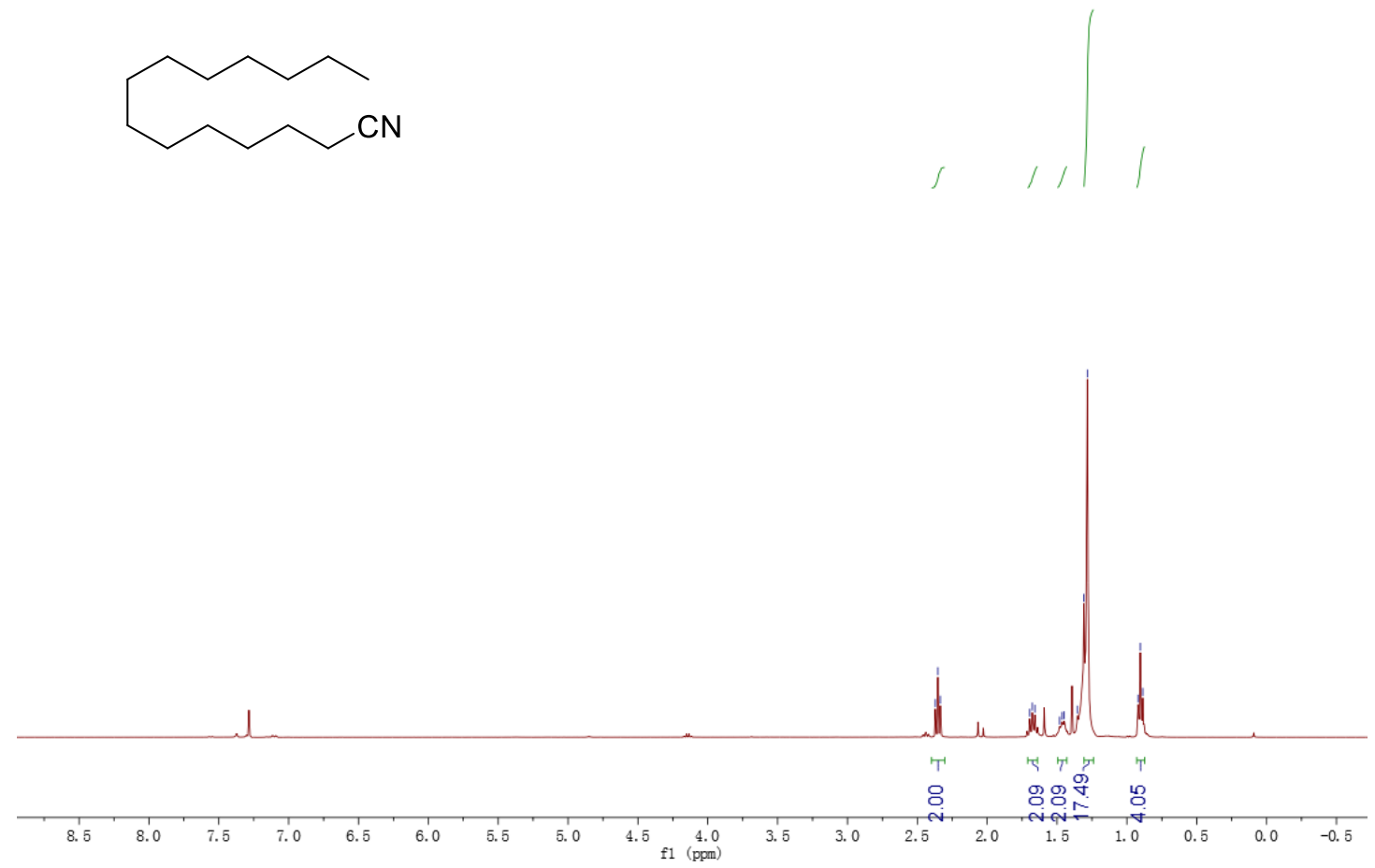

${ }^{13} \mathrm{C}\{1 \mathrm{H}\} \mathrm{NMR}\left(100 \mathrm{MHz}, \mathrm{CDCl}_{3}\right)$ of $\mathbf{5 c}$

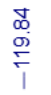

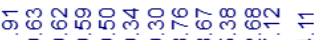
西

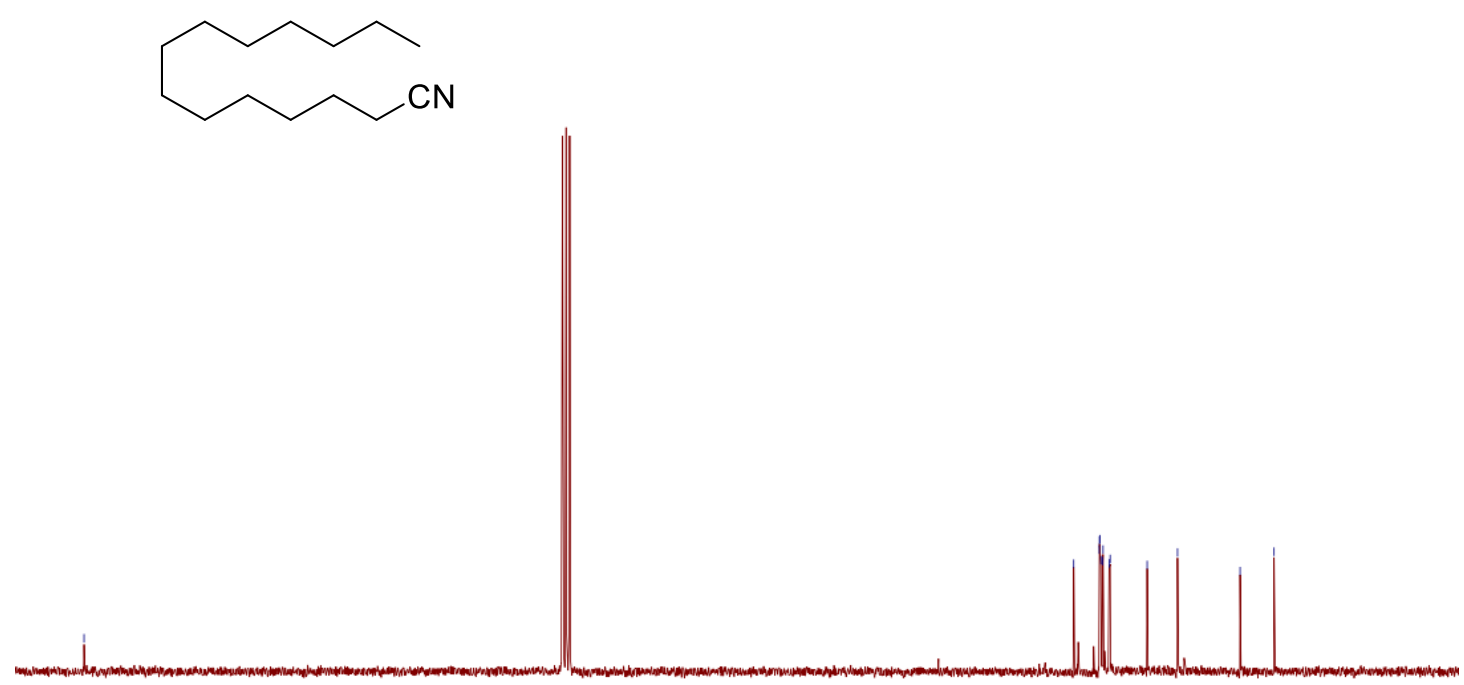

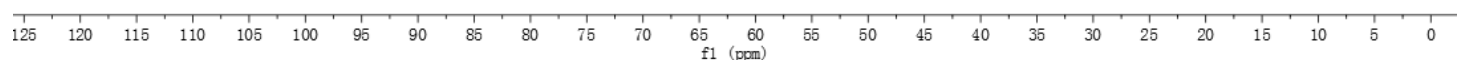


${ }^{1} \mathrm{H}$ NMR (400 MHz, $\left.\mathrm{CDCl}_{3}\right)$ of $\mathbf{5 d}$

둥요

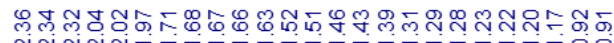

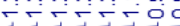<smiles>CC(C)=CCCC(C)CCCC#N</smiles>
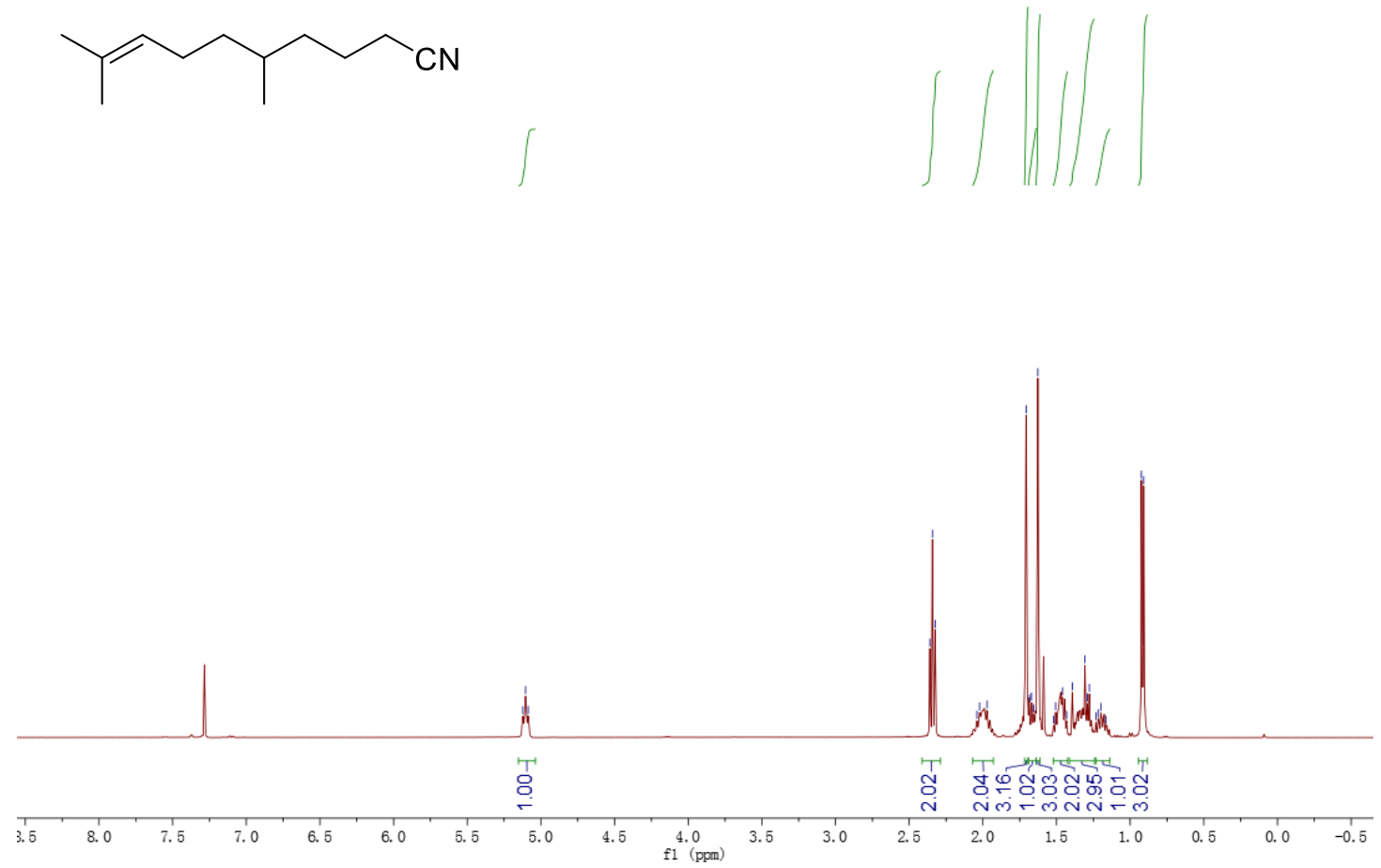

${ }^{13} \mathrm{C}\{1 \mathrm{H}\} \mathrm{NMR}\left(100 \mathrm{MHz}, \mathrm{CDCl}_{3}\right)$ of $\mathbf{5 d}$

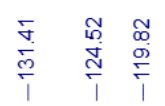

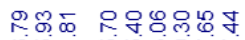

फ़ं<smiles>CC(C)=CCCC(C)CCCC#N</smiles>

$$
\|
$$

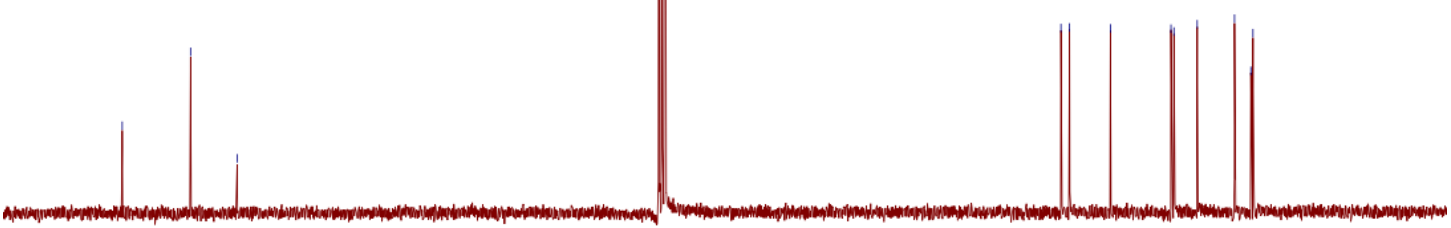

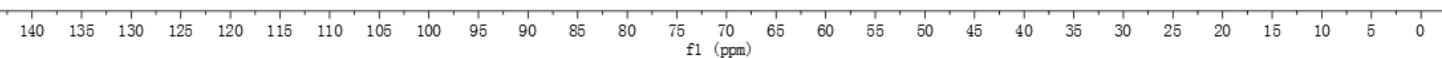


${ }^{1} \mathrm{H}$ NMR $\left(400 \mathrm{MHz}, \mathrm{CDCl}_{3}\right)$ of $5 \mathbf{e}$

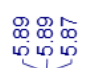

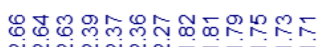

nnninnn
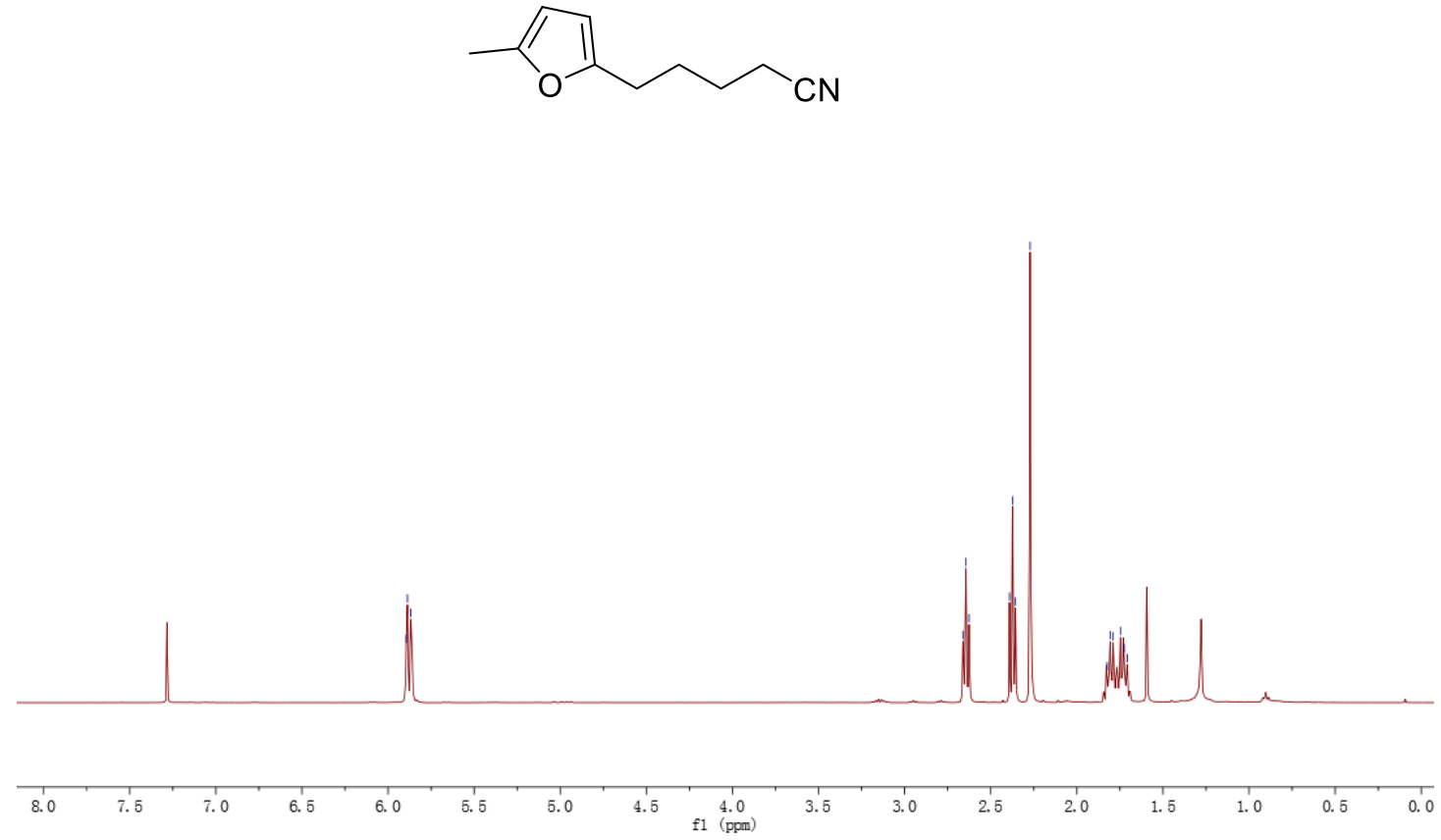

${ }^{13} \mathrm{C}\{1 \mathrm{H}\} \mathrm{NMR}\left(100 \mathrm{MHz}, \mathrm{CDCl}_{3}\right)$ of $\mathbf{5 e}$
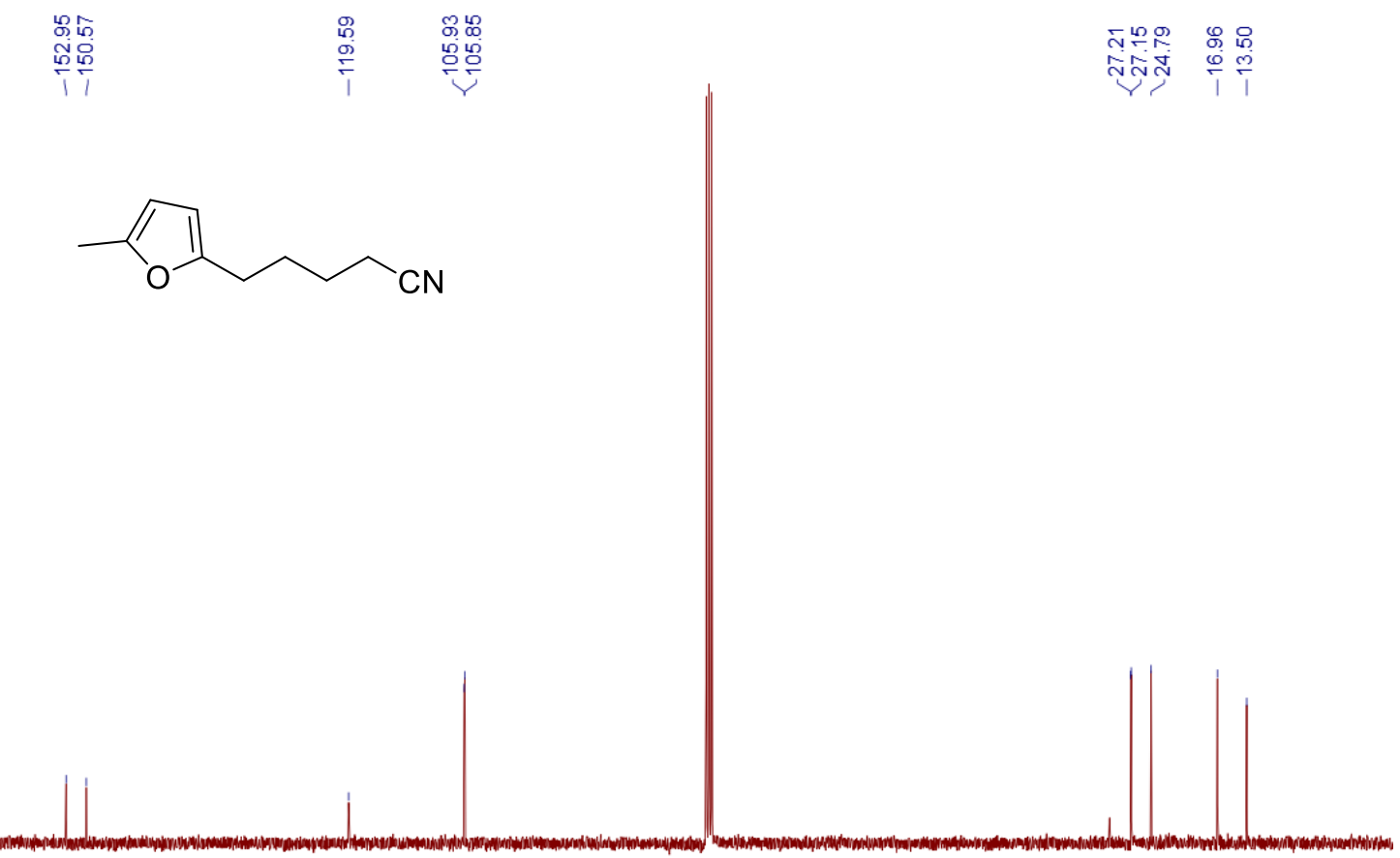

$\stackrel{7}{160}$

$5 \%$
0
0

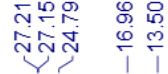
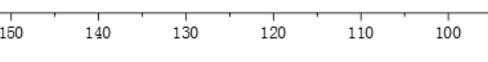

80
$\mathrm{f} 1(\mathrm{ppm})$ 
${ }^{1} \mathrm{H}$ NMR $\left(400 \mathrm{MHz}, \mathrm{CDCl}_{3}\right)$ of $\mathbf{5 f}$

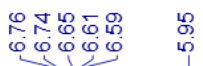

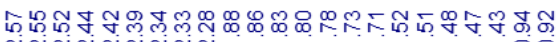

$\rightarrow 1 /$

innกinin

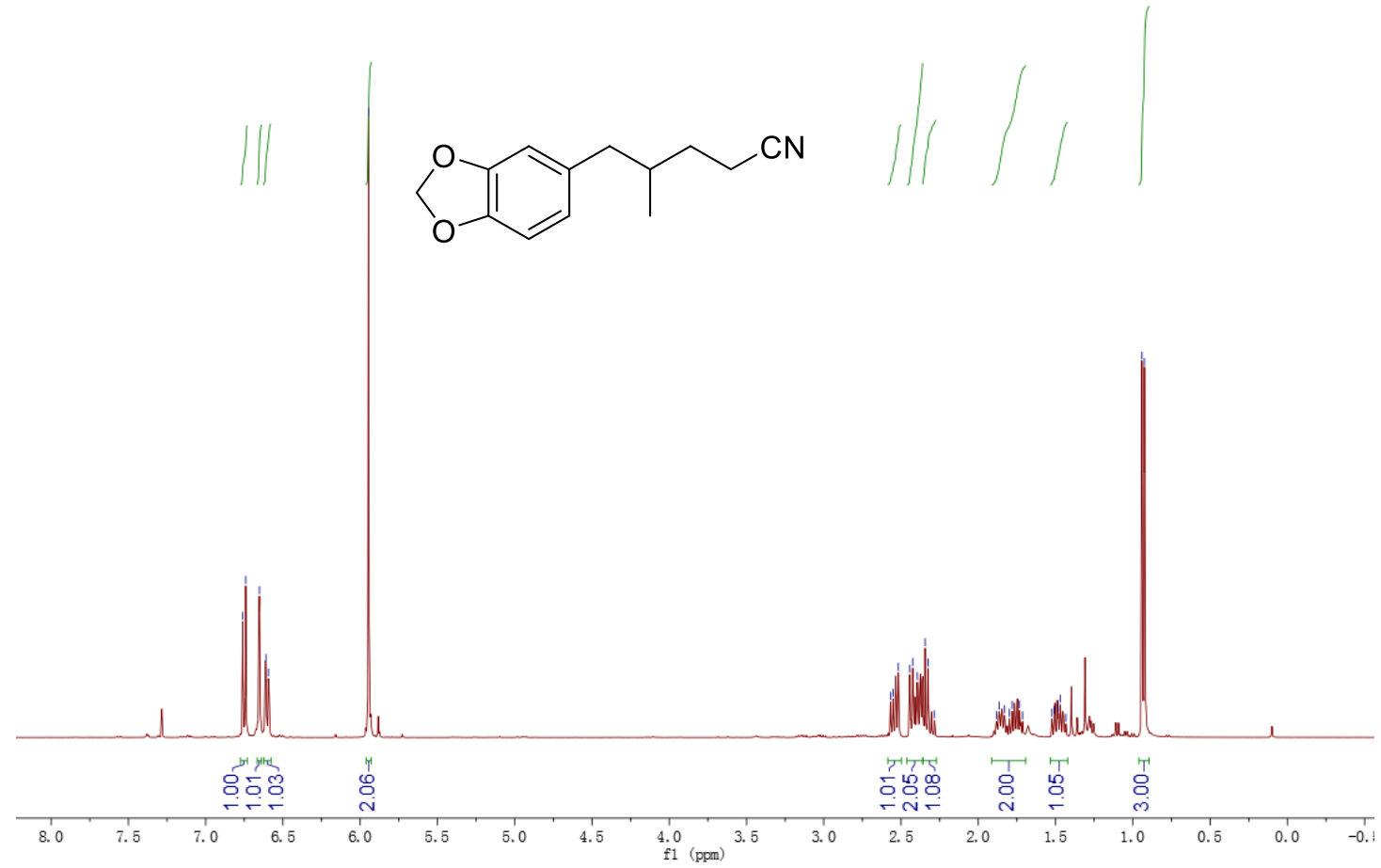

${ }^{13} \mathrm{C}\{1 \mathrm{H}\}$ NMR $\left(100 \mathrm{MHz}, \mathrm{CDCl}_{3}\right)$ of $\mathbf{5 f}$

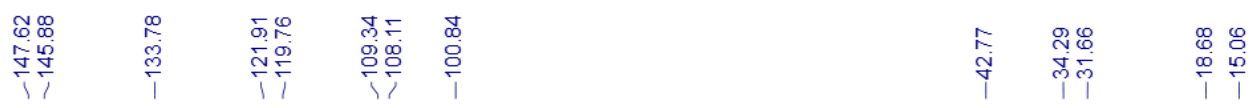<smiles>CC(CCC#N)Cc1ccc2c(c1)OCO2</smiles>

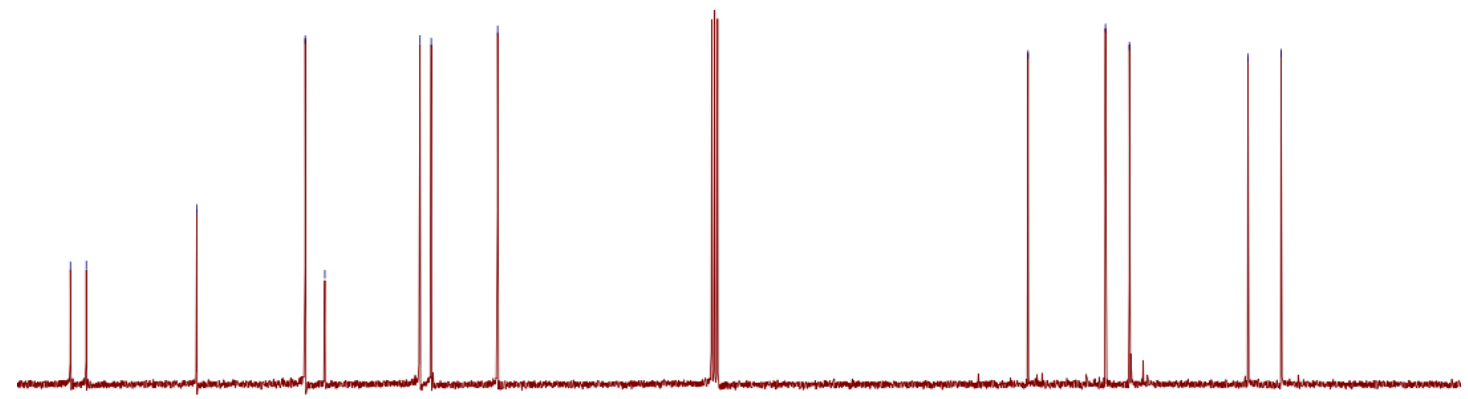

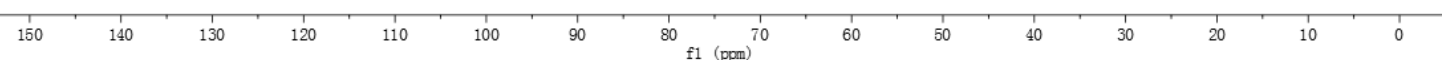


${ }^{1} \mathrm{H}$ NMR (400 MHz, $\left.\mathrm{CDCl}_{3}\right)$ of $\mathbf{5 g}$

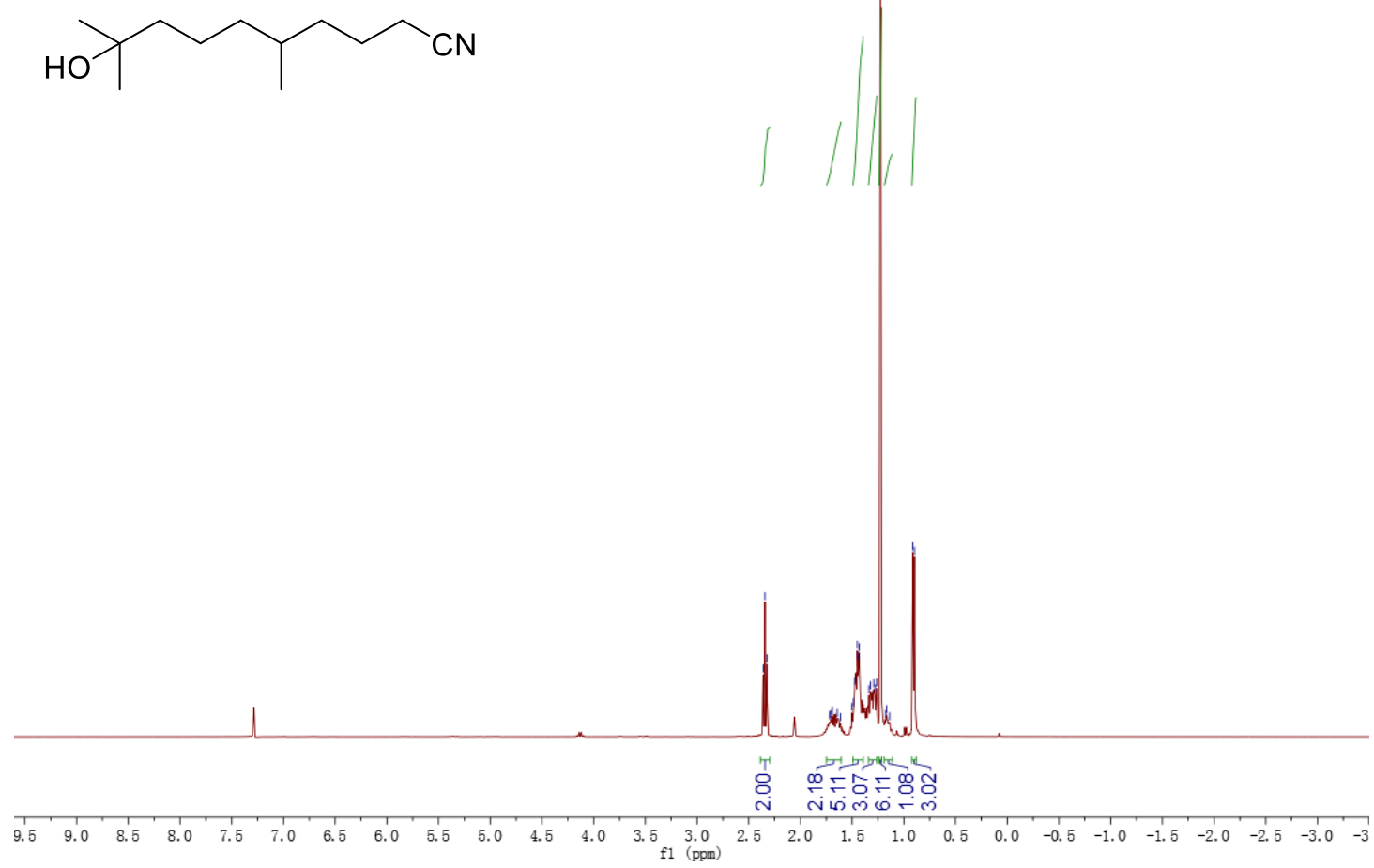

${ }^{13} \mathrm{C}\{1 \mathrm{H}\}$ NMR $\left(100 \mathrm{MHz}, \mathrm{CDCl}_{3}\right)$ of $\mathbf{5 g}$

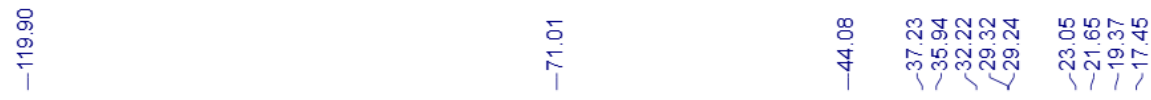<smiles>CC(CCCC#N)CCCC(C)(C)O</smiles>

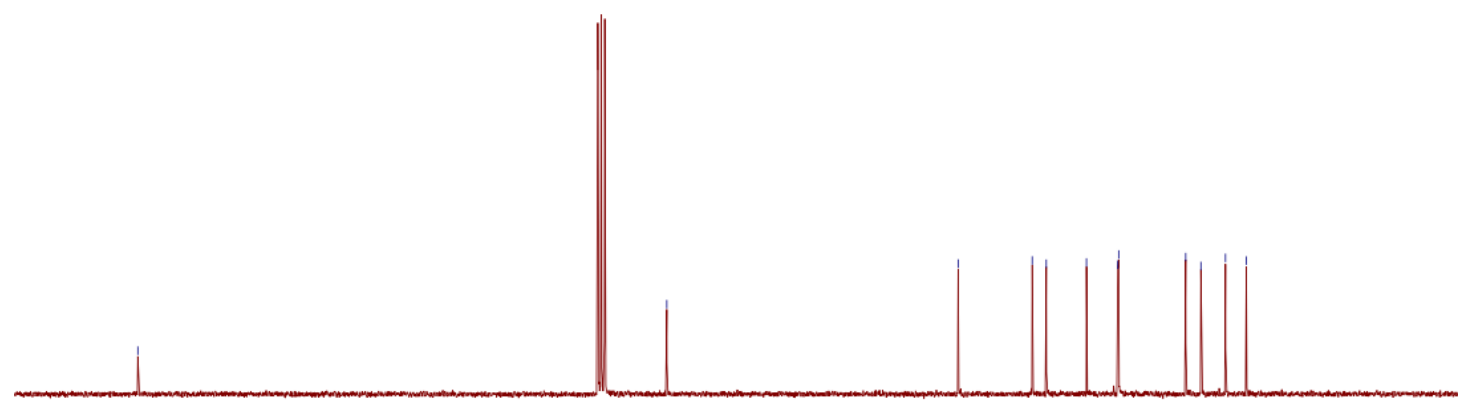

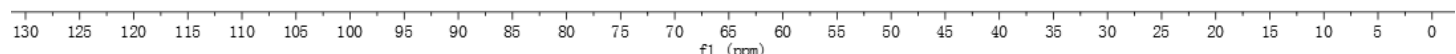


${ }^{1} \mathrm{H}$ NMR (400 MHz, $\left.\mathrm{CDCl}_{3}\right)$ of $\mathbf{5 h}$<smiles>CCOC(=O)CCCN(C)C(=O)OCc1ccccc1</smiles>

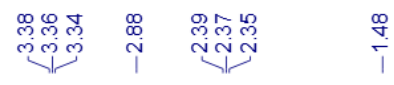

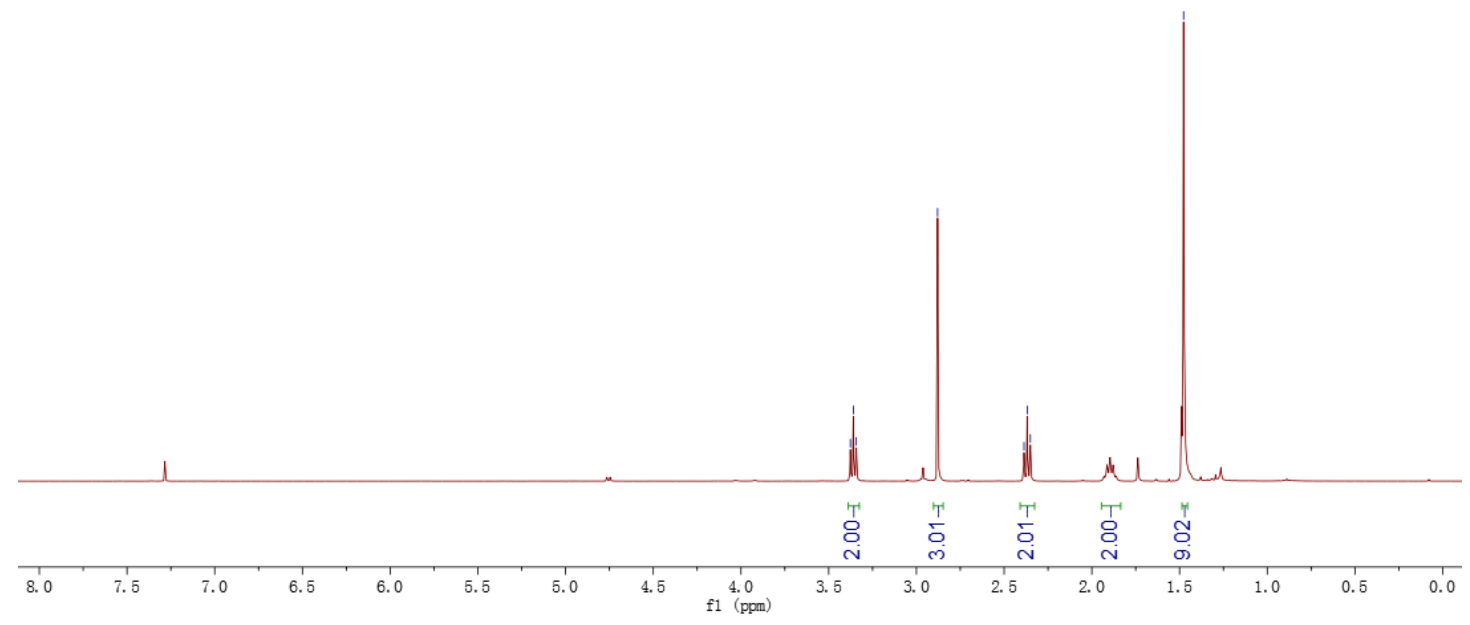

${ }^{13} \mathrm{C}\{1 \mathrm{H}\}$ NMR $\left(100 \mathrm{MHz}, \mathrm{CDCl}_{3}\right)$ of $\mathbf{5 h}$
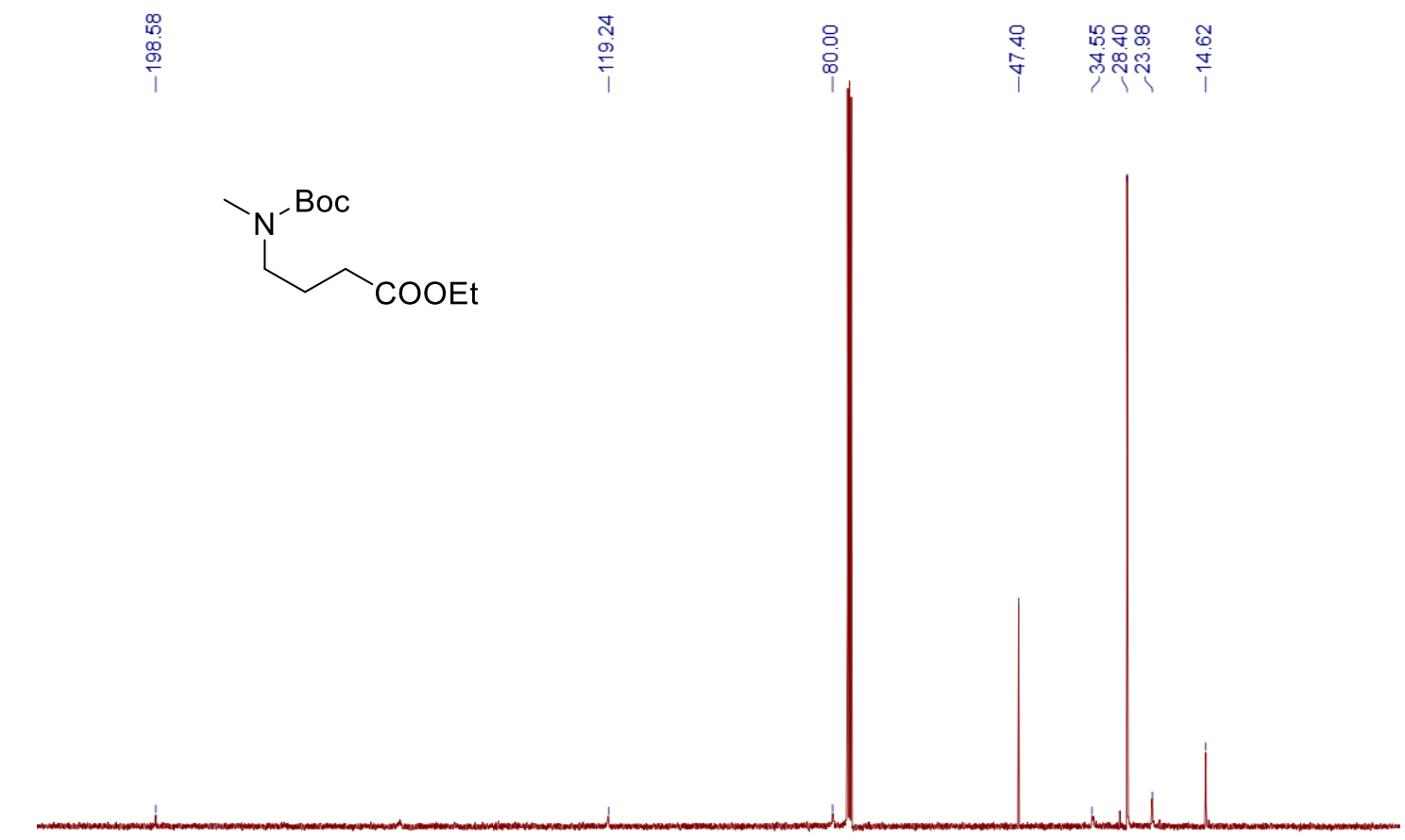

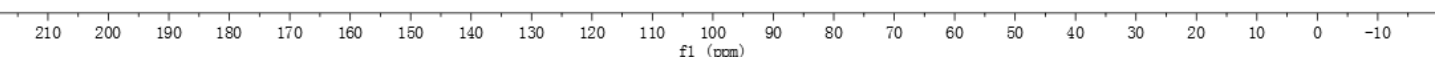


${ }^{1} \mathrm{H}$ NMR $\left(400 \mathrm{MHz}, \mathrm{CDCl}_{3}\right)$ of $\mathbf{5 i}$

중ํㅀㅀ

मी,

7ิ-ฮำ

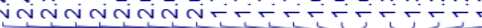<smiles>N#CCCC1CC=CCC1</smiles>
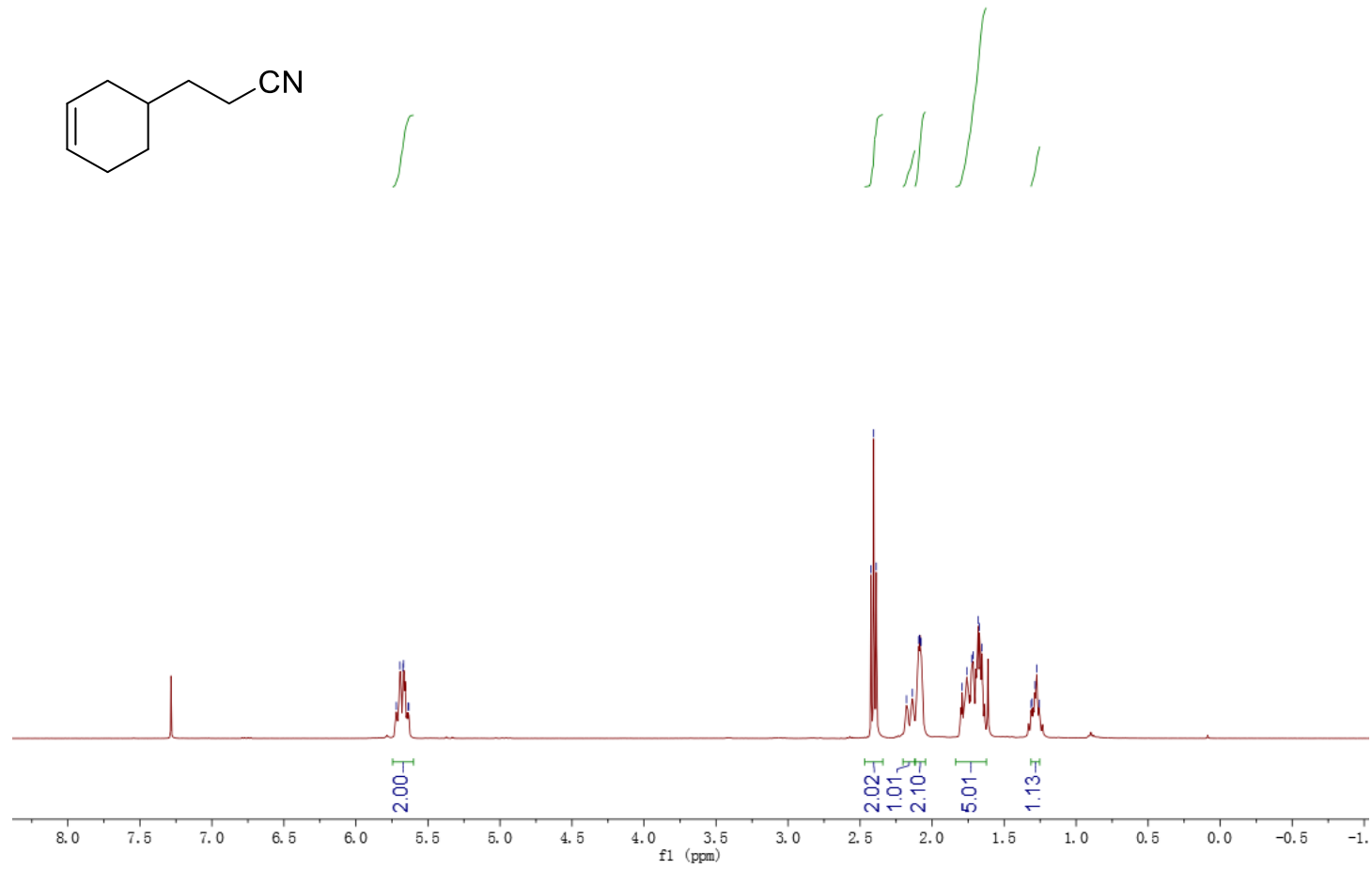

${ }^{13} \mathrm{C}\{1 \mathrm{H}\}$ NMR $\left(100 \mathrm{MHz}, \mathrm{CDCl}_{3}\right)$ of $\mathbf{5 i}$

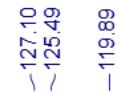

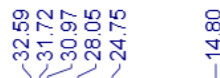<smiles>N#CCCC1CC=CCC1</smiles>

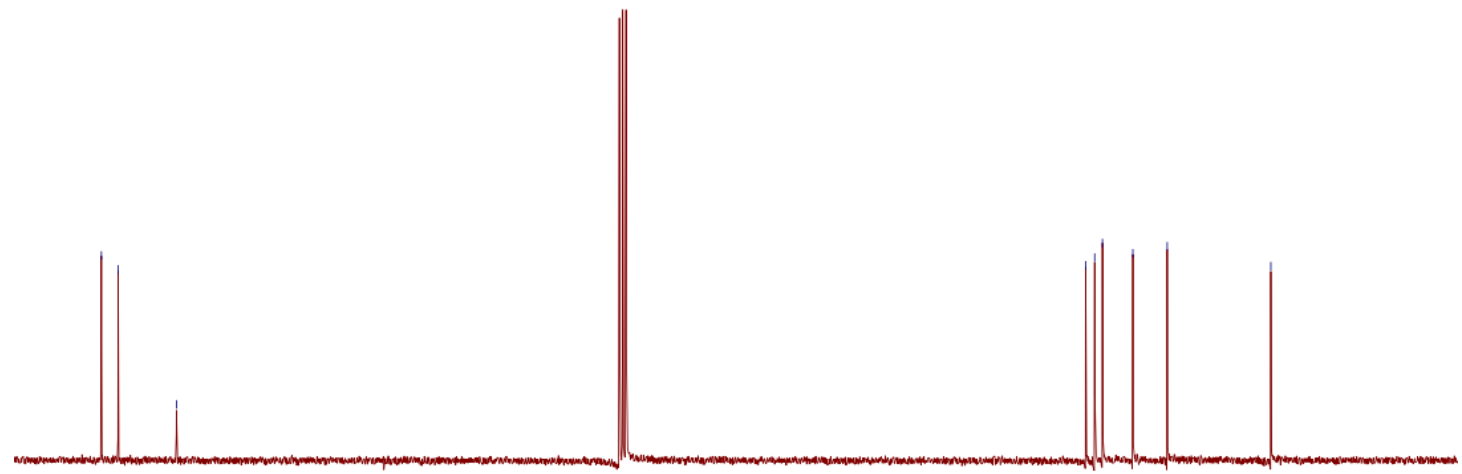

\begin{tabular}{llllllllllllllllllllllllllllllllllllllllllll}
\hline 35 & 130 & 125 & 120 & 115 & 110 & 105 & 100 & 95 & 90 & 85 & 80 & 75 & 70 & 65 & 60 & 55 & 50 & 45 & 40 & 35 & 30 & 25 & 20 & 15 & 10 & 5 & 0
\end{tabular} 
${ }^{1} \mathrm{H}$ NMR (400 MHz, $\left.\mathrm{CDCl}_{3}\right)$ of $\mathbf{5 j}$
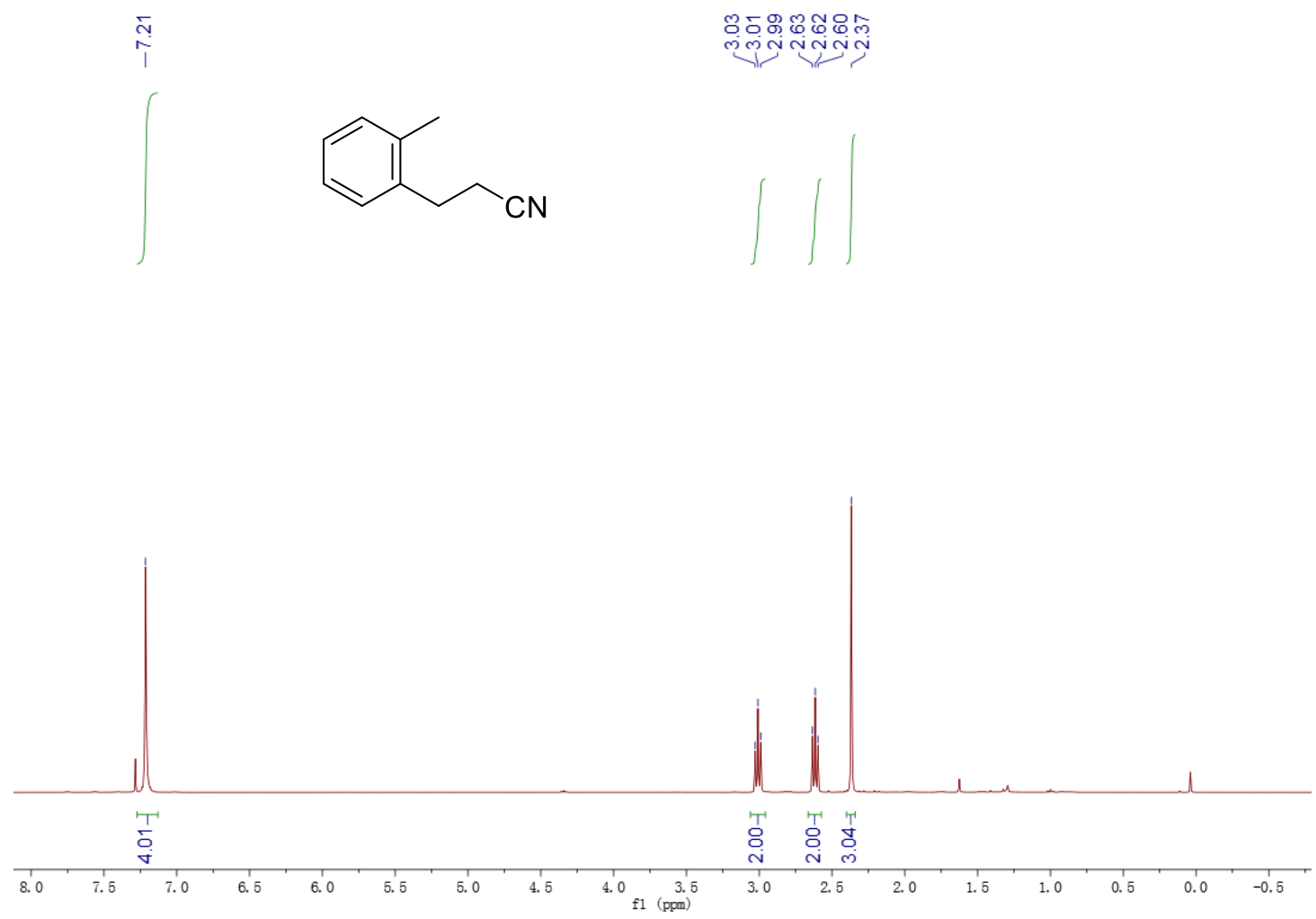

${ }^{13} \mathrm{C}\{1 \mathrm{H}\}$ NMR $\left(100 \mathrm{MHz}, \mathrm{CDCl}_{3}\right)$ of $\mathbf{5 j}$

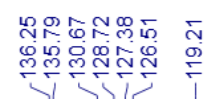

5
$\stackrel{0}{\infty}$

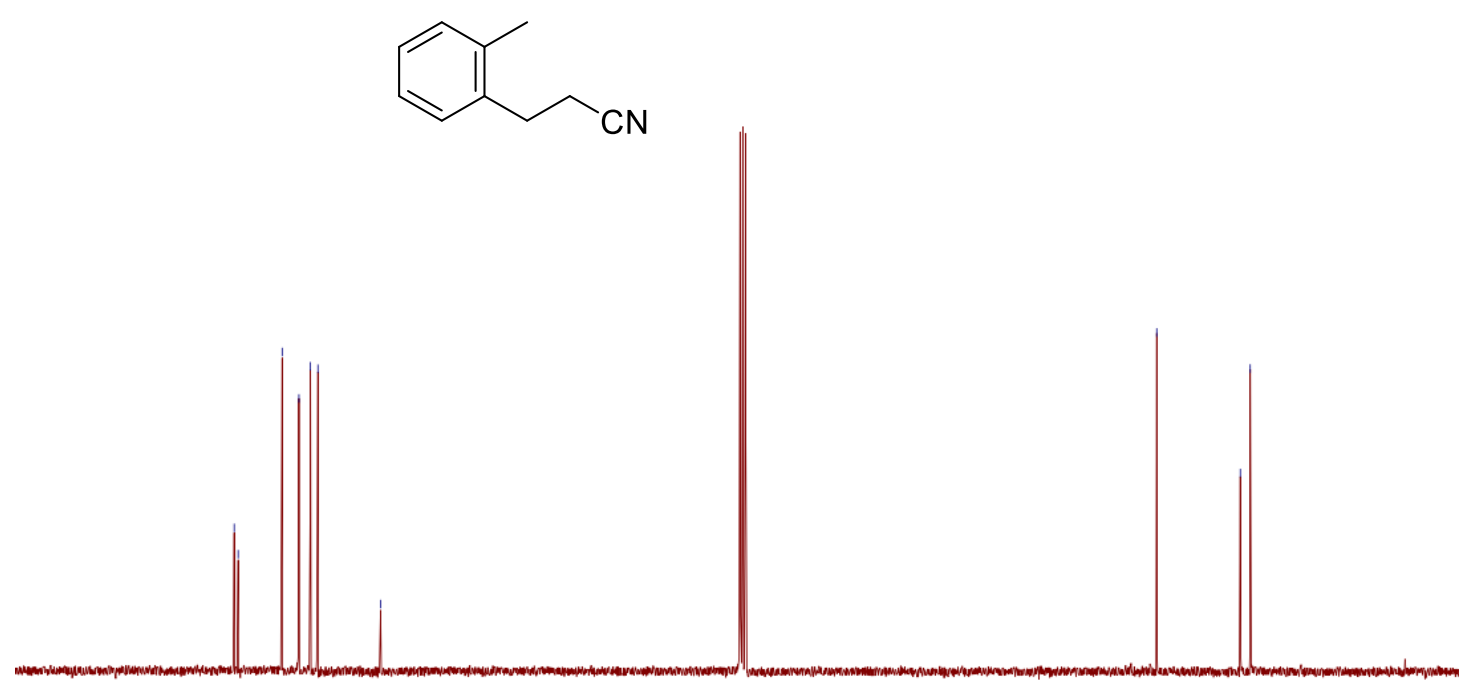

$\frac{1}{160}$
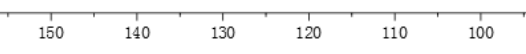

${ }_{\mathrm{f} 1(\mathrm{ppm})}^{1} 7$ 
${ }^{1} \mathrm{H}$ NMR (400 MHz, $\left.\mathrm{CDCl}_{3}\right)$ of $\mathbf{5 k}$

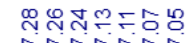

vinisin

ติ

งั่งกัง

11<smiles>Cc1cccc(CCC#N)c1</smiles>
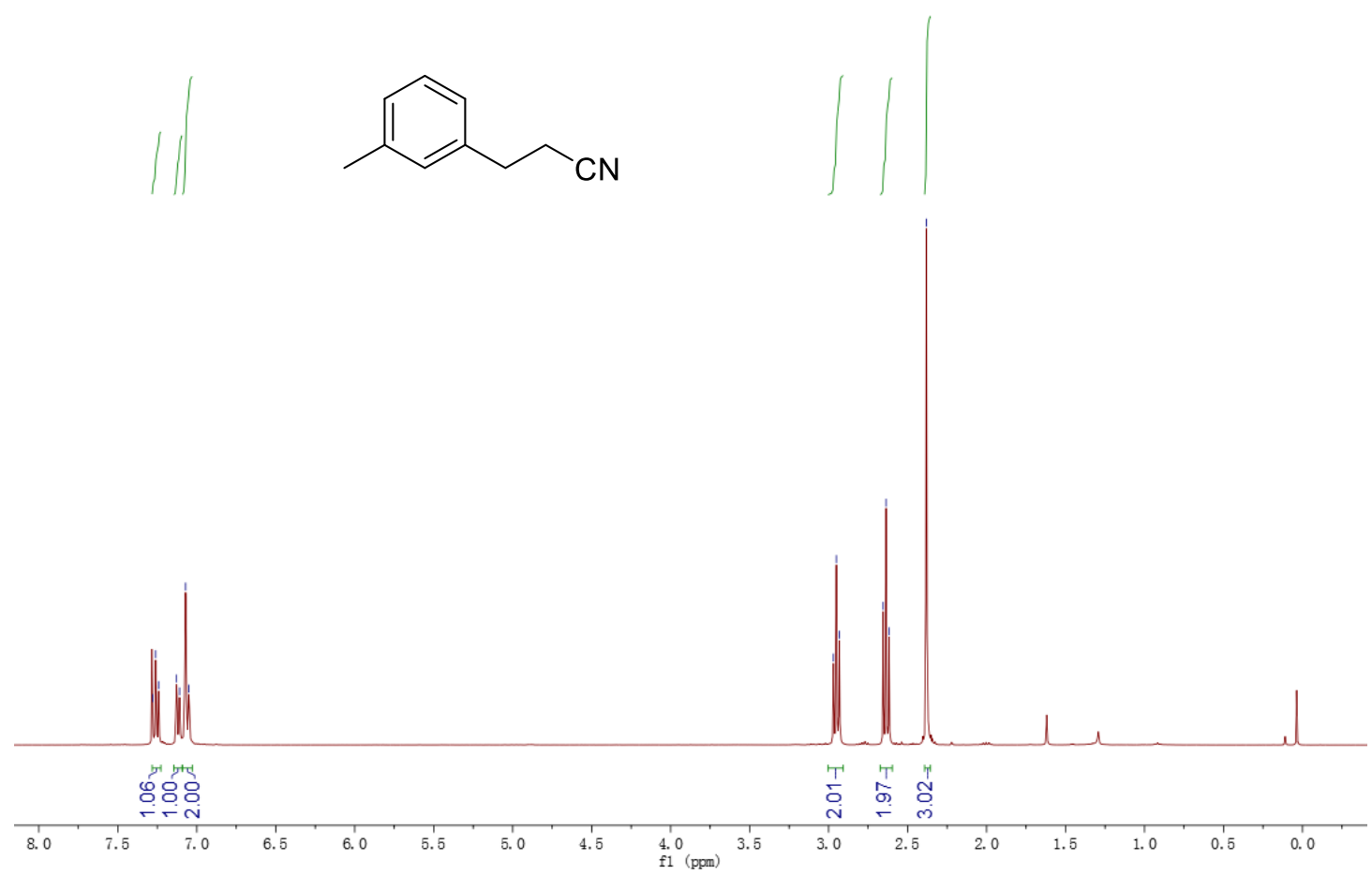

${ }^{13} \mathrm{C}\{1 \mathrm{H}\}$ NMR $\left(100 \mathrm{MHz}, \mathrm{CDCl}_{3}\right)$ of $\mathbf{5} \mathbf{k}$

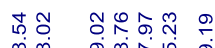

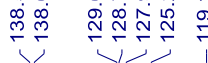

菖
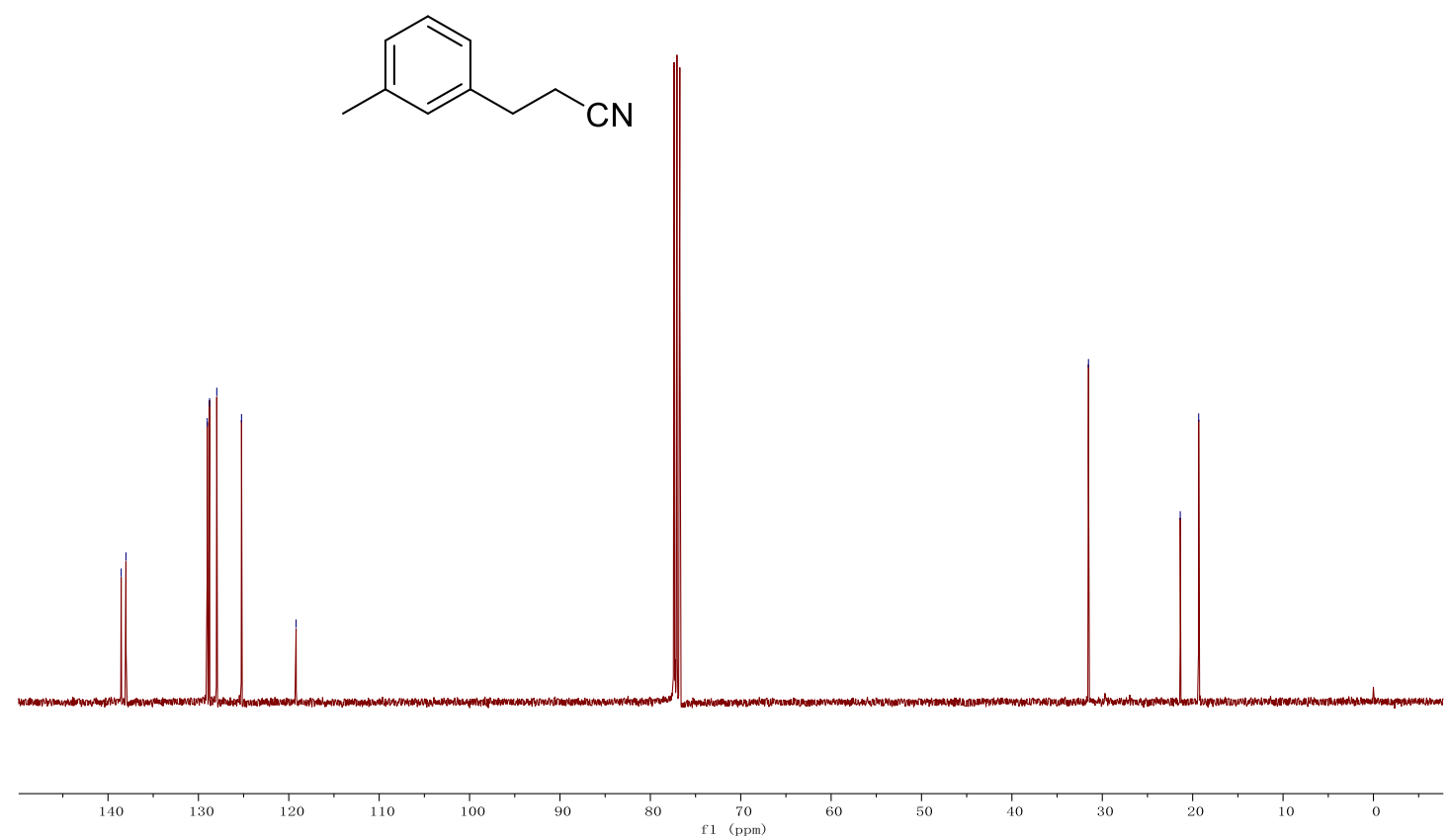
${ }^{1} \mathrm{H}$ NMR (400 MHz, $\left.\mathrm{CDCl}_{3}\right)$ of $\mathbf{5 l}$

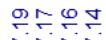

inis

คิ่

$\sqrt[n]{44 n \sin 4}$<smiles>Cc1ccc(CCC#N)cc1</smiles>
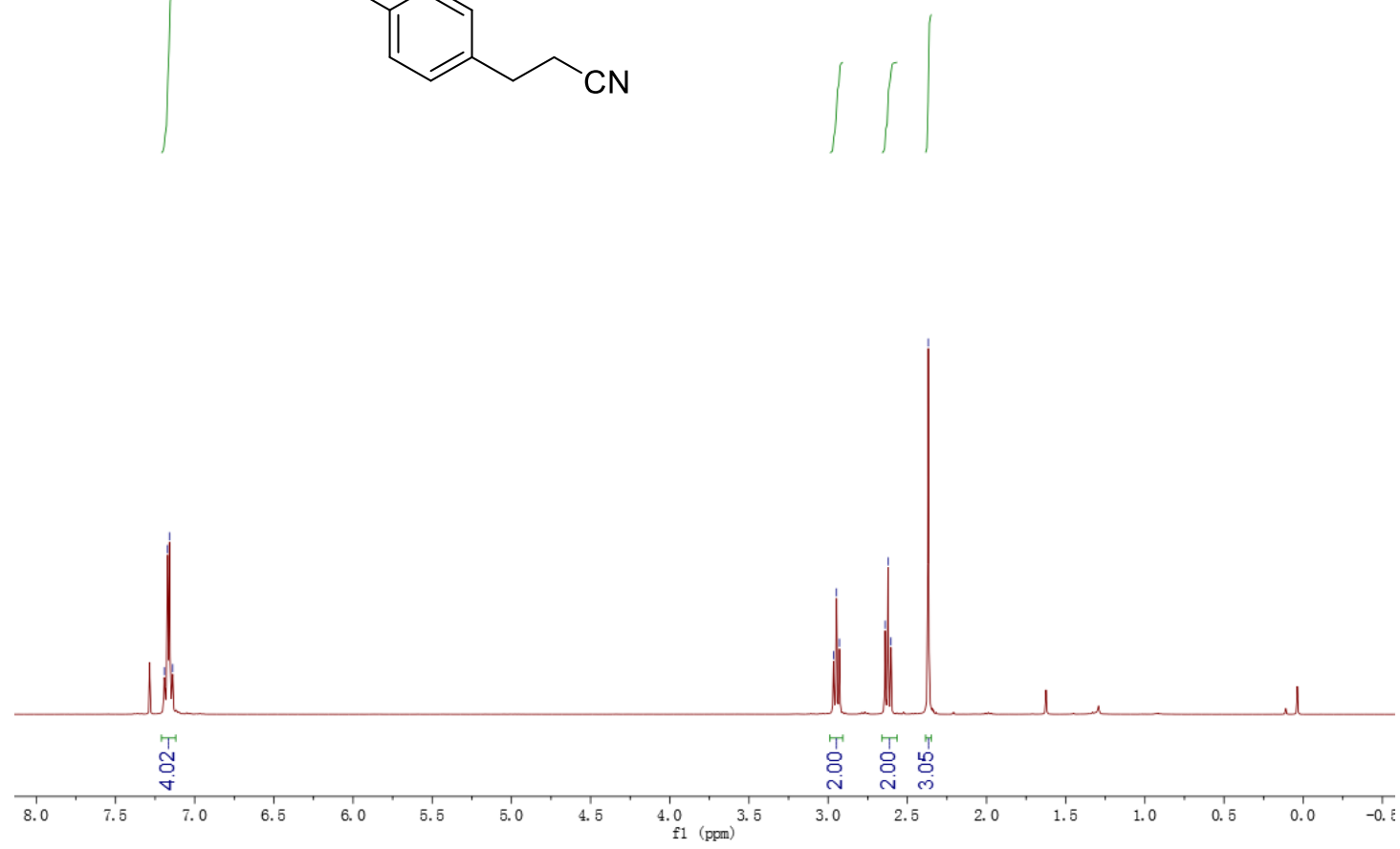

${ }^{13} \mathrm{C}\{1 \mathrm{H}\} \mathrm{NMR}\left(100 \mathrm{MHz}, \mathrm{CDCl}_{3}\right)$ of $\mathbf{5} \mathbf{I}$
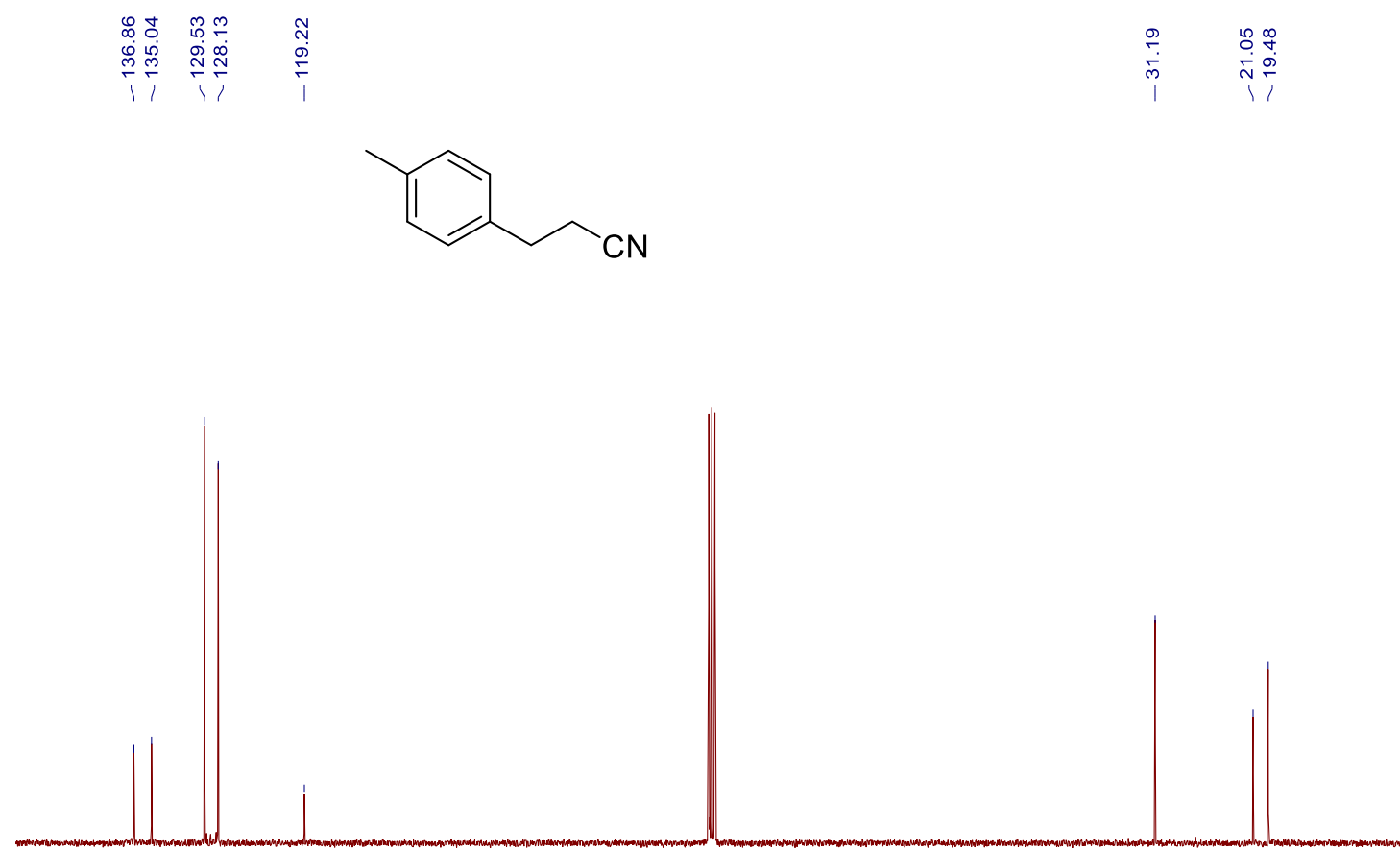

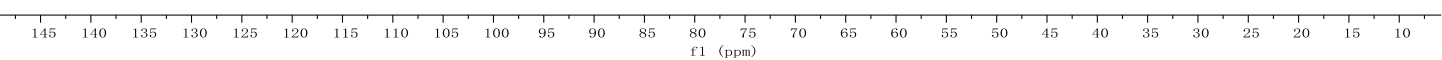


${ }^{1} \mathrm{H}$ NMR $\left(400 \mathrm{MHz}, \mathrm{CDCl}_{3}\right)$ of $\mathbf{5 m}$

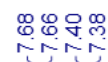

$\mathrm{NC}$<smiles>N#CCCc1ccccc1</smiles>

\section{두융요웅}

minging
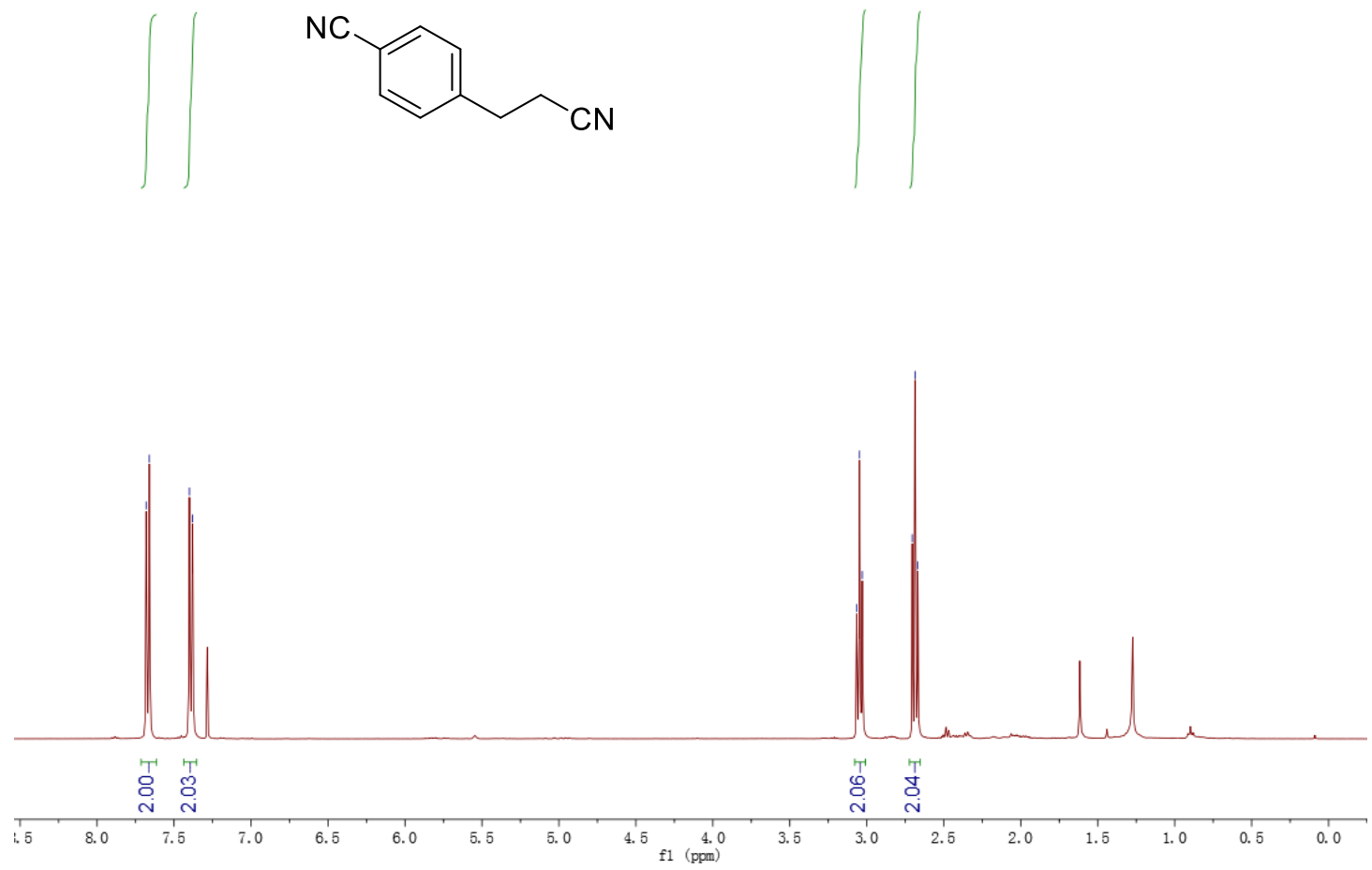

${ }^{13} \mathrm{C}\{1 \mathrm{H}\} \mathrm{NMR}\left(100 \mathrm{MHz}, \mathrm{CDCl}_{3}\right)$ of $\mathbf{5 m}$

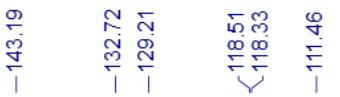

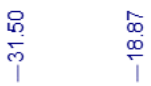<smiles>N#CCCc1ccc(C#N)cc1</smiles>

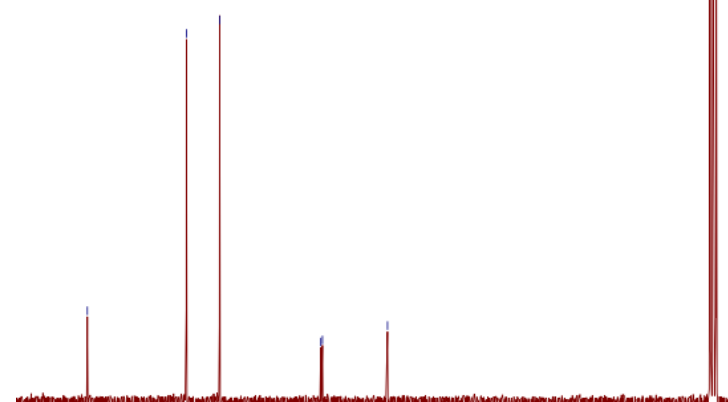

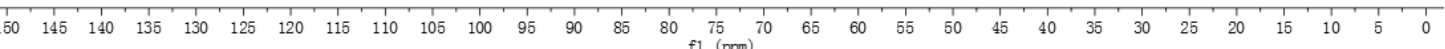


${ }^{1} \mathrm{H}$ NMR $\left(400 \mathrm{MHz}, \mathrm{CDCl}_{3}\right)$ of $\mathbf{5 n}$
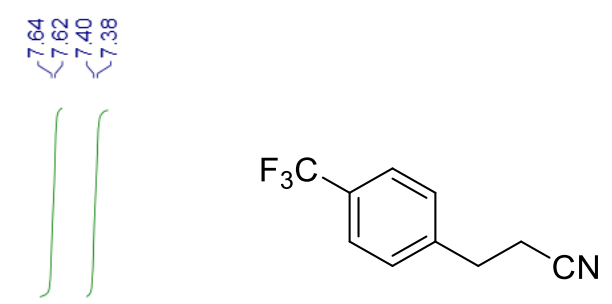

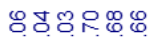

minginn
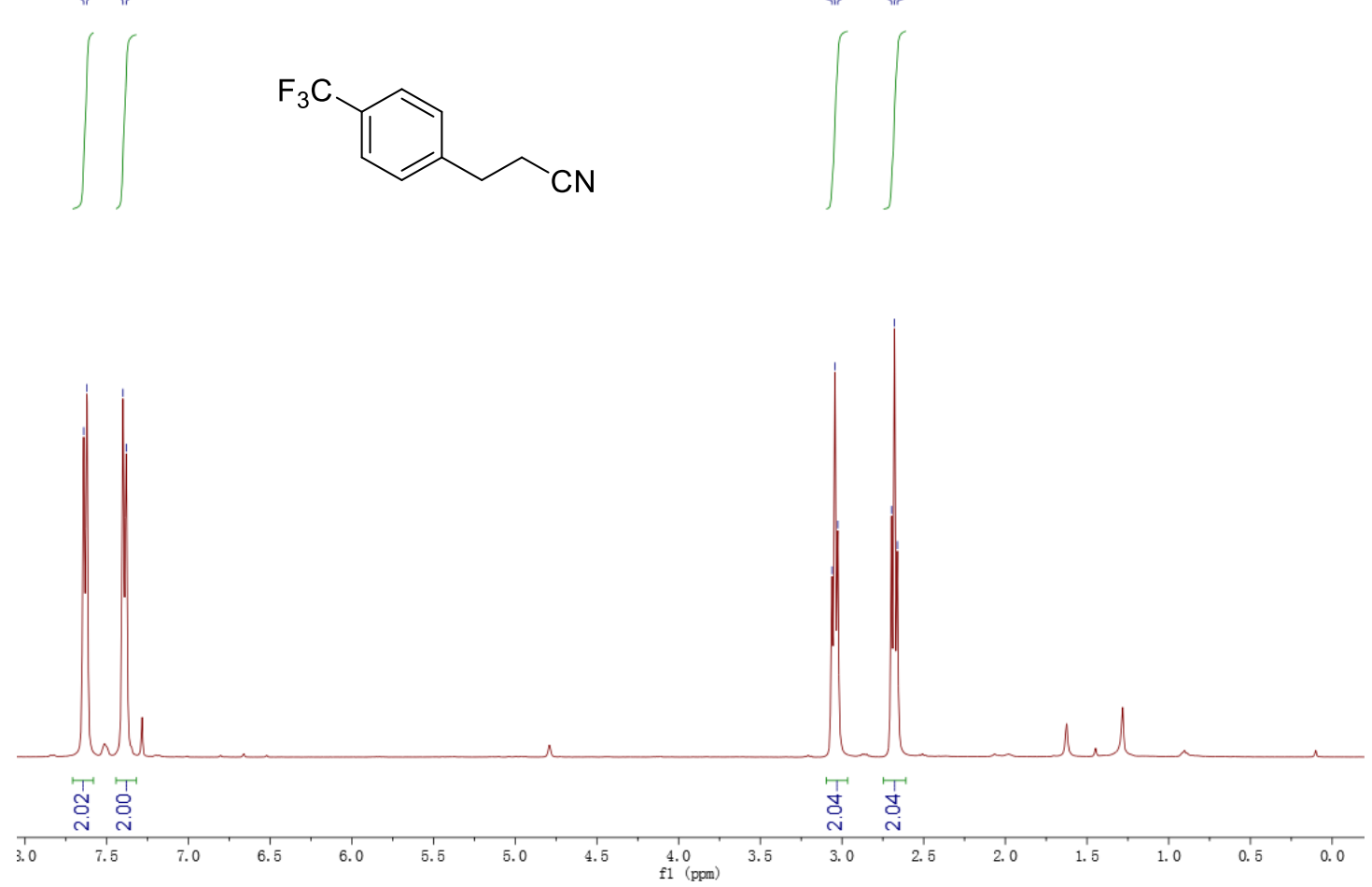

${ }^{13} \mathrm{C}\{1 \mathrm{H}\} \mathrm{NMR}\left(100 \mathrm{MHz}, \mathrm{CDCl}_{3}\right)$ of $\mathbf{5 n}$

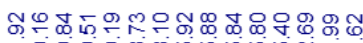

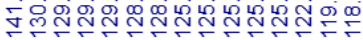<smiles>N#CCCc1ccc(C(F)(F)F)cc1</smiles>

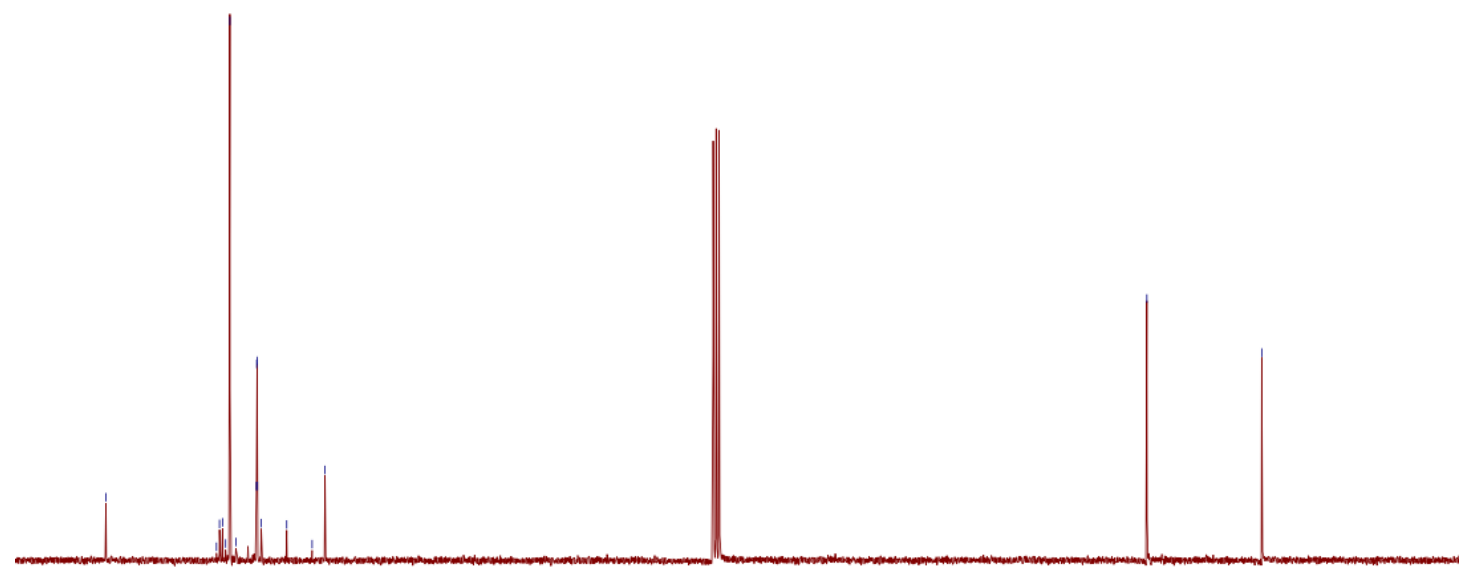

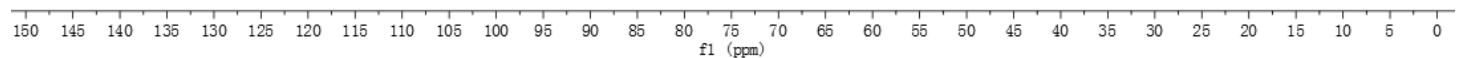


${ }^{1} \mathrm{H}$ NMR (400 MHz, $\left.\mathrm{CDCl}_{3}\right)$ of $\mathbf{5 o}$

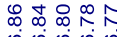

400

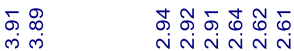

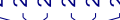

$\mathrm{MeO}$<smiles>CCc1ccc(CCC#N)cc1OC</smiles>
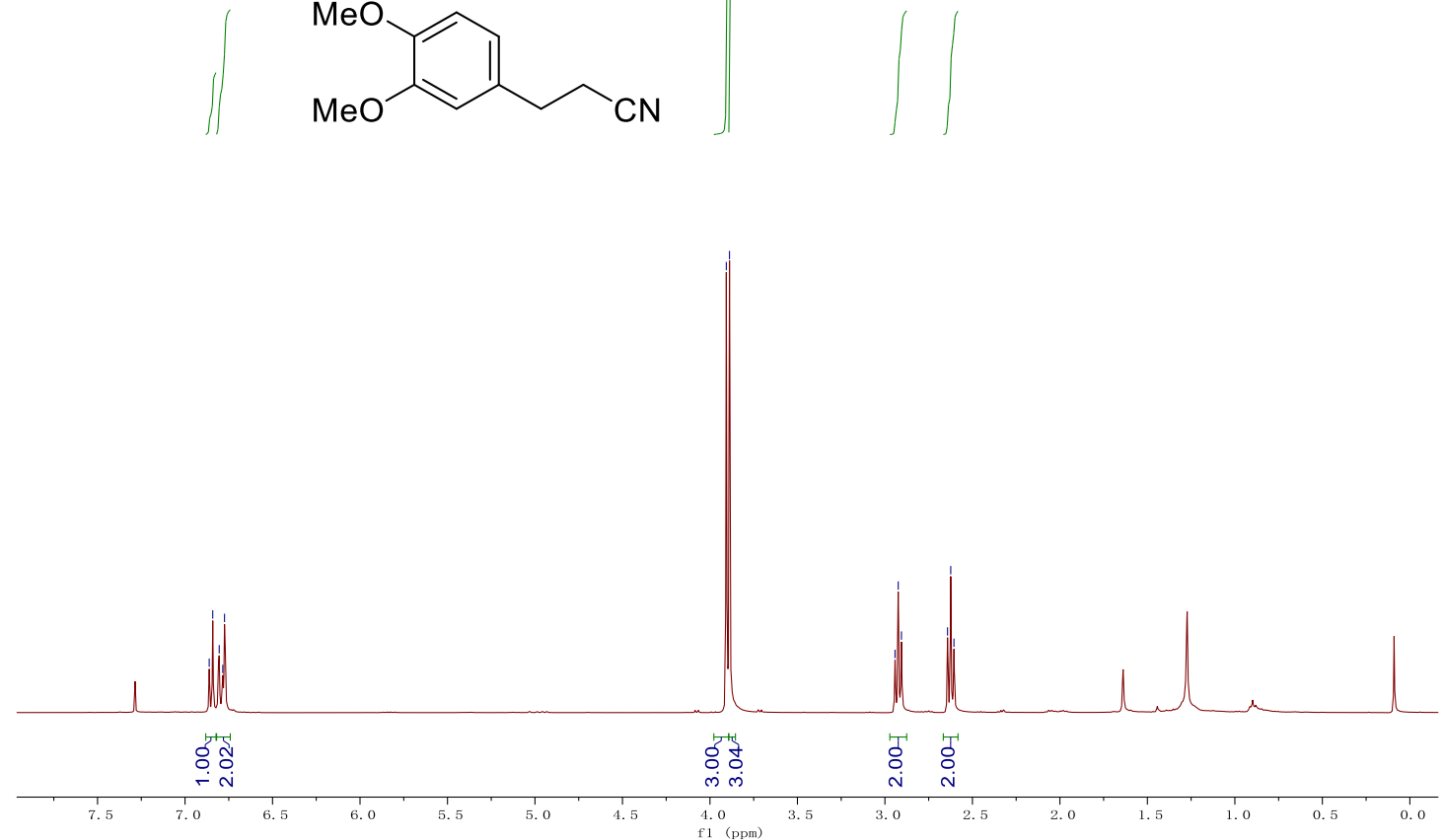

${ }^{13} \mathrm{C}\{1 \mathrm{H}\} \mathrm{NMR}\left(100 \mathrm{MHz}, \mathrm{CDCl}_{3}\right)$ of $\mathbf{5 0}$

竞望<smiles>COc1ccc(CCC#N)cc1OC</smiles>

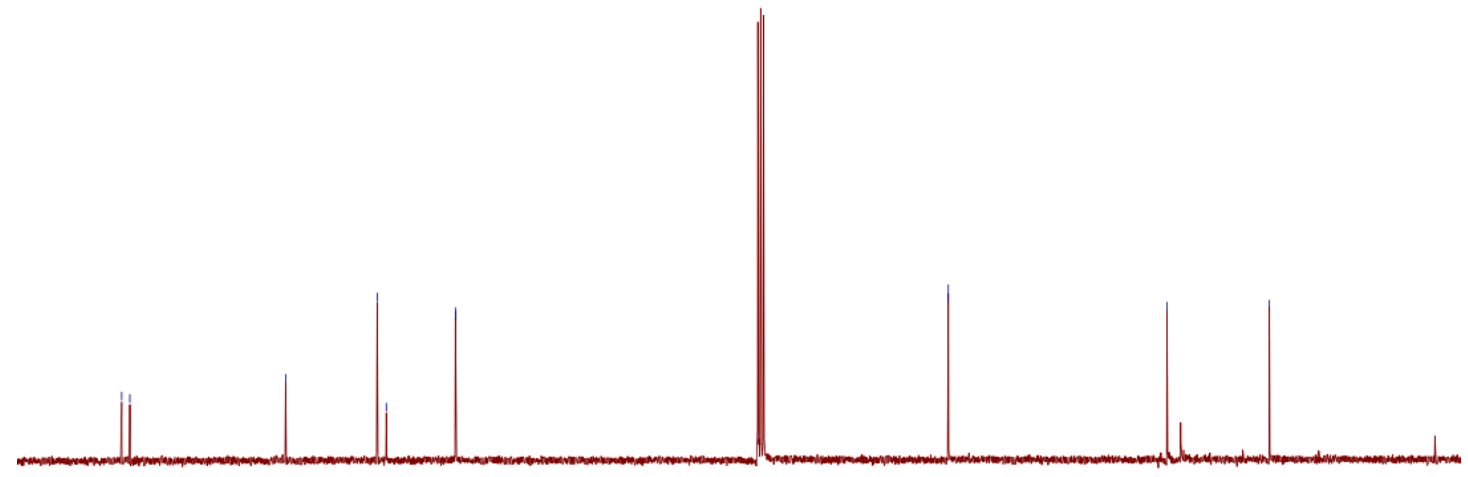

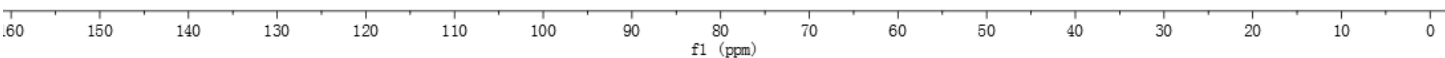


${ }^{1} \mathrm{H}$ NMR $\left(400 \mathrm{MHz}, \mathrm{CDCl}_{3}\right)$ of $\mathbf{5 p}$

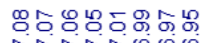

ininge

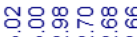

minning
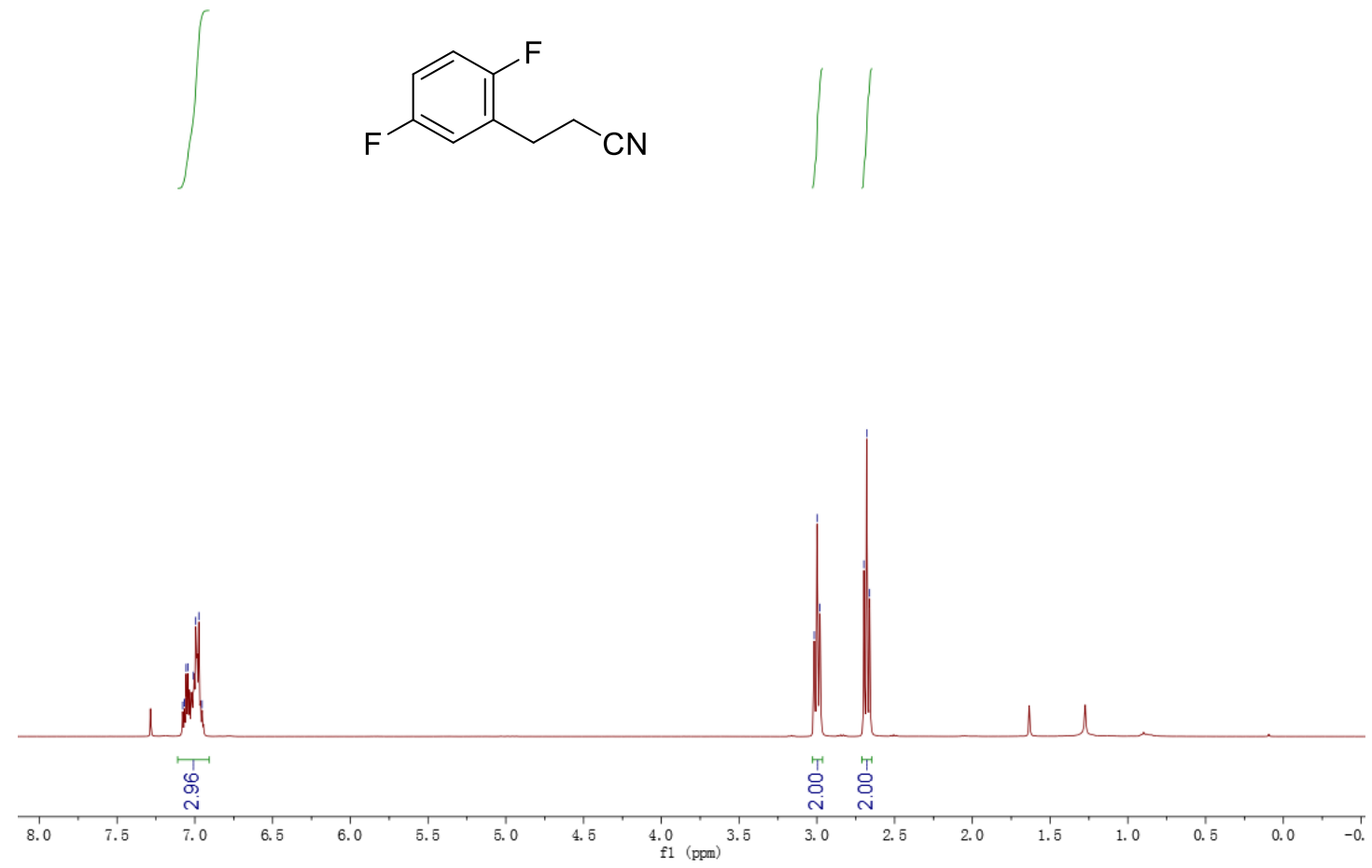

${ }^{13} \mathrm{C}\{1 \mathrm{H}\} \mathrm{NMR}\left(100 \mathrm{MHz}, \mathrm{CDCl}_{3}\right)$ of $\mathbf{5 p}$

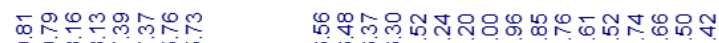

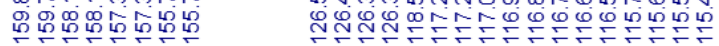

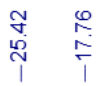

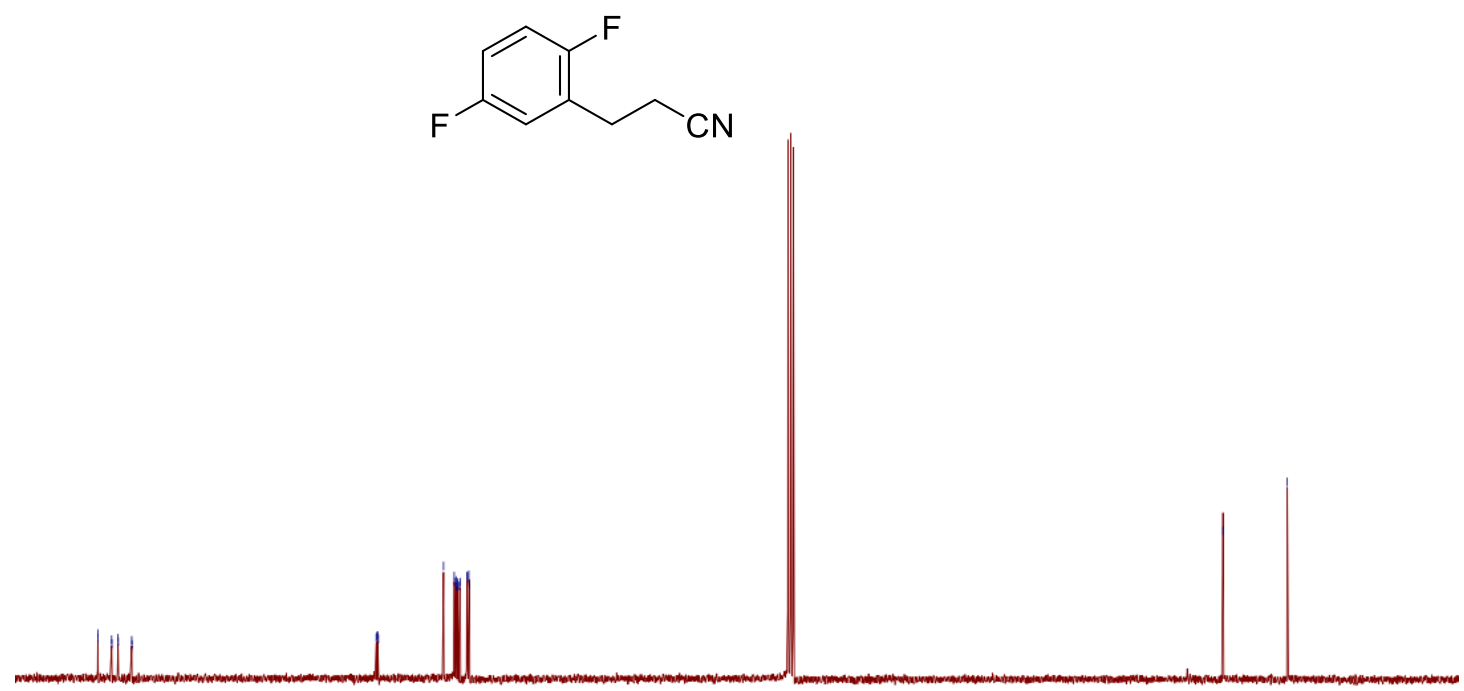

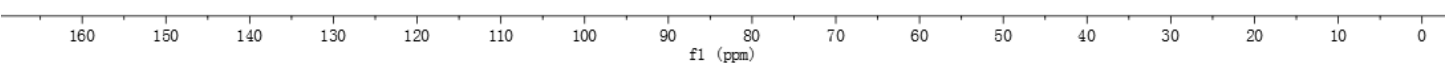


${ }^{1} \mathrm{H}$ NMR $\left(400 \mathrm{MHz}, \mathrm{CDCl}_{3}\right)$ of $\mathbf{5 q}$

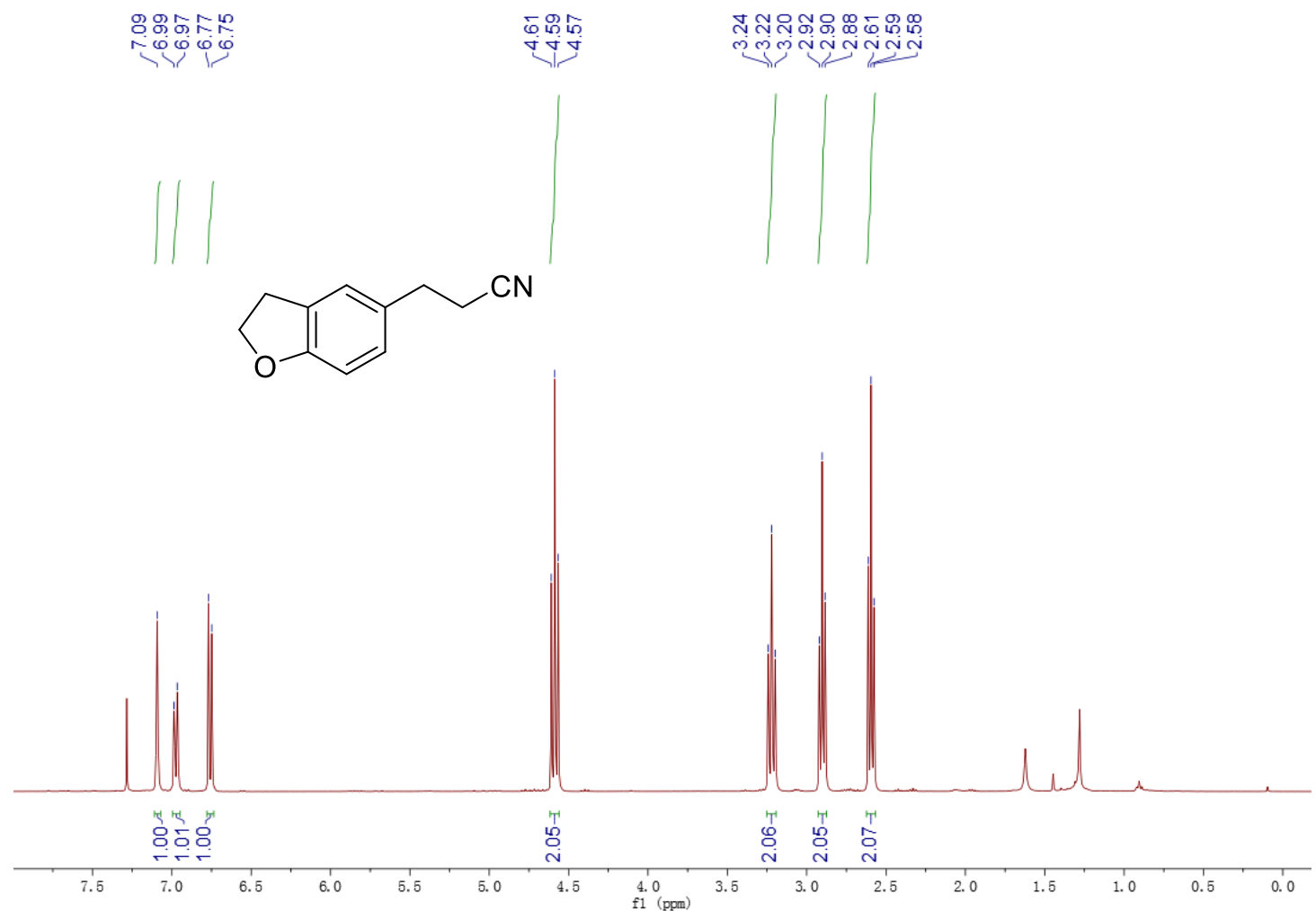

${ }^{13} \mathrm{C}\{1 \mathrm{H}\} \mathrm{NMR}\left(100 \mathrm{MHz}, \mathrm{CDCl}_{3}\right)$ of $\mathbf{5 q}$

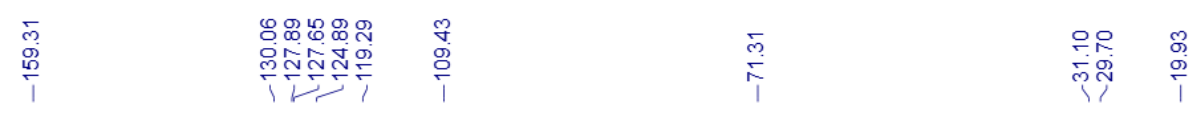<smiles>N#CCCc1ccc2c(c1)CCO2</smiles>

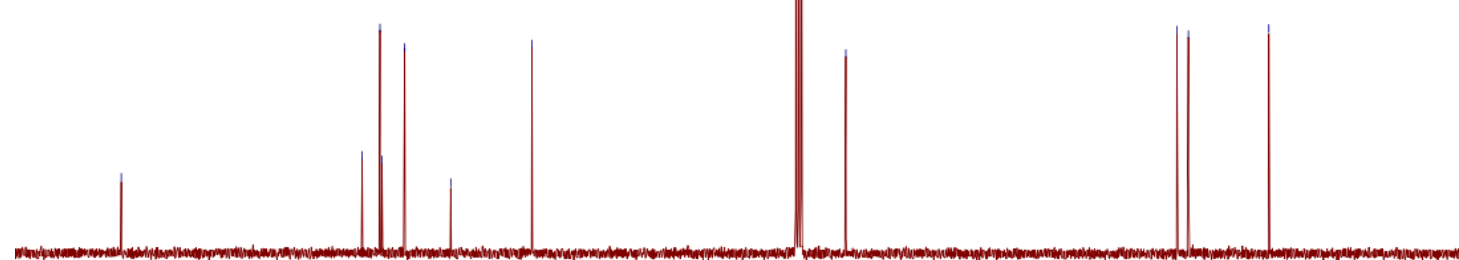

170

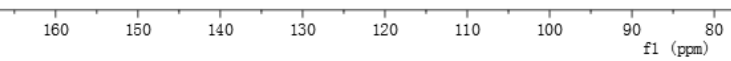


${ }^{1} \mathrm{H}$ NMR (400 MHz, $\mathrm{CDCl}_{3}$ ) of $\mathbf{5 r}$

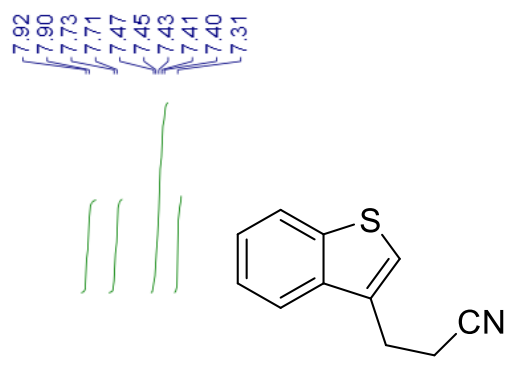

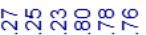

गुल Nंत

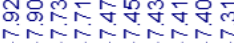
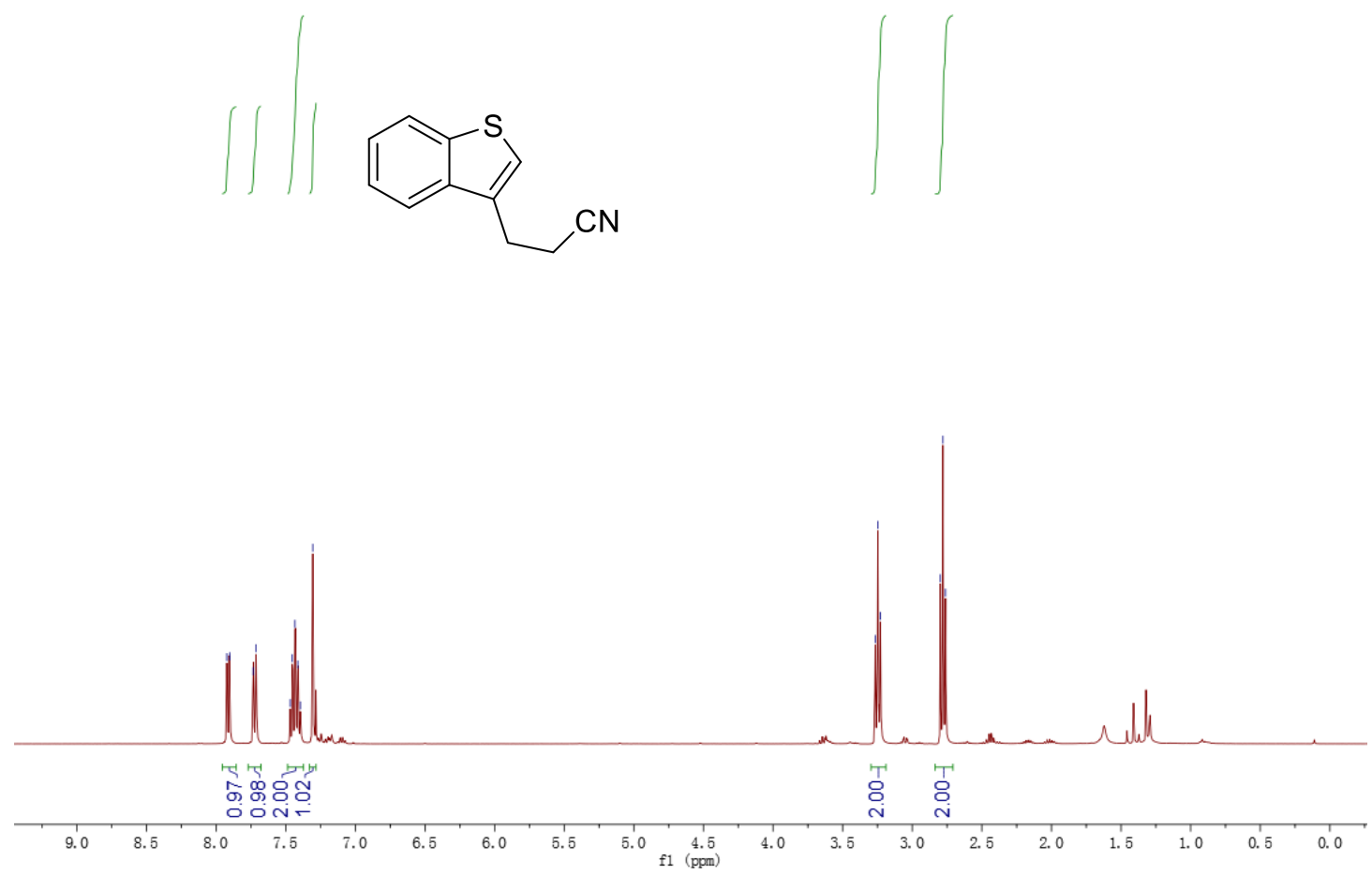

${ }^{13} \mathrm{C}\{1 \mathrm{H}\} \mathrm{NMR}\left(100 \mathrm{MHz}, \mathrm{CDCl}_{3}\right)$ of $\mathbf{5 r}$

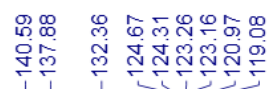

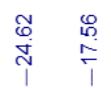<smiles>N#CCCc1csc2ccccc12</smiles>

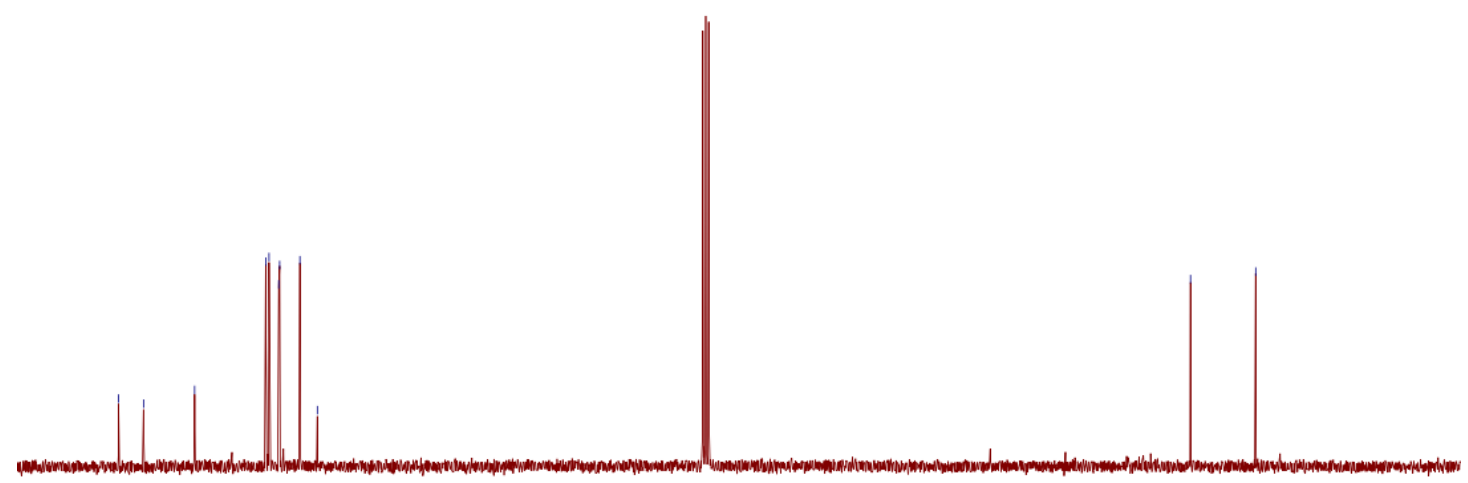

$\frac{1}{150}$

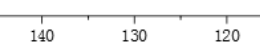

$80 \underset{\mathrm{f} 1(\mathrm{ppm})}{70}$ 
${ }^{1} \mathrm{H}$ NMR (400 MHz, $\mathrm{CDCl}_{3}$ ) of $\mathbf{5 s}$

무요요

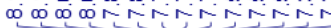

लొ

लंगुल लंख
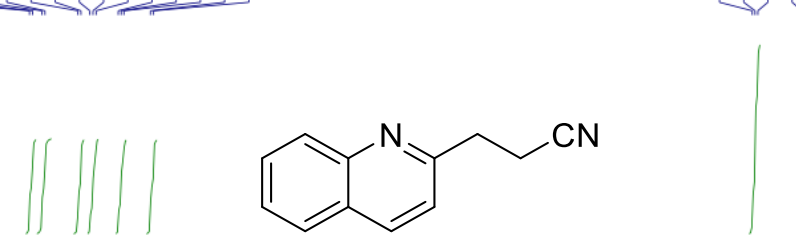<smiles>N#CCCc1ccc2ccccc2n1</smiles>

$\mathrm{CN}$ 
${ }^{1} \mathrm{H}$ NMR (400 MHz, $\mathrm{CDCl}_{3}$ ) of $\mathbf{5 t}$

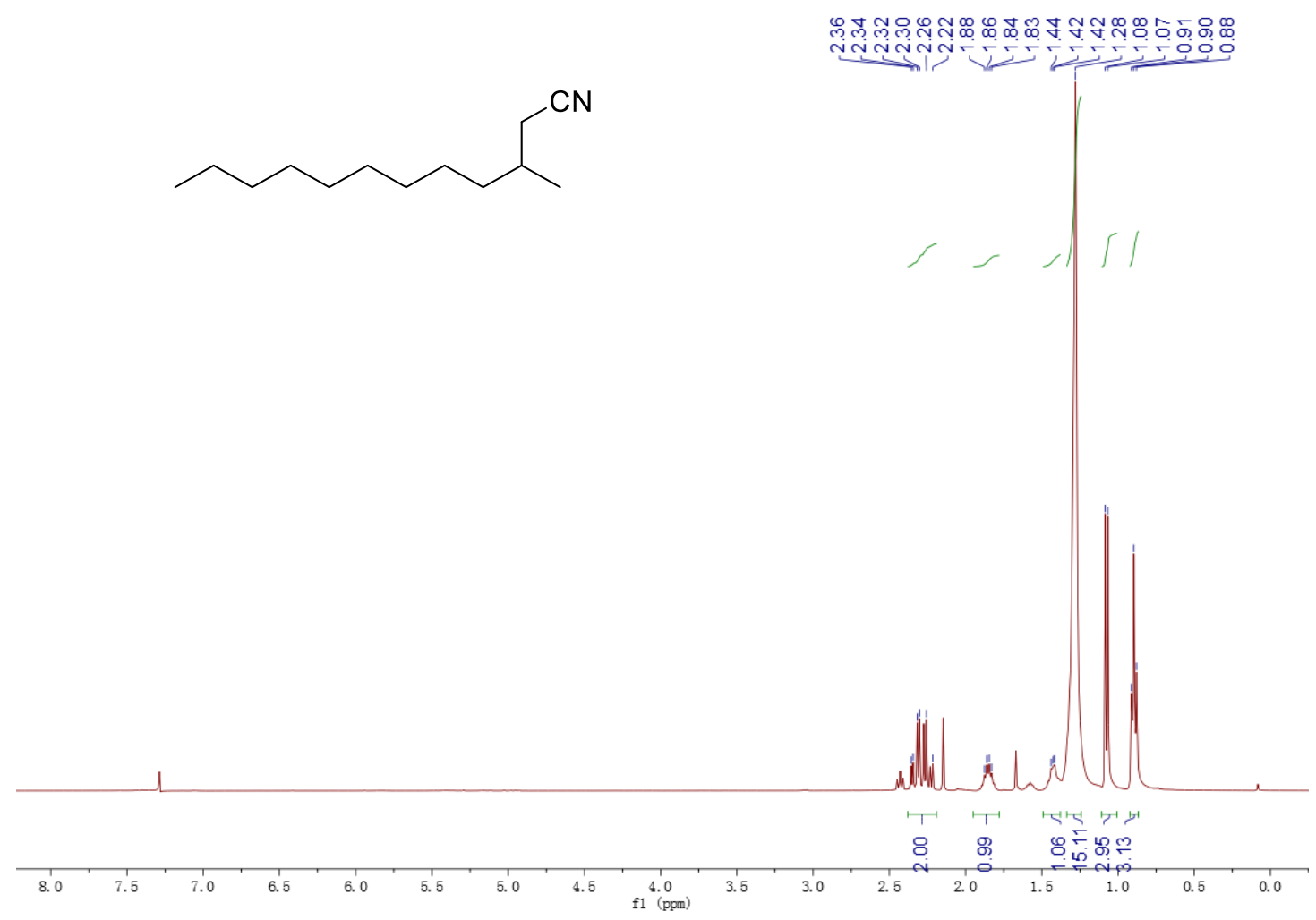

${ }^{13} \mathrm{C}\{1 \mathrm{H}\} \mathrm{NMR}\left(100 \mathrm{MHz}, \mathrm{CDCl}_{3}\right)$ of $\mathbf{5 t}$
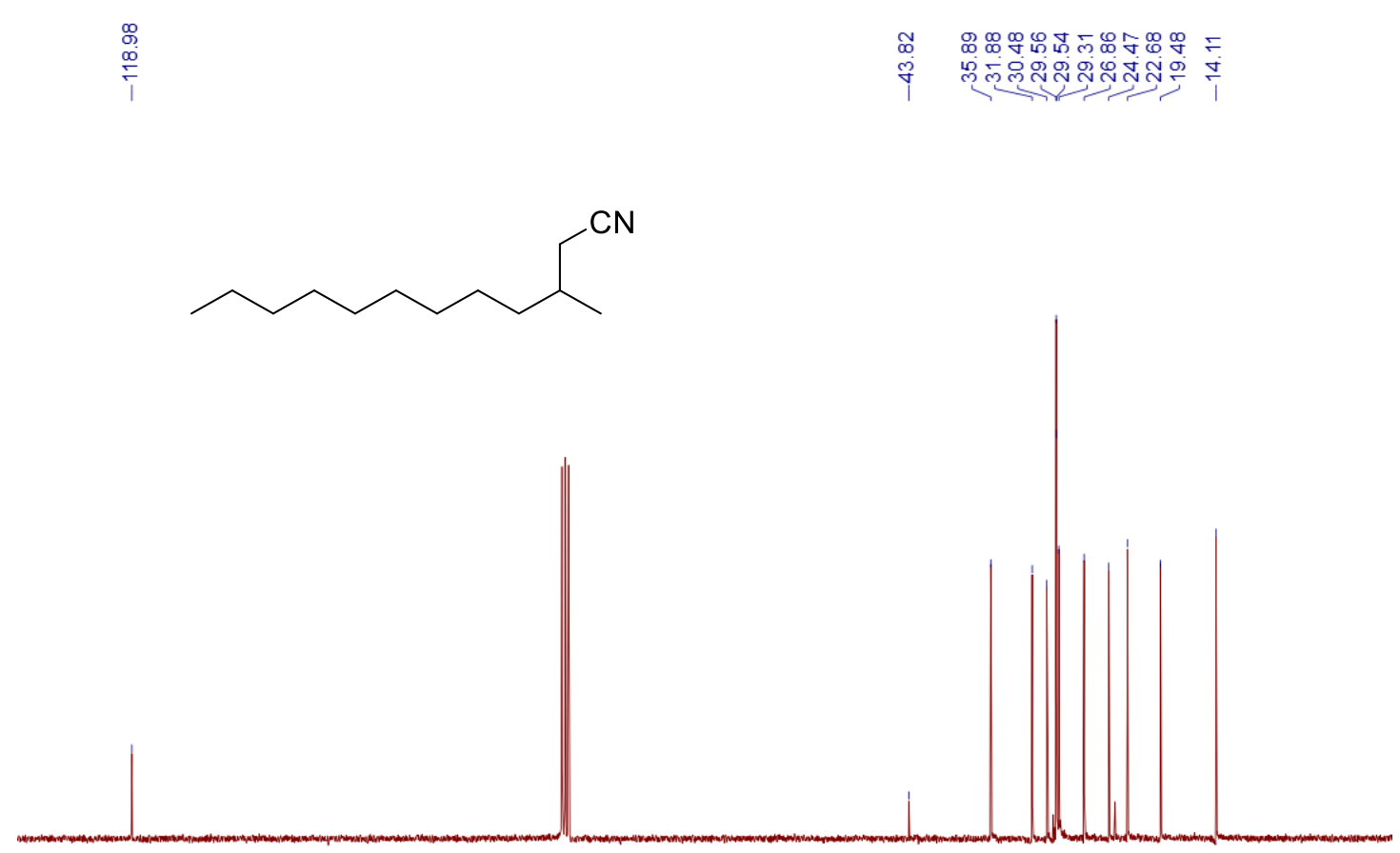

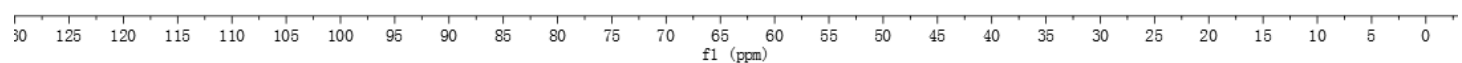


${ }^{1} \mathrm{H}$ NMR (400 MHz, $\left.\mathrm{CDCl}_{3}\right)$ of $\mathbf{5 u}$

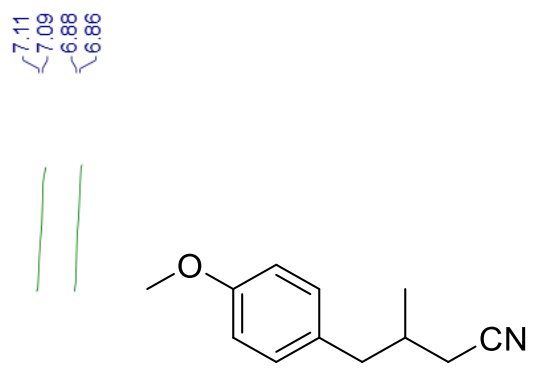

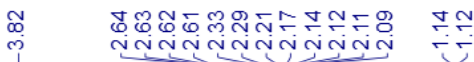

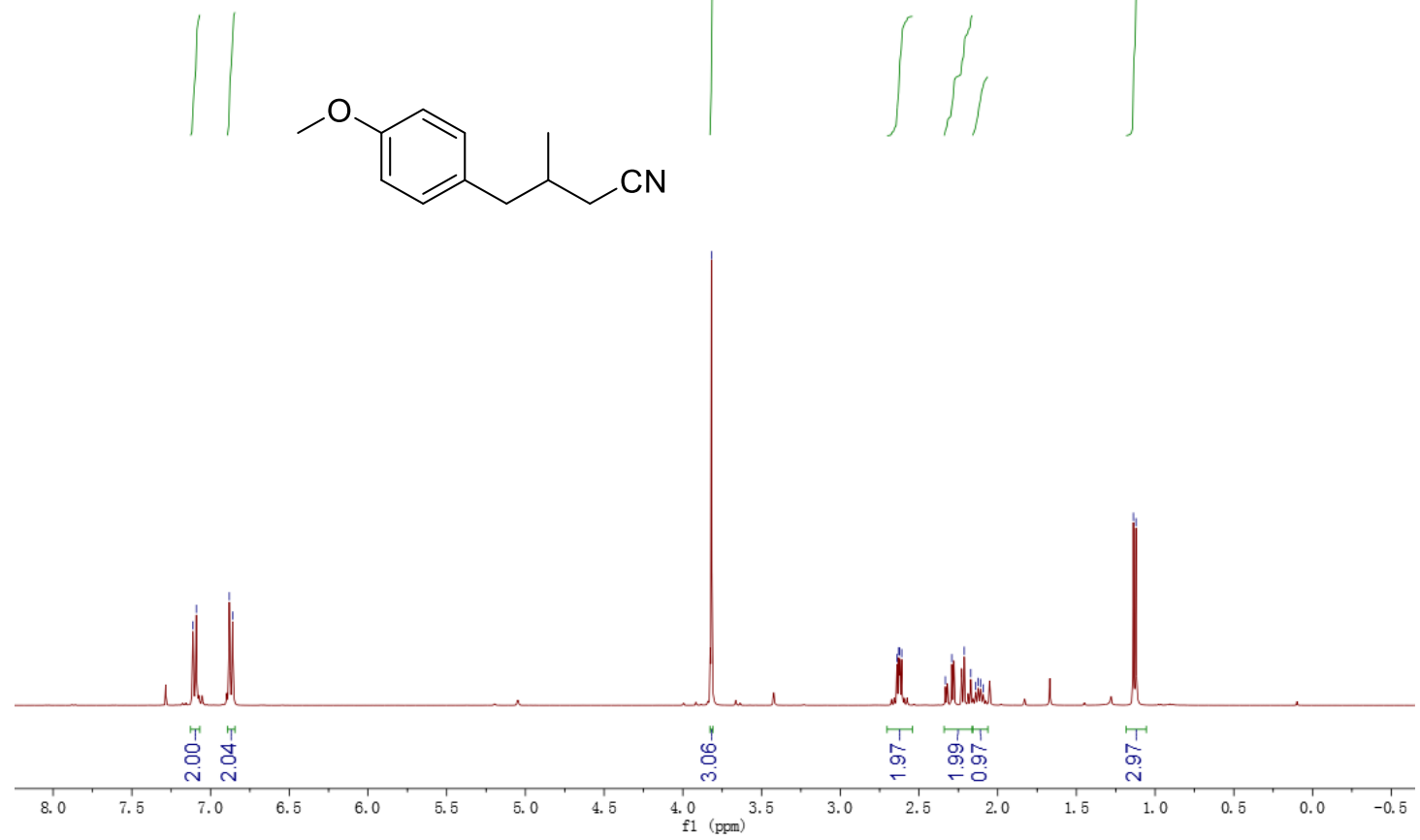

${ }^{13} \mathrm{C}\{1 \mathrm{H}\} \mathrm{NMR}\left(100 \mathrm{MHz}, \mathrm{CDCl}_{3}\right)$ of $\mathbf{5 u}$

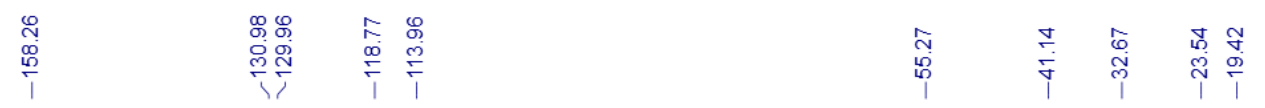<smiles>COc1ccc(CC(C)CC#N)cc1</smiles>

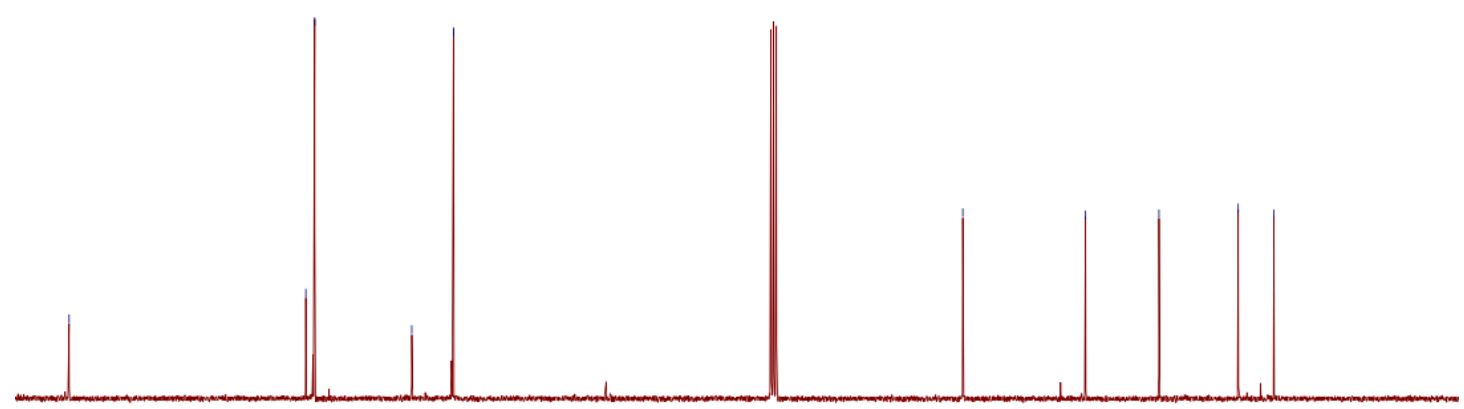

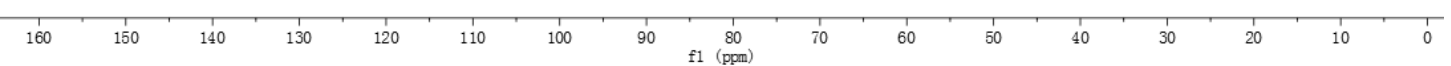


${ }^{1} \mathrm{H}$ NMR (400 MHz, $\left.\mathrm{CDCl}_{3}\right)$ of $\mathbf{5 v}$

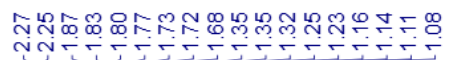<smiles>N#CCC1CCCCC1</smiles>
ה⿻一亅八.
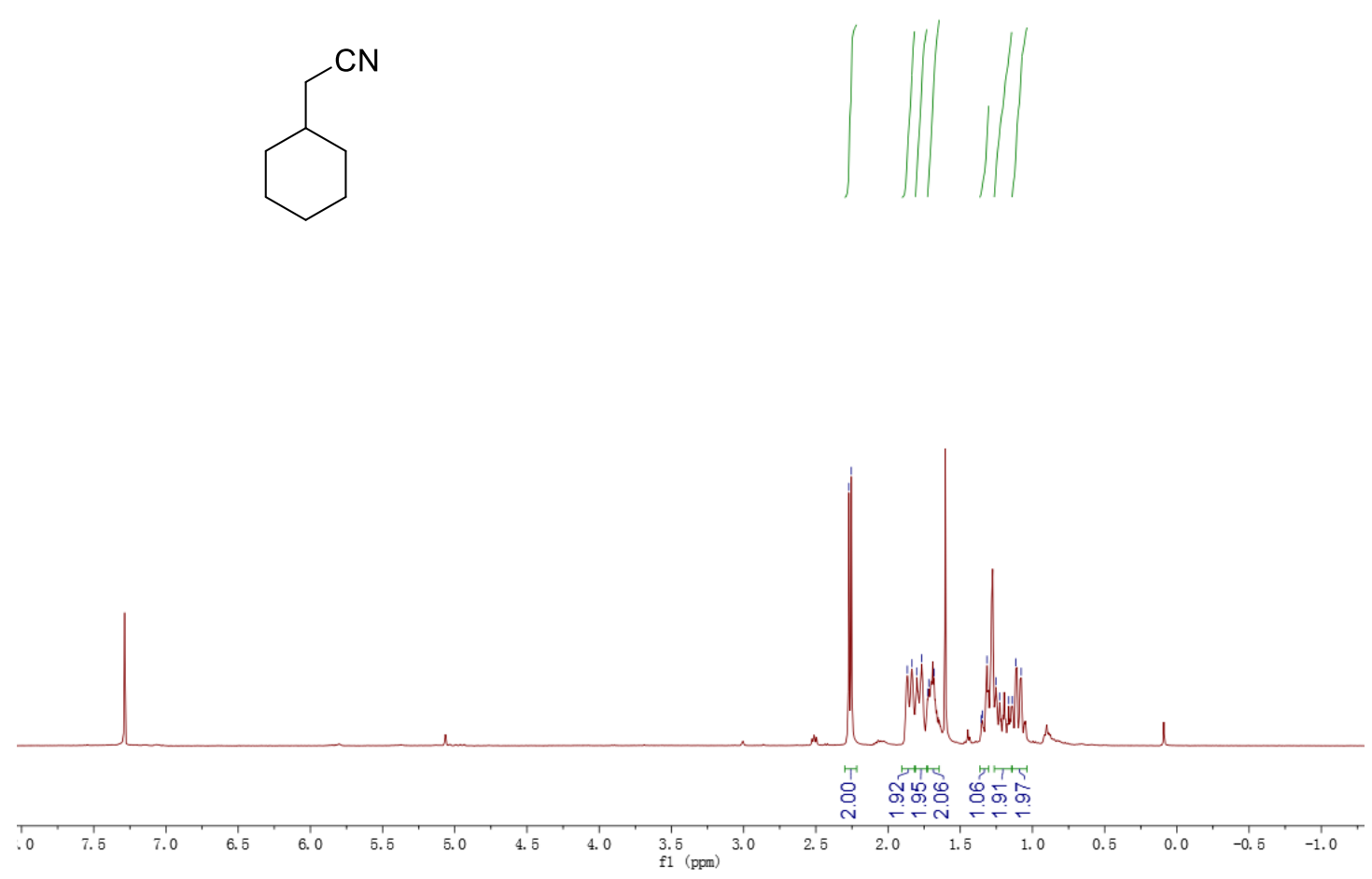

${ }^{13} \mathrm{C}\{1 \mathrm{H}\} \mathrm{NMR}\left(100 \mathrm{MHz}, \mathrm{CDCl}_{3}\right)$ of $\mathbf{5 v}$

$\infty$
$\infty$
$\infty$
$\stackrel{\infty}{\sigma}$

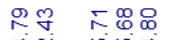

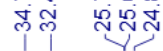

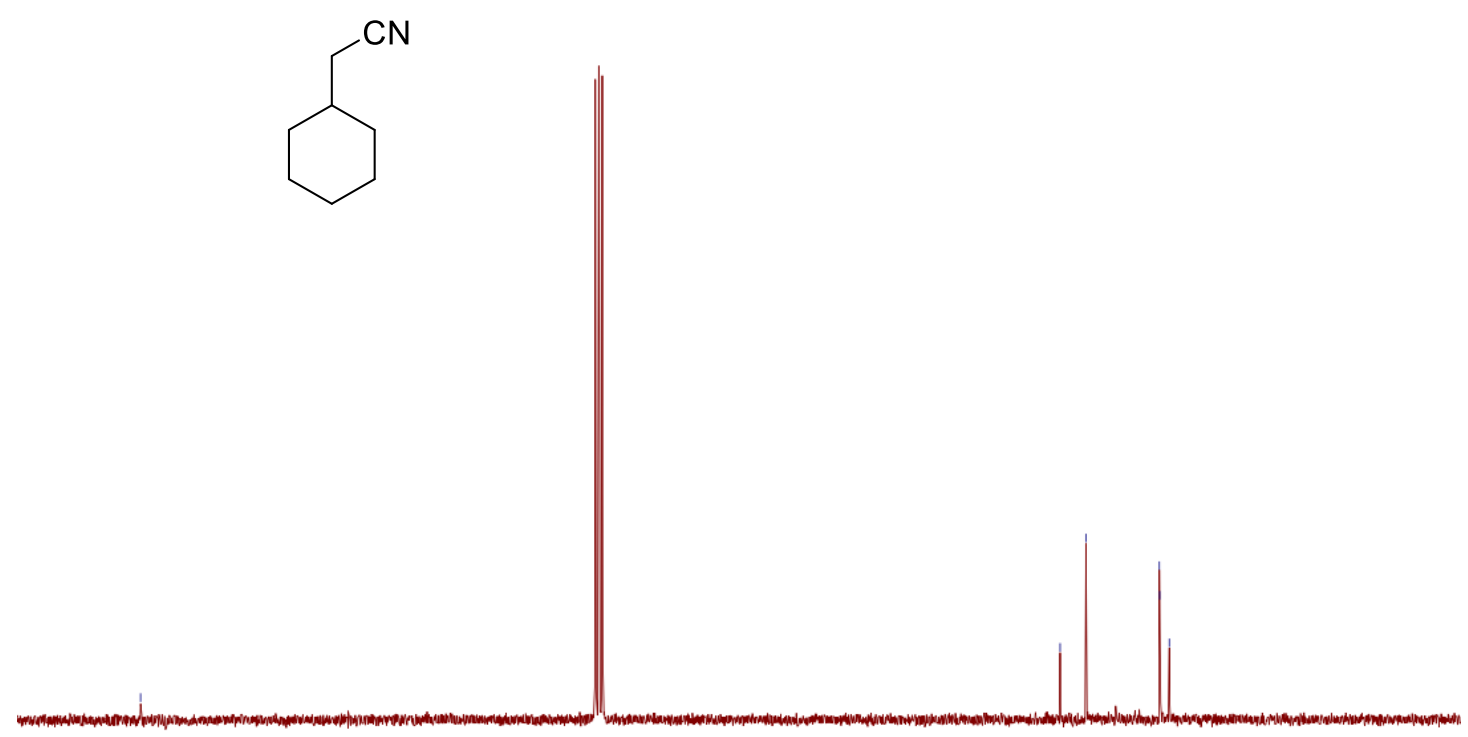

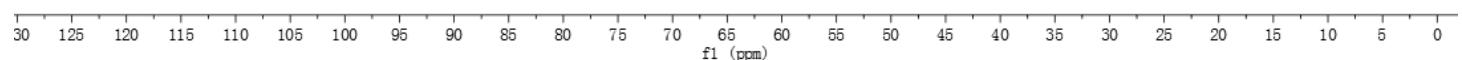


${ }^{1} \mathrm{H}$ NMR (400 MHz, $\left.\mathrm{CDCl}_{3}\right)$ of $\mathbf{5 w}$

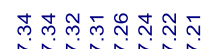

inition

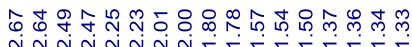
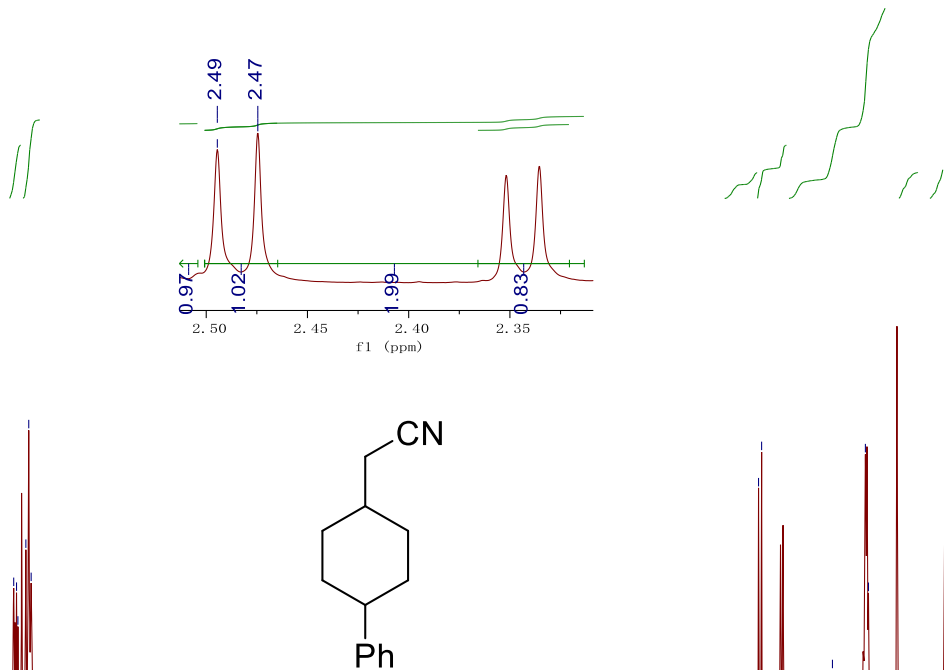

$\mathrm{Ph}$
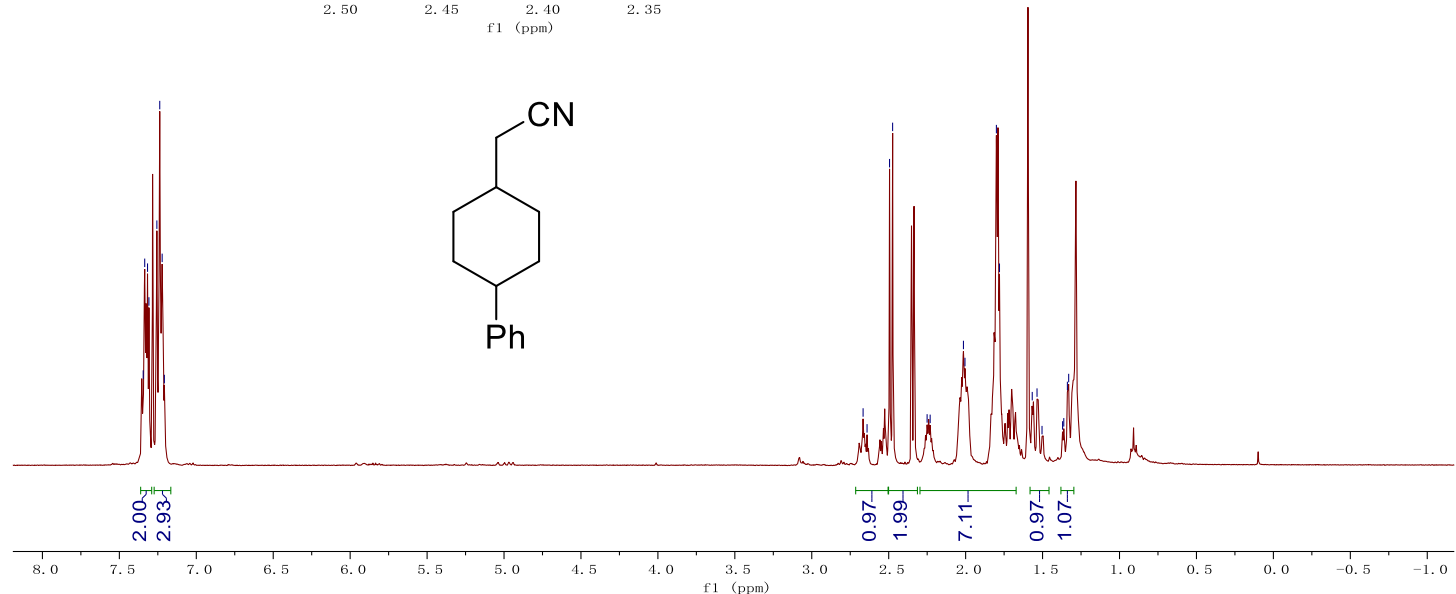

${ }^{13} \mathrm{C}\{1 \mathrm{H}\}$ NMR $\left(100 \mathrm{MHz}, \mathrm{CDCl}_{3}\right)$ of $\mathbf{5 w}$

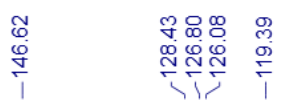

每<smiles>N#CCC1CCC(c2ccccc2)CC1</smiles>

$\mathrm{Ph}$
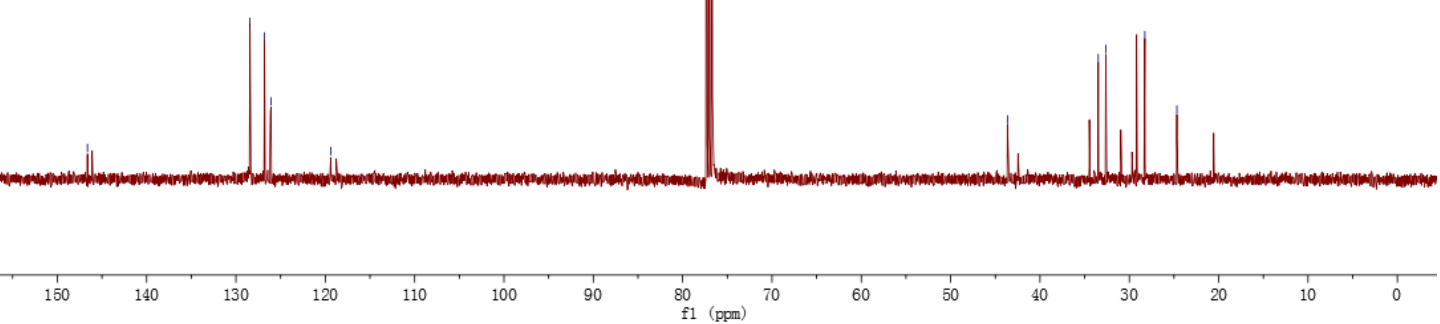
${ }^{1} \mathrm{H}$ NMR (400 MHz, $\left.\mathrm{CDCl}_{3}\right)$ of $\mathbf{5 x}$

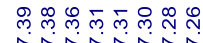

rintan

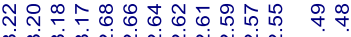

m m mं m v

$\|$<smiles>CC(CC#N)c1ccccc1</smiles>
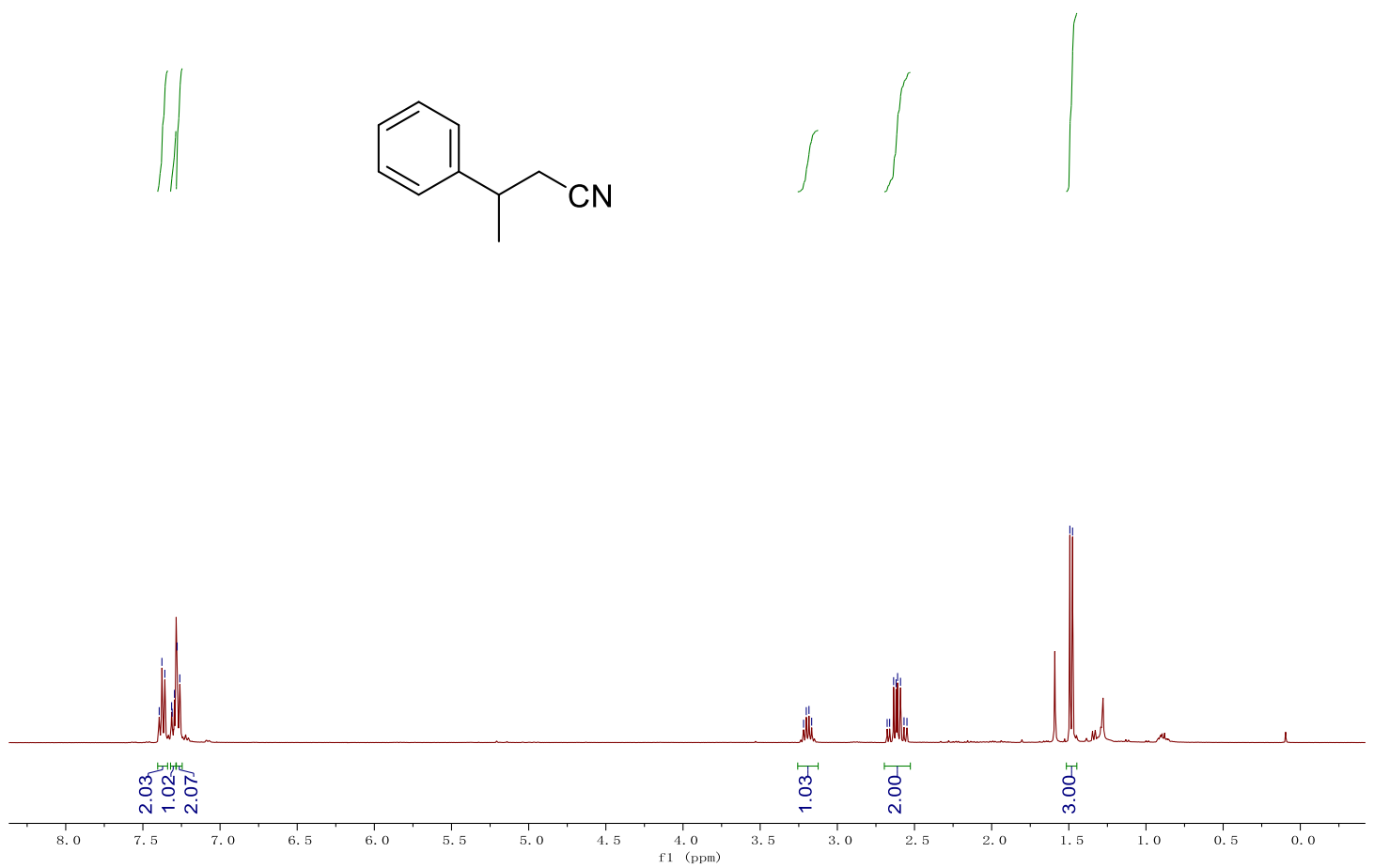

${ }^{13} \mathrm{C}\{1 \mathrm{H}\} \mathrm{NMR}\left(100 \mathrm{MHz}, \mathrm{CDCl}_{3}\right)$ of $\mathbf{5} \mathbf{x}$

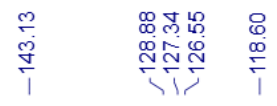

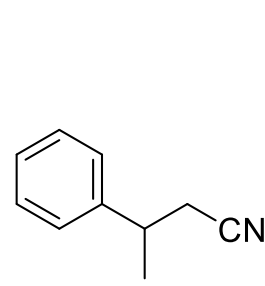

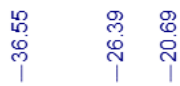
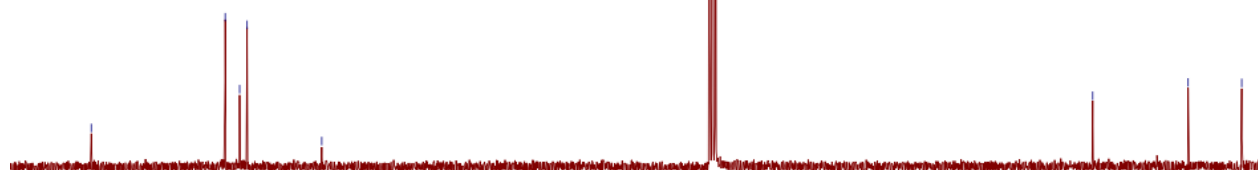

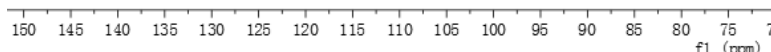


${ }^{1} \mathrm{H}$ NMR $\left(400 \mathrm{MHz}, \mathrm{CDCl}_{3}\right)$ of $\mathbf{5 y}$

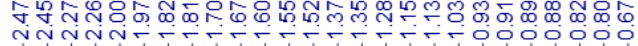
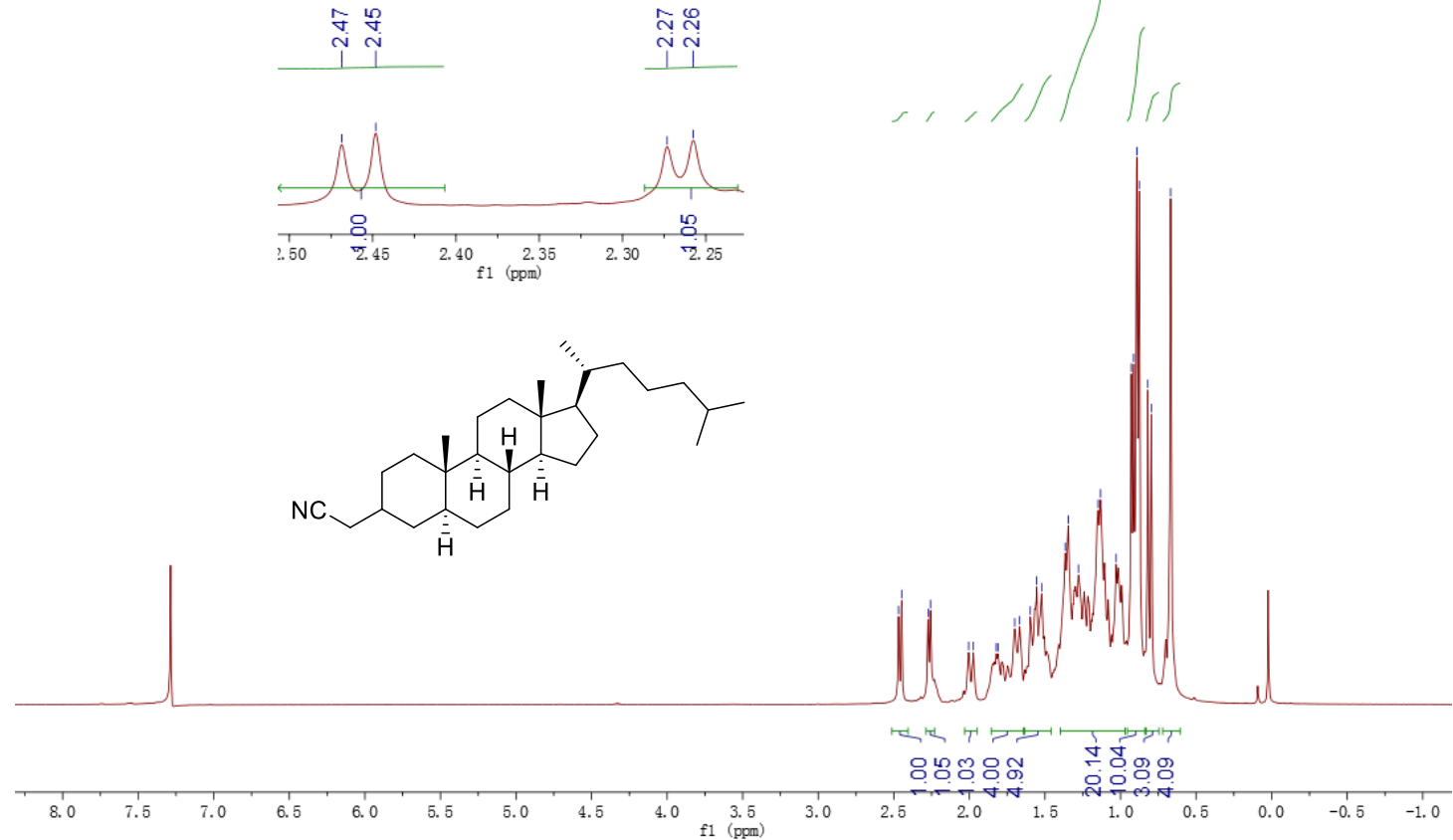

${ }^{13} \mathrm{C}\{1 \mathrm{H}\} \mathrm{NMR}\left(100 \mathrm{MHz}, \mathrm{CDCl}_{3}\right)$ of $\mathbf{5 y}$

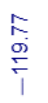

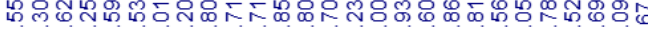

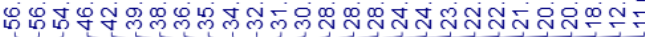<smiles>CC(C)CCC[C@H](C)C1CCC2C3CC[C@H]4CC(CC#N)CCC4(C)C3CCC21C</smiles>

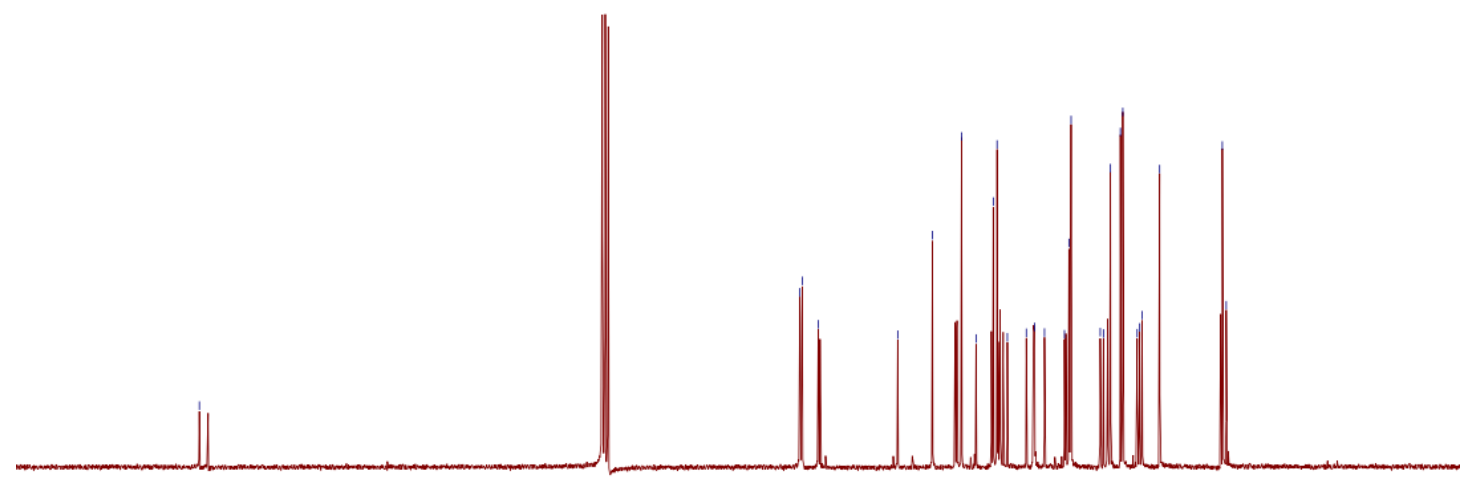

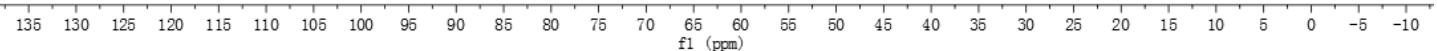

